University of Rhode Island

DigitalCommons@URI

Open Access Master's Theses

2007

\title{
AIR-SEA SCALAR FLUX DUE TO WIND-GENERATED NEAR- SURFACE TURBULENT EDDIES: THE EDDY RENEWAL MODEL
}

Eric G. Van Inwegen

University of Rhode Island, eric.vaninwegen@gmail.com

Follow this and additional works at: https://digitalcommons.uri.edu/theses

\section{Recommended Citation}

Van Inwegen, Eric G., "AIR-SEA SCALAR FLUX DUE TO WIND-GENERATED NEAR-SURFACE TURBULENT EDDIES: THE EDDY RENEWAL MODEL" (2007). Open Access Master's Theses. Paper 1484.

https://digitalcommons.uri.edu/theses/1484

This Thesis is brought to you for free and open access by DigitalCommons@URI. It has been accepted for inclusion in Open Access Master's Theses by an authorized administrator of DigitalCommons@URI. For more information, please contact digitalcommons-group@uri.edu. 


\title{
AIR-SEA SCALAR FLUX DUE TO WIND-GENERATED NEAR-SURFACE TURBULENT EDDIES: THE EDDY RENEWAL MODEL
}

\author{
By
}

Eric G. VanInwegen

A thesis submitted in partial fulfillment of the requirements for the degree of Master of Science

in

Oceanography

University of Rhode Island 


\section{Master of Science Thesis \\ of}

Eric G. VanInwegen

\section{APPROVED:}

Thesis Committee:

\section{Major Professor}

Dean of the Graduate School

University of Rhode Island 


\section{Abstract}

Based on analyses of infrared images of the air-water interface, a new model of surface tracer flux is presented (eddy renewal model). In contrast to the currently used model (surface renewal model), which assumes that water motions are driven solely by breaking event-like conditions (or sudden bursts of turbulence), the new model posits that water motions are driven by Langmuir-like turbulent eddies (or more steady-state conditions). These wind-generated turbulent eddies arrange to create elongated warm patches of upwelled water between long streaks of colder downwelling water. In analyzing the images taken during GasEx2001 expedition in 2001 in the Equatorial Pacific waters and laboratory experiments in the AEOLOTRON wind wave tank at University of Heidelberg in October 2004, both breaking event-like motions and Langmuir-like eddies are present, and thus the new model complements the old (as opposed to replacing it). Analysis of the bulk temperature estimates from the new model show that they are quite similar to those from the old model, and, perhaps, hint at conditions where one model may be more appropriate than the other. 


\section{Acknowledgement}

This work would not have been possible without the invaluable advice, input, and assistance of many capable people. The data reported in this work has all come from other sources. The majority of the data used in the results section comes from the GasEx2001 expedition collected onboard the NOAA $\mathrm{R} / \mathrm{V}$ Ronald H. Brown during the 2001 experiments in the Southern Equatorial Pacific. The wind-wave tank data comes from the AEOLOTRON group at the University of Heidelberg, in Heidelberg, German, and was collected in 2004 by the staff at that facility. The Co-OP data was collected during the work in the Northern Atlantic in 1997. Christoph Garbe in the AEOLOTRON group was instrumental in his efforts make the AEOLOTRON and Co-OP data available. The MatLab code used to generate the model eddy was previously created at the University of Rhode Island's Graduate School of Oceanography (by John Wendelbo and Tetsu Hara). Funding for this study was provided by the NOAA Global Carbon Cycle (GCC) Program.

Lastly, I would like to thank my family for their constant love and devotion, my friends and colleagues for their unwavering support, my committee for their astute input, and my advisor Tetsu Hara for his constant, insightful, and, most importantly, patient guidance. 
$\underline{\text { Table of Contents }}$

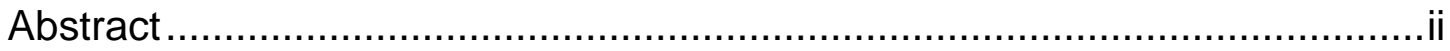

Acknowledgement ................................................................................

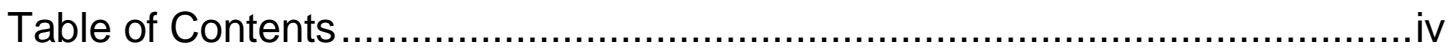

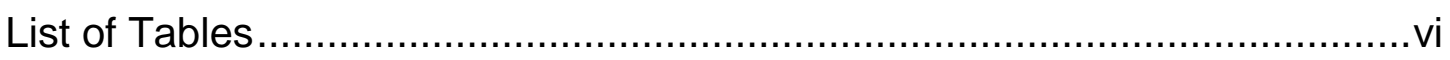

List of Figures …….......................................................................... vii

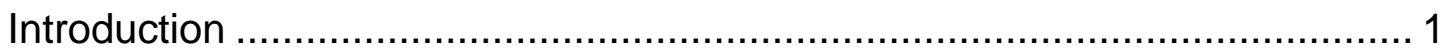

Chapter 1. Background Physics and Surface Renewal Model

Section 1. Surface Renewal Model - Physics of Cooling .................... 4

Section 2. Surface Renewal Model - Statistical Distribution ............... 9

Section 3. Gas Flux Derived from Heat Flux .................................. 12

Section 4. Limitations of the Surface Renewal Model....................... 14

Chapter 2. The Eddy Renewal Model

Section 1. Eddy Renewal Model - Upwelling Solution ...................... 16

Section 2. Eddy Renewal Model - Eddy Interior Solution................... 21

Section 3. Normalization and Solution of the Temperature and

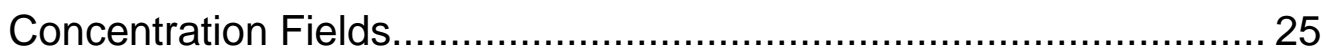

Chapter 3. Comparison of Models

Section 1. Comparison of Model Characteristics ............................. 30

Section 2. Single Renewal Event / Eddy Comparison ....................... 31

Section 3. Comparison of Model Parameters ................................... 35

Section 4. Comparison of Identical Parameter Distributions............... 37 
Chapter 4. Applying the ERM to Infrared Sea-Surface Images

Section 1. Methodology of Image Analysis ...................................... 39

Section 2. Results of Image Analysis ............................................ 46

Section 3. Discussion of Image Analysis Results ............................ 55

Section 4. Statistics Obtained From Image Analysis Results ............. 61

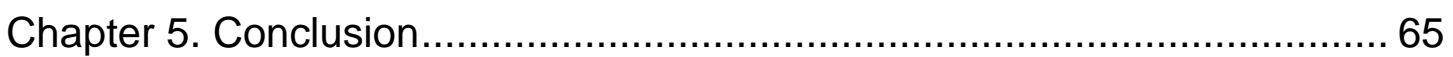

Appendix A. Instrument Specifications and Experiment Details .................. 67

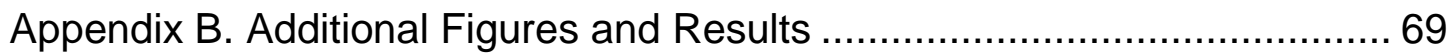

Appendix C. Computational Methods and Algorithms ................................ 85

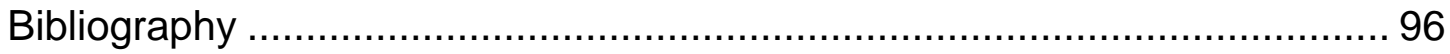




\section{List of Tables}

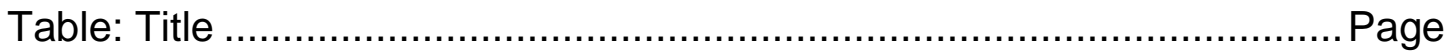

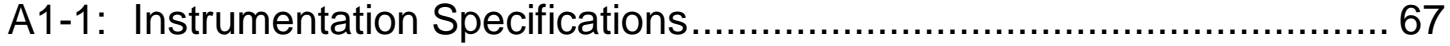

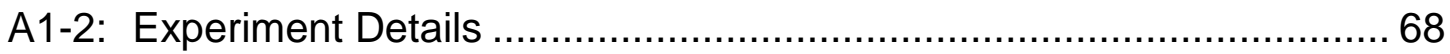




\section{List of Figures}

Figure: Caption Page

1: $\quad$ Two models of profile concentration change over time..................... 8

2: $\quad$ Surface temperature renewal plots ........................................ 9

3: $\quad$ Temperature distributions; hypothetical SRM and observed ............ 11

4: $\quad$ A representative infrared sea-surface image ................................ 16

5: $\quad$ A schematic cross-section of surface eddies............................. 17

6: $\quad$ The non-dimensional solutions to the ERM. ............................. 27

7: $\quad$ Comparison of surface temperatures curves for similar regions of SRM and ERM, with matching mean temperatures........................... 31

8: Histograms of two representative regions, comparing the differences in cooling between the two models ...................................... 32

9: Representational volumes of water acted upon by the two different

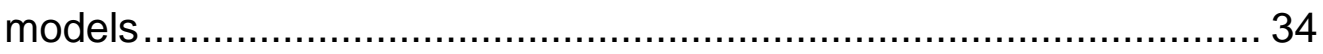

10: Comparison of Temperature Distributions .............................. 38

11: $\quad$ Finding the local maxima \& minima ............................................ 39

12: Comparison of results for two different bulk temperature estimation methods.

13: Model fit to one temperature line, showing observed data, modeled temperature curves, and Bulk Temperature estimations

14: The results of preprocessing on an image with dead pixels and high levels of pixilation 
16: Image analysis of a breaking event

17: Image analysis after a breaking wave has lost much of its energy to

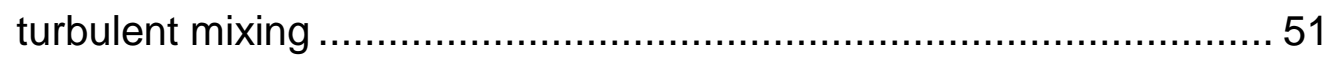

18: Image analysis with higher levels of reflected infrared levels ............ 52

19: Image analysis of streaky patterns in a wind-wave tank.................... 53

20: Image analysis of a frame from a wind-wave tank showing patterns more like those from in situ images ............................................. 54

21: Non-dimensionalized eddy temperature curves compared to ERM

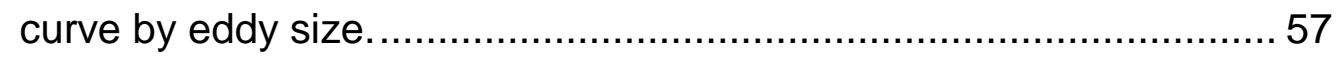

22: Percent of Eddies with an Error Squared Per Pixel value smaller than

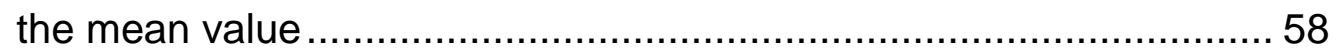

23: Average, non-dimensionalized eddy curves, using only the eddies with an error squared per pixel smaller than the mean values 59

24: Correlation charts for eddy size vs. intensity and size vs. bulk

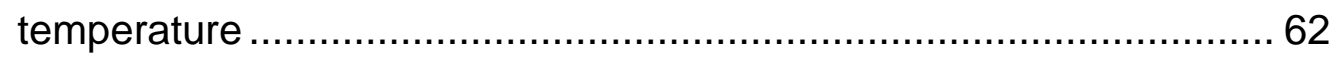

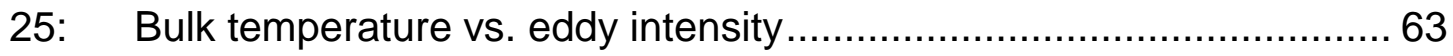

26: Histograms of parameters from image analysis .............................. 64

27: Distribution of surface divergence maximum velocities ..................... 64

Figures in the Apendicies:

A-01: Time series plot of bulk temperature estimates from two models (for file

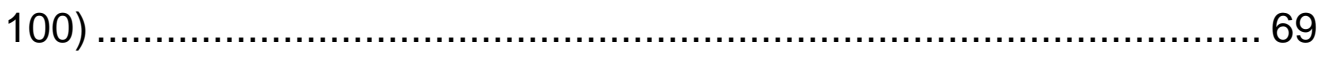

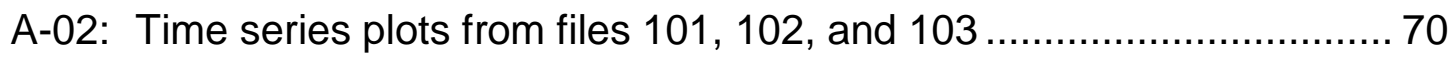




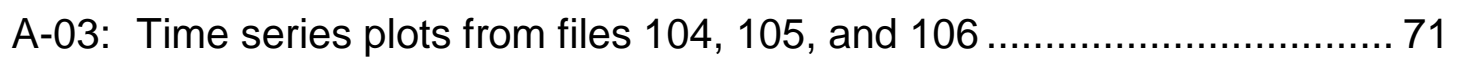

A-04: Time series plots from files 107,108 , and 109 ............................. 72

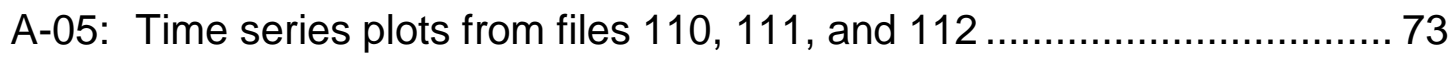

A-06: Time series plots from Aeolotron file 001 ...................................... 74

A-07: Time series plots from Aeolotron files 002, 003, and 004 ................. 75

A-08: Time series plots from Aeolotron files 005 and 011 ......................... 76

A-09: Time series plots from Aeolotron files 012, 013, and 014 ................ 77

A-10: Time series plots from Aeolotron files 015, 016, and 017................ 78

A-11: Time series plots from Aeolotron files 018, 019, and 020 ................. 79

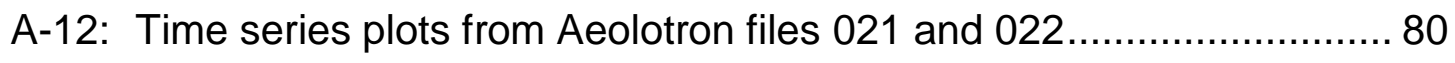

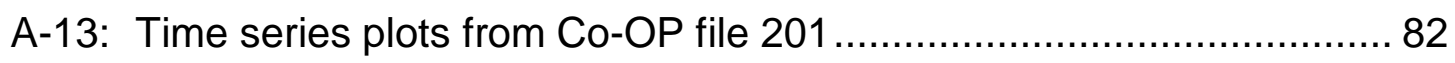

A-14: Time series plots from Co-OP file 202 ......................................... 83

A-15: Time series plots from Co-OP file 203 …….................................. 84 
Introduction

In any air-sea climatological model, the importance of the transfer of heat or gases (especially greenhouse gases such as $\mathrm{CO}_{2}$ ) between water and atmosphere cannot be understated. However, the physical processes that control such transfers are only recently being understood. Compounding the issue is that the transfers are affected by many variables, including, but not limited to, wave action, wind speed, temperatures of atmosphere and seas, concentrations of gases, coefficients of molecular diffusivity, and condition of surface waters (e.g. presence of surfactants). (Frew, et. al., 2004). Within the last few decades, strides have been made in understanding this complex system. Aside from understanding the individual variables, much research has been conducted to clarify how those variables interact with each other and how to best measure the transfer (Jähne and Haußecker, 1998).

Given the range of values that have been calculated through indirect and direct gas measurement techniques (McGillis, et. al., 2001), methods have been invented to use heat as a proxy tracer. One of the problems with direct gas transfer measurements is that it is extremely difficult to detect the concentration fluctuations or gradients, due to the physical limitations of in situ gas concentration measurements. Methods using tracers and patches heated with short-bursts of an infrared laser have been used to calculate transfer velocities (e.g. McGillis, et. al., 2001), however their efficacy has 
been questioned (Jacobs, et. al., 2002). The use of infra-red imaging allows very accurate measurements of the water surface temperature to be collected with minimal impact on the system (Haußecker, Reinelt, and Jähne, 1995). If the heat transfer velocities are determined to high levels of accuracy using the infrared measurements, the gas transfer velocities can be estimated using the Schmidt number scaling and a small number of non-invasive gas concentration measurements (Frew, et. al., 2004).

In order to interpret observed surface temperature variation and to estimate the heat transfer velocity, a mechanical model of "surface renewal" has been used. In this model, it is assumed that there remains a boundary "thin-film" at the surface through which tracers must pass. This boundary is a result of the stronger effects of viscosity near the surface and can be visualized in experiments with reactant dyes and tracers, or by measuring the temperature profile and surface temperature field. The Surface Renewal Model assumes that this thin film is periodically renewed by bursts of turbulent eddies, which refresh the waters in the surface boundary layer. The transfer of the gas is determined by the frequency of these renewal events, which is related to the wind speed at ten meters. This model can be used to explain the observed surface temperature distribution in a statistical sense (Garbe, 2001). With finer scale measurements, however, it appears that this model may not be mechanistically correct.

The main objective of this study is to develop a new model of the near surface turbulence, which is more consistent with the observed small scale 
surface temperature patterns. Models of gas transfer being effected by eddies have been presented in the past (e.g. Fortescue and Pearson in 1967, Lamont and Scott in 1970, Csanady in 1990, and Atmane, Asher and Jessup in 2004), however none have developed a model based on steady-state assumptions (i.e. based on the apparent longevity of observed surface temperature patterns) or attempted to match a modeled temperature curve to infrared images of individual eddies, as is presented here. The model is applied to both field and laboratory data of infrared observations. The results are then compared with those based on the existing surface renewal model, in order to examine the validity of the new model. 


\section{Chapter 1. Background Physics and Surface Renewal Model}

\section{Section 1. Surface Renewal Model - Physics of Cooling}

Although the temperature of the air can be warmer than that of the water, there usually exists a "cool-skin" of one millimeter or less at the interface (Soloviev and Schlüssel, 1994). This skin is a result of the latent heat transfer from water to air that persists, except in certain circumstances (e.g. fog). When environmental conditions are not changing rapidly, one can assume that there is a layer below the sea surface where the heat flux is constant with depth (that is, there is no significant heating or cooling within this layer). Also, it is apparent that turbulent flux is responsible for heat flux away from the boundary, and molecular diffusion is the primary mechanism only in a thin diffusive sublayer close to the boundary (Frew, et. al., 2004). In order to model the heat transfer process across the diffusive layer, one must, of course, start with the basic governing equations. The heat flux is proportional to the temperature gradient, as in

$$
j_{H}=-k \nabla T,
$$

where, $j_{H}$ is heat flux, $T$ is temperature, and $k$ is thermal conductivity or

$$
\kappa=k / \rho C_{P},
$$

where $K$ is thermal diffusivity, $\rho$ is density and $C_{P}$ is specific heat. The conservation of heat can be expressed as

$$
\frac{d T}{d t}=\frac{-1}{\left(C_{p} \rho\right)} \nabla j_{H}
$$


We can then combine equations [1.1.1] and [1.1.3] to obtain

$$
\frac{d T}{d t}=\kappa \Delta T
$$

where

$$
\Delta=\frac{\partial^{2}}{\partial x^{2}}+\frac{\partial^{2}}{\partial y^{2}}+\frac{\partial^{2}}{\partial z^{2}} .
$$

Expanding [1.1.4] yields

$$
\frac{\partial T}{\partial t}+u \frac{\partial T}{\partial x}+v \frac{\partial T}{\partial y}+w \frac{\partial T}{\partial z}=\kappa\left(\frac{\partial^{2} T}{\partial x^{2}}+\frac{\partial^{2} T}{\partial y^{2}}+\frac{\partial^{2} T}{\partial z^{2}}\right)
$$

Solving [1.1.6] requires some understanding of the boundary conditions. One assumption of all of the heat transfer models is that the temperature at $z=-\infty$ is uniform; this is called the "bulk temperature" ( $\left.T_{B}\right)$. That is, at some distance below the surface, the turbulent mixing is sufficiently strong and the temperature approaches a constant. Given the definition of the "well-mixed layer," this is a good approximation for the temperature at a depth well below the surface, but still well above the thermocline. A constant bulk concentration is a good approximation for gasses, as well.

The surface boundary condition, however, differs between gasses and heat. In order to solve [1.1.6], at the surface, either the temperature (or gas concentration) or the flux must be specified. For heat, a constant flux is the more appropriate choice, because the net air-sea heat flux is mainly controlled by the air-side turbulence. For insoluble to slightly soluble gasses, however, a constant surface concentration is a better approximation, due to 
the higher turbulence in the air near the water surface keeping the concentration of the gasses well mixed.

In principle, the temperature field can be obtained by solving [1.1.6] provided the water motion $(u, v, w)$ is known. In reality, the velocity field due to near surface turbulent eddies is not explicitly known, and hence some assumptions must be made.

The Surface Renewal Model (as presented by Garbe in 2001) assumes that the diffusive sublayer is periodically renewed; that is, the waters in the surface boundary layer are periodically refreshed with bulk temperature waters, and thus the temperature is periodically equal to the bulk temperature. In between the renewal events, it is assumed that 1.) advection plays no role in heat diffusion and 2.) the gradients are zero in the horizontal. Thus the equation [1.1.6] reduces to

$$
\frac{\partial T}{\partial t}=\kappa\left(\frac{\partial^{2} T}{\partial z^{2}}\right)
$$

Using the same reasoning and assumptions, one can derive a similar equation for the diffusion of a sparingly soluble gas tracer. Thus,

$$
\frac{\partial C}{\partial t}=D\left(\frac{\partial^{2} C}{\partial z^{2}}\right)
$$

where $C$ is the concentration of the gas, and $D$ is the diffusivity constant.

For a gas concentration problem, we set the boundary conditions as

$$
\begin{array}{lll}
C=C_{S} & \text { at } & z=0 \\
C=C_{B} & \text { at } & z=-\infty
\end{array}
$$

The solution for the gas equation is then 


$$
C(z, t)=\left(C_{S}-C_{B}\right) \operatorname{erfC}\left(\frac{-z}{2 \sqrt{D t}}\right)+C_{B}
$$

where $C_{B}$ is the concentration at depth, $C_{S}$ is the surface concentration (assumed to be constant) and the complimentary error function, $\operatorname{erfc}(u)$, is defined by

$$
\operatorname{erf}(u) \equiv\left(\frac{2}{\sqrt{\pi}}\right) \int_{0}^{u} e^{-\eta^{2}} d \eta
$$

and

$$
\operatorname{erfc}(u) \equiv 1-\operatorname{erf}(u)
$$

The surface flux is described by

$$
j_{G, z=0}=\left.D \frac{\partial C}{\partial z}\right|_{z=0}=\frac{\sqrt{D}\left(C_{S}-C_{B}\right)}{\sqrt{\pi t}} .
$$

Therefore, the concentration flux varies with time.

For the temperature, the surface flux (jo) remains constant, while the surface temperature varies with time. Since the heat flux $j_{H}$ satisfies the same diffusion equation as $T$, the solution is basically identical to that of $C$, i.e.,

$$
j_{H}(z, t)=j_{0} \operatorname{erfc}\left(\frac{-z}{2 \sqrt{\kappa t}}\right) .
$$

To solve for the temperature, equation [1.1.1] is combined with [1.1.15] and the integral is taken, giving

$$
T(z, t)=\frac{-j_{0}(2 \sqrt{\kappa t})}{k}\left[\frac{-z}{2 \sqrt{\kappa t}}\left(\operatorname{erfc}\left(\frac{-z}{2 \sqrt{\kappa t}}\right)\right)-\frac{e^{-\left(\frac{-z}{2 \sqrt{\kappa t}}\right)^{2}}}{\sqrt{\pi}}\right]+T_{B} .
$$

At $z=0$, equation [1.1.16] reduces to 


$$
T(0, t)=\frac{-j_{0} 2 \sqrt{t}}{C_{p} \rho \sqrt{\pi \kappa}}+T_{B} .
$$

The difference between a constant surface concentration (gas) and a constant surface flux (heat) gives two different solutions over time (Figure 1). In the first case, the surface concentration remains one fixed value; however the flux changes with time. In the second case, the surface temperature changes according to equation [1.1.17], while the flux remains constant. This disparity will be discussed in later sections.

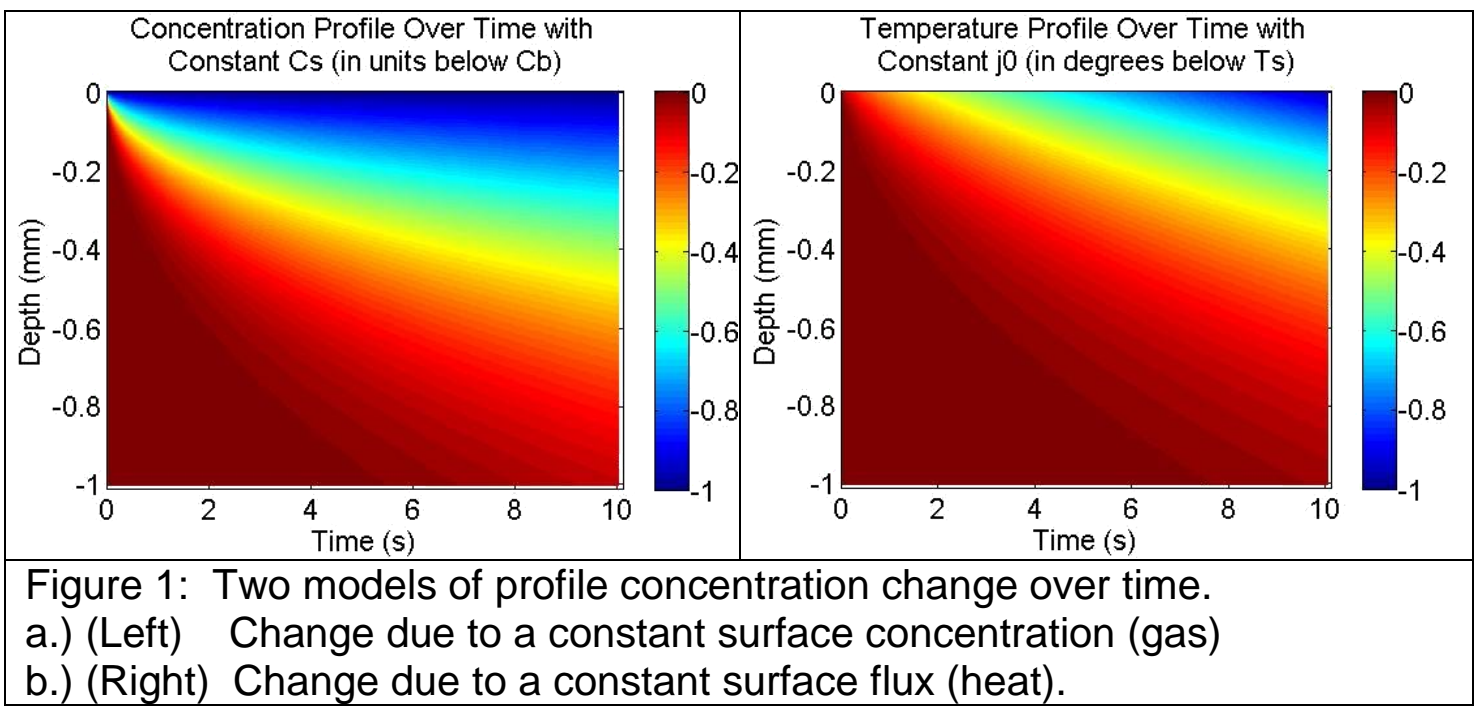




\section{Section 2. Surface Renewal Model - Statistical Distribution}

In the Surface Renewal Model, the temperature of a parcel of surface of sea water is described by equation [1.1.17] until the surface renewal event occurs and "resets" the temperature of the water column to $T_{B}$ and the cooling begins again. This event is considered to occur instantaneously. This periodic renewal is assumed to be a random process, and thus treated statistically.

If a surface patch renews at a set time interval $\tau$, then the temperature for that parcel will resemble that of Figure 2a. However, it is more correct to imagine that the interval between the renewal events varies randomly. Then, the temperature observed for the parcel will more likely resemble Figure $2 b$. It is important to note that, in viewing the sea surface infrared images, the temperature varies spatially. Therefore, the surface renewal model is not strictly valid. If,
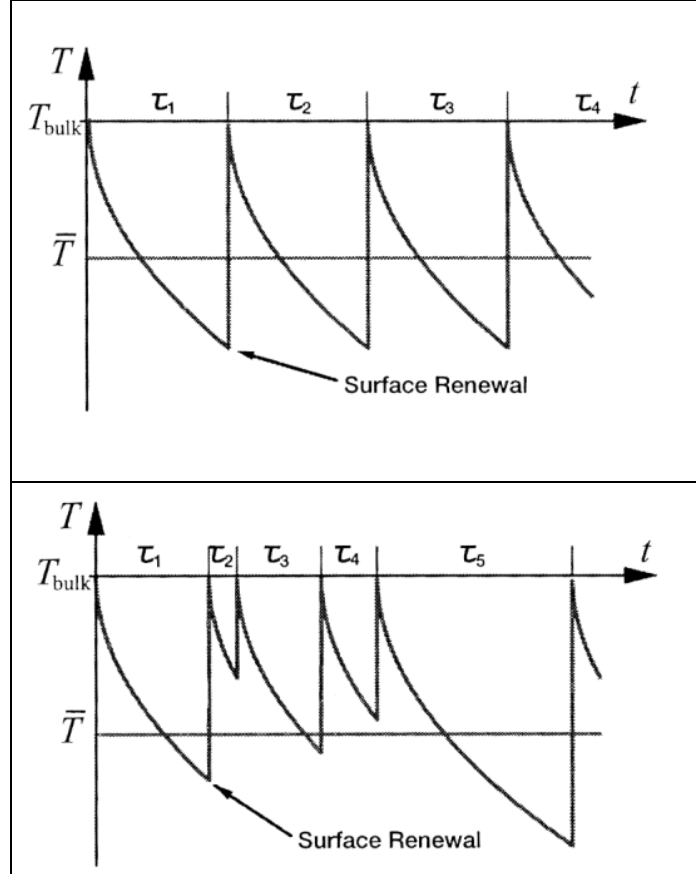

Figure 2: Surface temperature renewal plots

a.) (top) with identical times between renewal events (a single tau)

b.) (bot) with varying taus

(Both images from Garbe, 2001) however, it is assumed that the temperature change due to horizontal advection is relatively small compared to the temporal change, then, the 
temperature of each surface patch is determined solely by the time since the last renewal event. Thus, the spatial distribution of temperatures observed in one image of the sea surface is statistically equivalent to the distribution of the temperature in a time series such as Figure $2 \mathrm{~b}$. That is, a statistical distribution of $\tau$ will explain a spatial distribution of $T$, given the temperature curve as defined in equation [1.1.17].

In order to obtain a spatial distribution, the probability of a water parcel renewed with an interval $\tau$ is defined as $p(\tau)$. Within the renewal interval the temperature varies like [1.1.5]. If the distribution of $\tau$ is assumed to be a logarithmic-normal curve with the probability density of

$$
p(\tau)=\frac{1}{\sigma \tau \sqrt{\pi}} e^{\frac{-\left(\ln \tau-\mu^{2}\right)}{\sigma^{2}}}
$$

the statistical distribution of the surface temperature within one infrared image can be estimated as

$$
p\left(T_{S}\right)=\frac{\Delta T}{\left(\alpha j_{0}\right)^{2}} e\left(\frac{\sigma^{2}}{4}-\mu\right) \operatorname{erfc}\left(\frac{\sigma}{2}-\frac{\mu}{\sigma}+\frac{1}{\sigma} \ln \left(\Delta T / \alpha j_{0}\right)^{2}\right),
$$

(Garbe, 2001) where $\sigma$ is the variance, $\mu$ is the mean, $\Delta T_{\text {is }} T_{B}-T_{S}$, and $\alpha$ is defined as

$$
\alpha=\frac{2}{\rho C_{p} \sqrt{\pi \kappa}}
$$

An example of the theoretical distribution is shown in Figure 3a. Figure 3b shows how a typical observed temperature distribution compares with the theoretical curve. It is apparent from this example that this statistical treatment gives a reasonable fit. 


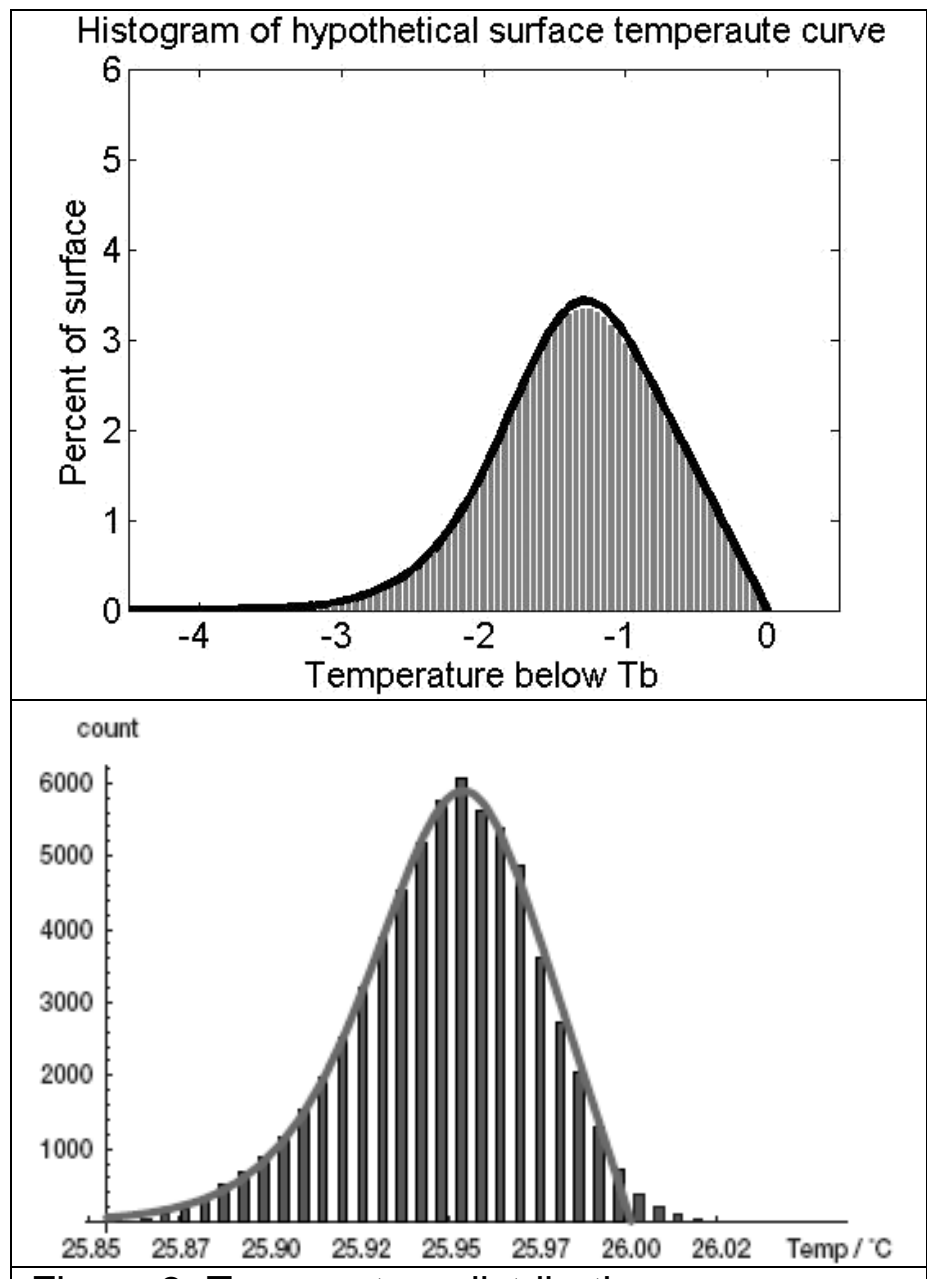

Figure 3: Temperature distributions, hypothetical SRM and observed

a.) (top) A hypothetical temperature distribution based on a tau distribution. The solid black line is equation. [1.2.2]

b.) (bot) An actual application of the SRM to a histogram of observed sea-surface temperatures. The vertical axis, labeled count, refers to the number of pixels at that temperature observed in the image. The grey line is the applied fit of equation. [1.2.2]. (From Garbe, 2001)
Since the temperature depresses with increasing time (and $\tau$ ), this distribution will give a single highest value for the surface temperature distribution. This highest value has been taken to be the "Bulk Temperature" of the well-mixed layer below the cooled surface. One of the weaknesses of this method is that the calculated $T_{B}$ is often lower than the actual measured value. Since the temperature decreases with time, the fact that the bulk temperature is higher than the maximum observed temperature at the surface suggests that the assumption of "instantaneous renewal" may not be correct. This is one of the motivating reasons behind the development of an alternative turbulence model in this study. 


\section{Section 3. Gas Flux Derived from Heat Flux}

Throughout this chapter, most of the discussion has pertained to heat flux, not gas flux. However, the ultimate goal of this research is to apply experimentally obtained knowledge of the heat transfer process to the transfer of gasses. In order to use heat as a proxy tracer, it must be understood how any two tracer fluxes can be compared. Earlier, heat flux was defined in equation [1.1.1]. It is now useful to introduce the related concept of "transfer velocity." Transfer velocity $\left(k_{x}\right)$ is the imaginary velocity of the gas being pushed, as if by a piston, across the boundary layer. Transfer velocity, $k_{x}$, of substance $x$, can be defined as

$$
k_{x}=\beta_{x}^{-1} u_{\star} S c^{-n},
$$

where $\left(\beta_{x}\right)$ is a dimensionless transfer resistance, $u_{*}$ is a friction velocity defined by wind stress $\tau_{w}$ as

$$
\tau_{w}=\rho u_{\star}^{2},
$$

$S_{C}$ is the Schmidt number (or ratio of kinematic to molecular viscosities)

$$
S c=v / D,
$$

and $n$ is $2 / 3$ for smooth seas and $1 / 2$ for rough. For heat, the Prandl number

$$
\operatorname{Pr}=v / \kappa
$$

is used in place of the Schmidt number. If transfer velocity is used, it can be defined in terms of the flux, 


$$
k_{H}=\frac{j_{H}}{\rho C_{p}\left(T_{S}-T_{B}\right)},
$$

where $k_{H}$ is the transfer velocity of heat, $\rho$ is the density of water, $C_{P}$ is the specific heat of water, $T_{S}$ is the surface temperature, and $T_{B}$ is the bulk temperature. For any two tracers, if the transfer velocity of one is known and the values for $D$ (or $\kappa$ ) for both tracers and $n$ are known, the transfer velocity of the other can be obtained by

$$
\frac{k_{a}}{k_{b}}=\left(\frac{S c_{a}}{S c_{b}}\right)^{-n} .
$$

This allows the transfer velocity of a gas to be determined by measuring the transfer velocity of heat and converting the result using equation [1.3.6]. Although, the veracity of this method has been tested by simultaneously measuring the transfer velocities of various tracers, the assumption that heat can be used as a proxy tracer for gases like carbon dioxide is still being debated (e.g., Zappa, et. al. 2004). 


\section{Section 4. Limitations of the Surface Renewal Model}

Even though the Surface Renewal Model is able to describe the surface conditions statistically, it is still limited by the lack of an explanation for the renewal event itself. Images of the surface field show that the patches tend to remain coherent for longer than the average renewal time-scale. Although the Surface Renewal Model assumes that renewal events happen instantaneously, this cannot be physically correct. Even breaking waves take some measurable amount of time to stir up the warmer waters through induced turbulent action. This time will allow cooling or mixing to occur, reducing the observed surface temperature.

Another limit of the Surface Renewal Model is that it assumes the bulk temperature to be identical to the highest measured temperature on the surface. If waters advected up do not arrive instantaneously (i.e. renewal is not instantaneous), some cooling will have taken place by the time bulk waters reach the surface. The determination of this cooling, however, is precluded by the assumption that the renewal is instantaneous.

There is also a question as to whether the relation of transfer velocities is as straight-forward as in equation [1.3.6]. The question arises from the fact that the fluxes are calculated differently; the heat is considered to have a constant surface flux, whereas the gas flux is controlled by the constant surface concentration. Some researchers (e.g., Zappa, et. al. 2004) have 
questioned whether this relation is a valid one based on the relative differences between the values.

Lastly, in the infra-red images, the turbulence is often visualized as streaks of cool water and patches of warm. It appears as though the images are showing the surface signature of the Langumir turbulence. If the turbulence is being detected, it could be possible to solve for this motion explicitly, without treating the event as an unknown instantaneous event. However, a new mechanistic and mechanical model would be needed to explain this motion. 


\section{Chapter 2. The Eddy Renewal Model}

\section{Section 1. Eddy Renewal Model - Upwelling Solution}

The Surface Renewal Model assumes that temperature change due to advection is zero and that the renewal is an instantaneous event. In the event of wave breaking processes, these may be reasonable assumptions since vertical motions due to breaking events occur quickly compared to the typical renewal time scale. However, the infrared images tend to show coherent temperature patterns, suggesting that the renewal processes are also persistent. Therefore, it may be possible to have a model where bulk water is constantly being advected up, spreading out along the surface (within the diffusive sublayer), and then downwelling back into the well mixed layer.

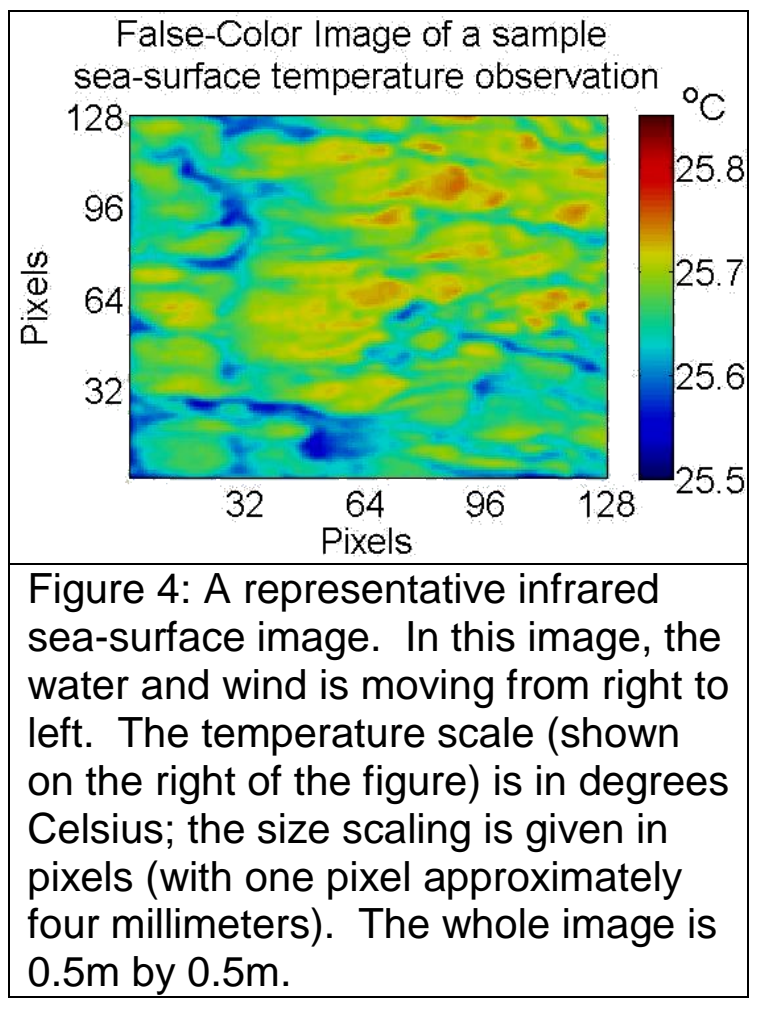

This would allow the advected water to be cooled by molecular diffusion of heat while being advected to the surface.

In viewing the images collected (see Figure 4 for a typical sea-surface temperature image), it appears that distinct turbulence patterns exist at the surface, namely, Langumir turbulence. This is shown in elongated patches 
of warm water alternating with cold water filaments, with axes aligned with the predominant wind direction. From this, it can be assumed (for the sake of easing calculations) that, if the long axis is selected as $y, \partial / \partial y$ is negligible and can be set to zero. Scaling arguments can be used to show that $\partial / \partial x$ is also negligible in the diffusion term. Lastly, since the surface patches tend to exist for a relatively long time, $\partial / \partial t$ is also considered zero. These assumptions reduce equation [1.1.6] to

$$
u \frac{\partial T}{\partial x}+w \frac{\partial T}{\partial z}=\kappa \frac{\partial^{2} T}{\partial z^{2}}
$$

or, in the case of a gas concentration,

$$
u \frac{\partial C}{\partial x}+w \frac{\partial C}{\partial z}=D \frac{\partial^{2} C}{\partial z^{2}} .
$$

In order to solve equations [2.1.1] and [2.1.2], the surrounding modeled fluid motions must be considered, as well as the boundary (surface and depth) conditions. If the surface turbulence is visualized as Langumir-type eddies (Figure 5), this gives the general physical

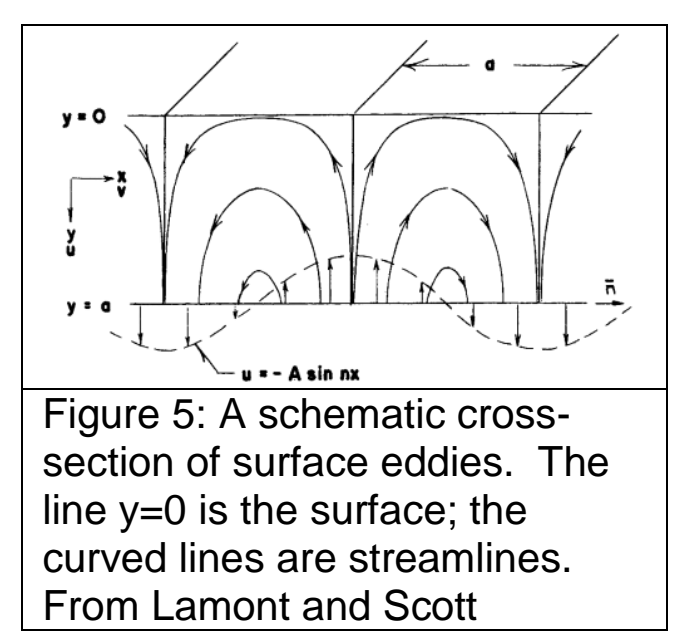
description of long "rollers" spinning next to each other, but in alternating directions. A cross-section of this schematic would resemble Figure 5. As first described by Lamont and Scott (in 1970), there are alternating regions of upwelling and downwelling, with an overall structure like that of a series rollers, with alternating eddies spinning in opposite directions. (When 
temperature is added to the model, this equates to regions of warm water upwelling, cooled water downwelling and regions of fairly constant temperature between.) Using Taylor expansions, first order approximations for $w$ and $u$ are obtained near the surface as

$$
\begin{aligned}
& w=-\left.z \frac{\partial u}{\partial x}\right|_{x=0}+O\left(z^{2}\right)+\ldots, \\
& u=\left.u\right|_{x=0}+O(z)+\ldots
\end{aligned}
$$

and

$$
u_{s} \equiv u(z=0) \text {. }
$$

Combining equations [2.1.2], [2.1.3], [2.1.4], and [2.1.5], we obtain

$$
u_{s} \frac{\partial T}{\partial x}-z \frac{\partial u_{s}}{\partial x} \frac{\partial T}{\partial z}=\kappa \frac{\partial^{2} T}{\partial z^{2}} .
$$

In the center of the upwelling region, where $u_{s}=0$, a particular solution is available when the horizontal velocities become zero. Equation [2.1.1] becomes

$$
w \frac{\partial T}{\partial z}=\kappa \frac{\partial^{2} T}{\partial z^{2}} .
$$

Using the approximation from equations [2.1.3] and [2.1.5],

$$
w=-z \frac{\partial u_{s}}{\partial x} .
$$

If the horizontal surface velocity is assumed to be linear near the upwelling points, the approximation of

$$
u_{s}=S x
$$


is valid. This, combined with equation [2.1.8] gives

$$
w=-S z
$$

Equation [2.1.7] can thus be written as

$$
-S z \frac{\partial T}{\partial z}=\kappa \frac{\partial^{2} T}{\partial z^{2}} .
$$

If the heat flux is defined as

$$
j_{H}=\kappa \frac{\partial T}{\partial Z},
$$

the differential equation for the flux is

$$
-S z j_{H}=\kappa \frac{\partial j_{H}}{\partial z} .
$$

Through rearranging and integration, the solution for flux is obtained as

$$
j_{H}=j_{H(z=0)} \exp \left(-Z^{2} \frac{S}{2 \kappa}\right),
$$

where $j_{H(z=0)}$ is the surface (heat) flux at the upwelling point. This equation can be integrated once to yield a temperature profile of

$$
T=\left(T_{S}-T_{B}\right) \operatorname{erfc}\left(\frac{-\sqrt{S}}{\sqrt{2 \kappa}} Z\right)+T_{B} .
$$

Up to this point, the solutions for heat and gas transfer would be identical, with the only difference being the appropriate constants and symbols. For instance, equation [2.1.14] would be written for gas as

$$
j_{g}=j_{g, z=0} \exp \left(-z^{2} \frac{S}{2 D}\right),
$$

and equation [2.1.13] would be written as 


$$
-S z j_{g}=D \frac{\partial j_{g}}{\partial z}
$$

where $j_{g}$ is the gas flux. The solution for the concentration is

$$
C=\left(C_{S}-C_{B}\right) \operatorname{erfc}\left(\frac{-\sqrt{S}}{\sqrt{2 D}} z\right)+C_{B} .
$$

These specific solutions have a similar form to the solution obtained for the Surface Renewal Model. However, this is only applicable to the upwelling region. The solutions away from the upwelling point differ depending on the surface boundary conditions. 


\section{Section 2. Eddy Renewal Model - Eddy Interior Solution}

In order to solve equation [2.1.1] for the interior of the eddy, it is mathematically easier to transform the coordinates. The horizontal and vertical axis will be transformed such that

$$
\begin{aligned}
& \Xi=\int_{0}^{x} u_{s}\left(x^{\prime}\right) d x^{\prime} \\
& Z=z \cdot u_{s}(x)
\end{aligned}
$$

which transforms the coordinates $(x, z)$ into $(\Xi, Z)$.

Transforming equation [2.1.6] requires rewriting the partial derivatives

as

$$
\begin{aligned}
& \frac{\partial}{\partial x}=\frac{\partial}{\partial \Xi} \frac{\partial \Xi}{\partial x}+\frac{\partial}{\partial Z} \frac{\partial Z}{\partial x}=\frac{\partial}{\partial \Xi} u_{s}+\frac{\partial}{\partial Z}\left(z \frac{\partial u_{s}}{\partial x}\right) \\
& \frac{\partial}{\partial z}=\frac{\partial}{\partial \Xi} \frac{\partial \Xi}{\partial Z}+\frac{\partial}{\partial Z} \frac{\partial Z}{\partial z}=\frac{\partial f^{0}}{\partial \Xi}+\frac{\partial}{\partial Z} u_{s} .
\end{aligned}
$$

Combining these with equation [2.1.6], we obtain

$$
u_{s} u_{s} \frac{\partial T}{\partial \Xi}+u_{s} \frac{\partial T}{\partial Z} z \frac{\partial u_{s}^{0}}{\partial x}-z \frac{\partial u_{s}}{\partial x} u_{s} \frac{\partial T^{0}}{\partial Z}=\kappa u_{s}^{2} \frac{\partial^{2} T}{\partial Z^{2}}
$$

which reduces to

$$
\frac{\partial T}{\partial \Xi}=\kappa \frac{\partial^{2} T}{\partial Z^{2}}
$$

and 


$$
\frac{\partial C}{\partial \Xi}=D \frac{\partial^{2} C}{\partial Z^{2}}
$$

as the equation for the gas. These equations, of course, look surprisingly similar to equation [1.1.7]. However, to be able to compare equations [2.2.6] and [2.2.7] to the solutions obtained for the upwelling regions, equations [2.1.18] and [2.1.15], surface boundary conditions must be taken into account. The easier solution of the constant surface concentration will be shown first.

For the case where there is a constant surface parameter (i.e. gas), the general solution of equation [2.2.7] can be obtained through the use of surface and bulk concentration definitions as before, yielding

$$
C=\left(C_{S}-C_{B}\right) \operatorname{erfc}\left[\frac{-Z}{2 \sqrt{D \Xi}}\right]+C_{B} .
$$

In order to compare this solution to that for the upwelling region, it will need to be reverted back to the $(x, z)$. By simple substitution of equations [2.2.1] and [2.2.2] into [2.2.8], the form for the gas concentration solution in $(x, z)$ is

$$
C=\left(C_{S}-C_{B}\right) \operatorname{erfc}[\zeta]+C_{B}
$$

where

$$
\zeta=\frac{-z u_{S}}{2 \sqrt{D \int_{0}^{x} u_{S}\left(x^{\prime}\right) d x^{\prime}}}
$$

Using the approximations [2.1.9] near the upwelling point,

$$
\int_{0}^{x} u_{S} d x=\frac{1}{2} S x^{2}
$$

which simplifies $\zeta$ as 


$$
\zeta=\frac{-z S x}{2 \sqrt{D \frac{1}{2} S x^{2}}}=\frac{-z \sqrt{S}}{\sqrt{2 D}},
$$

giving the general solution the identical form to the upwelling solution found in equation [2.1.18]. Thus [2.2.9] is a valid solution for the entire space.

The solution for the constant surface flux boundary condition requires a slightly different approach. Using $\mathrm{j}_{\mathrm{H}, \mathrm{O}}$ as the heat flux at the surface for all $\mathrm{X}$,

$$
j_{H, 0}=\frac{\partial T}{\partial z} .
$$

Or, in the transformed coordinates,

$$
j_{H, 0}=\frac{\partial T}{\partial Z} u_{S} .
$$

If us is assumed to be a sine function (which does not invalidate the approximation of equation [2.1.9] that us is linear close to where us $=0$ ), as in

$$
u_{s}=\sin (\tilde{k} x)
$$

where $\tilde{k}$ is the length scale of the eddy and $\mathrm{x}=0$ is the center of the upwelling. Using equation [2.2.1], $\Xi$ can be defined as

$$
\Xi=\frac{-1}{\tilde{k}} \cos (\tilde{k} x)+\frac{1}{\tilde{k}}=\frac{1}{\tilde{k}}(1-\cos (\tilde{k} x)) .
$$

This allows us to be redefined in terms of $\tau$ as

$$
u_{s}=\sin \left(\cos ^{-1}(1-\Xi \tilde{k})\right)
$$

Thus, the vertical temperature gradient in the transformed coordinates at the surface of the air-water interface can be expressed as 


$$
\frac{\partial T}{\partial \mathrm{Z}}=\frac{j_{H, 0}}{\sin \left(\cos ^{-1}(1-\Xi \tilde{k})\right)} .
$$

This gives the boundary condition that can be used to solve [2.2.6] numerically, if not analytically. 
Section 3. Normalization and Solution of the Temperature and $\underline{\text { Concentration Fields }}$

In further examining the model presented in previous sections, it is apparent that there are three parameters that define the characteristics of a particular eddy, namely, the size of the eddy, the intensity of the motions found within the eddy, and the bulk temperature. However, mathematically, only the intensity and eddy size determine the shape of the temperature distribution, while the bulk temperature sets the specific value of the temperature curve. The intensity and eddy size can be treated as one parameter measuring the surface divergence, as shown below.

Starting from the equation of temperature diffusion, and using the same assumptions as before,

$$
\begin{aligned}
& u \frac{\partial T}{\partial x}+w \frac{\partial T}{\partial z}=\kappa\left(\frac{\partial^{2} J^{0}}{\partial x^{2}}+\frac{\partial^{2} T}{\partial z^{2}}\right) \\
& u=u_{0} \sin (\tilde{k} x)+\text { H.O.T. } \\
& w=-\int \frac{\partial u}{\partial x} d z=-\int u_{0} \tilde{k} \cos (\tilde{k} x) d z=-u_{0} \tilde{k} \cos (\tilde{k} x) \cdot z \text {, and } \\
& -\kappa \frac{\partial T}{\partial z}=\left.j_{0}\right|_{z=0}
\end{aligned}
$$

These equations can be normalized by introducing the following normalized variables:

$$
\begin{aligned}
& \tilde{x}=\tilde{k} x, \\
& \tilde{z}=\frac{z}{\delta}, \text { and }
\end{aligned}
$$




$$
\tilde{T}=\frac{T k}{\delta j_{0}}
$$

where $\delta^{-1}$ is the boundary layer scale, $\tilde{x}, \tilde{z}$, and $\tilde{T}$ are scaled horizontal and vertical dimensions and temperature, respectively, and $\tilde{k}$ is the wavenumber of the eddy. Equation [2.3.1] can be rewritten as

$$
\frac{\partial \tilde{T}}{\partial \tilde{x}}\left(\tilde{k} u_{0}\right) \sin (\tilde{x})-\frac{\partial \tilde{T}}{\partial \tilde{z}} u_{0}(\tilde{k} \tilde{z}) \cos (\tilde{x})=\kappa \frac{\partial^{2} \tilde{T}}{\partial \tilde{z}^{2}} \frac{1}{\delta^{2}} .
$$

If the boundary layer scale in equation [2.3.6] is defined in terms of the intensity of the eddy motions,

$$
\tilde{k} u_{0}=\kappa \delta^{-2},
$$

equation [2.3.8] can be simplified to

$$
\sin (\tilde{x}) \frac{\partial \tilde{T}}{\partial \tilde{x}}-\cos (\tilde{x}) \tilde{z} \frac{\partial \tilde{T}}{\partial \tilde{z}}=\frac{\partial^{2} \tilde{T}}{\partial \tilde{z}^{2}}
$$

giving one equation for the temperature distribution that is independent of the size of the eddy. The advantage of this is three-fold. First, equation [2.3.10] gives the means by which the temperature field may be obtained (computationally, if not analytically). Second, this computed temperature field is then scaled by the observed to obtain a modeled field that matches the observed eddy temperatures. And third, the parameter $\left(\tilde{k} u_{0}\right)^{-1}$ (which has the units of time) can be thought of as the residence time that a parcel spends on the surface (or the inverse of surface divergence). This parameter can be directly compared to the parameter of $\tau$ used in the Surface Renewal Model. This will be further discussed in following sections. 
By using the above equations and coding in mathematical analysis software (such as MatLab), generalized solutions for the temperature field and gas concentration field were obtained. These solutions can be compared to the known solutions for the Surface Renewal Model. The basic structures of these fields deserve some discussion (and are presented in Figure 6).

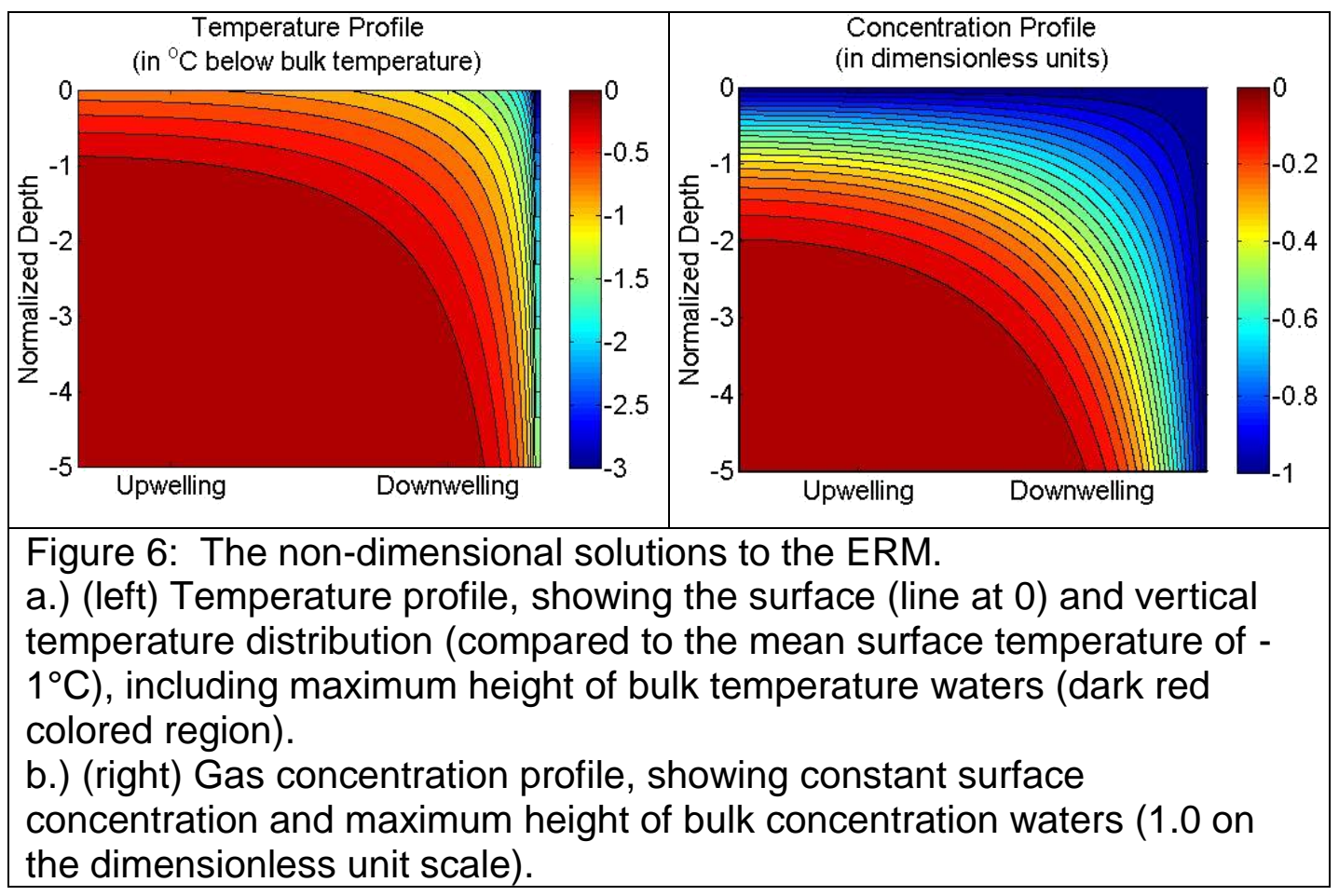

The temperature solution obtained through computation (Figure 6a) shows an eddy, in which warm waters are advected up to the surface (on the left), along the surface (from left to right) and then down (on the right). Rather than the motion of the water being described by an instantaneous event, the motion is described as a continuous flow from upwelling to downwelling. Once the waters have traveled sufficiently far from the surface, it is assumed that the turbulent motions of the mixed layer will blend the downwelled waters with the existing mixed layer. Since the difference in scale between the 
mixed layer and the surface-cooled layer is enormous, it is appropriate to assume that the mixed layer is infinite, for the purposes of these calculations.

There is an inherent anomaly in the temperature model presented above. At the downwelling point, solution becomes unbounded; the temperature decreases indefinitely as the water approaches this point. This can only be observed in analyzing the equations (such as equation [2.3.10]); Figure $6 a$ does not show this anomaly due to the limitations of the computational analysis and pixel / matrix size. In reality, very close to the downwelling point the previously neglected terms (e.g., the horizontal diffusion and gradient terms) become important. A more full solution of the temperature equation must be solved to accurately model the downwelling region. This was not pursued in this study since this region is small enough that, when the modeled surface temperature curve is applied to the images (as described later), the effects are minimal.

It should also be noted that there is an underlying assumption that these eddies are paired with a mirrored eddy. That is, for every eddy rotating with a positive spin, there is one next to it rotating with a negative. This gives a symmetrically decreasing temperature profile (moving away from the center). This form is qualitatively consistent with the temperature signal of warm patches surrounded by cool streaks, as obtained from infrared images of the air-water interface.

Thus, this model presents an image of surface temperature renewal where the surface motions are described as eddy-like, and these motions 
control the length of time any one parcel of water remains at the surface. With the assumption of a constant surface heat flux, the length of time at the surface controls the cooling of the waters, and thus the temperature. Given the above normalizations, the basic temperature pattern can be applied to the observed data by scaling the modeled pattern according to the unique values of the bulk temperature and the surface divergence.

The gas solution of the Eddy Renewal model (Figure 6b) has some similarities to the gas solution found using the Surface Renewal Model (Figure 1a). There is a constant surface concentration, and there is a deepening of the decreased concentration near the surface further away from the upwelling / renewal point. However, at the renewal point in the SRM, the vertical concentration gradient becomes unbounded; even though the surface concentration remains constant, according to the assumptions previously discussed, the limit as $z \rightarrow 0$ and $t \rightarrow 0$ of equation [1.1.11] is that the surface temperature is equal to the bulk temperature. Thus, in the SRM, the bulk concentration waters practically emerge at the surface. In the Eddy Renewal Model gas solution, the bulk concentration is never observed near the surface. However, at the downwelling point, the surface concentration is carried down to the bulk layer. There is also a general difference in the shape of the two profiles, similar to what is discussed for the temperature profiles. The effects of the different gas concentration profiles on the gas transfer problem have not yet been explored. 


\section{Chapter 3. Comparison of Models}

\section{Section 1. Comparison of Model Characteristics}

One immediately obvious difference between the Eddy Renewal Model and the Surface Renewal Model is that the bulk temperature is never observed on the surface of the eddy in the former. This difference between the models can be easily explained. In the surface renewal model, the process that transfers water to the surface is assumed to be an instantaneous process. That is, no time is given for the bulk waters advected to the surface to mix and/or cool. The continuous eddy model allows for bulk water to be cooled as it is advected closer to the surface. The strength of the cooling is based on (and inverse to) the strength of the vertical motions of the eddy.

A more quantitative direct comparison of the two models will be achieved in two steps. First, a single renewal event in the Surface Renewal Model is compared with a single eddy in the Eddy Renewal Model (using identical parameter values for eddy size, surface flux, and mean and bulk temperatures, and comparing the resulting values for renewal time scales and surface divergence). And second, the application of a distribution of renewal time scales in the surface renewal model is compared with the application of the identical distribution of the surface divergence of eddies in the eddy renewal model. 


\section{Section 2 Single Renewal Event / Eddy Comparison}

In order to compare the two models, two sample regions are created and analyzed. The regions are of equal size, and have equal mean surface

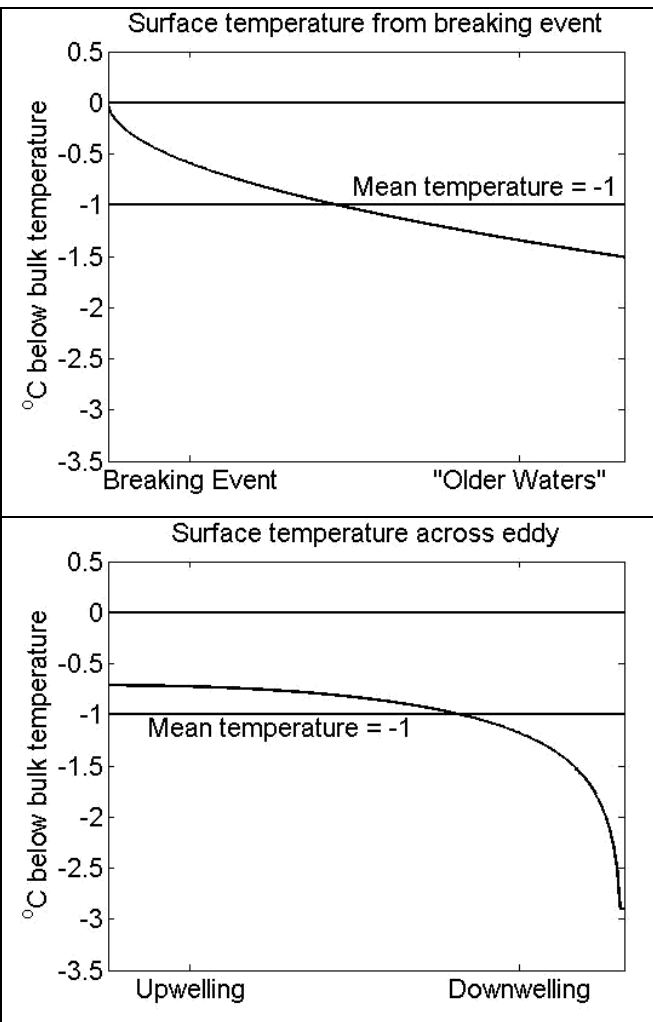
temperatures curves for similar regions of SRM and ERM, with matching mean temperatures. a.) (top) Surface temperature decay as a function of distance from breaking event (Temporal) b.) (bot) Surface temperature decay as a function of distance from upwelling region (Spatial)
Figure 7: Comparison of surface

temperature (-1) as well as equal bulk temperature (0). In one region (using the Surface Renewal Model), it is assumed that a breaking front moves through the region at a constant speed, traveling from right to left in the model eddy. At the time of the "snapshot," the wave has just left the system. Therefore, the horizontal axis, which is the distance from the breaking front, is proportional to the time since the last renewal event. The sample eddy for the Eddy Renewal Model is taken to be the region from one upwelling zone (on the left) to one downwelling zone (on the right). The sample region for the Eddy Renewal Model is only one eddy in a paired system of eddies (i.e. half of a warm patch). These regions are selected in order to show higher temperatures on the left and lower 
temperatures on the right of both models. The temperature distributions of the two eddies show the difference in the cooling between the models (in Figures $7 a$ and $b)$.

In the temporal model, as the breaking event moves through the area, it stirs up bulk temperature water, which then cools off (as a function of the time). In the spatial model, waters begin their cooling while they are advecting towards the surface; they continue their cooling as they move along the surface, and eventually sink. However, in the spatial model, waters from below the center of the eddy allow for decreased surface temperature, due to an increased heat flux from waters at depth. This spatial temperature distribution seems to be more in line with the observed patches of warm, homogenous water surrounded by outlines of intensely colder waters.

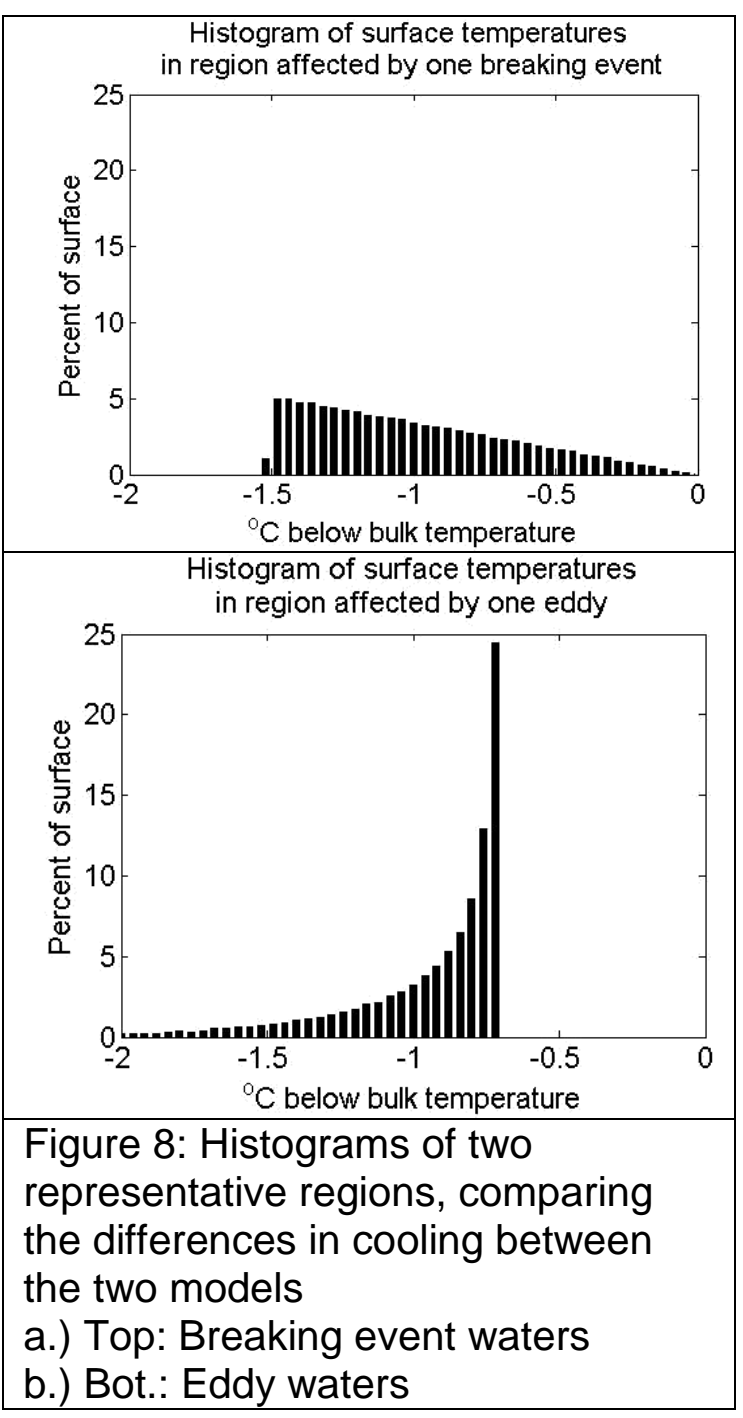

Another way to interpret the difference between the models is to compare histograms of the surface temperatures (Figure 8). This will be most useful because the previous research based on the Surface Renewal Model 
has used histograms of ocean surface images to validate the approximation. Once again, the difference between the two models is striking. In the Surface Renewal Model, it is clear that the waters reach colder temperatures more quickly than they do in the Eddy Renewal Model. However, the maximum value of the bulk temperature is never observed at the surface in the Eddy Renewal Model.

In order to compare the vertical temperature distributions, one would need to match not only the size of the region, the bulk temperature, and the mean surface temperature, but also the surface heat flux. For instance, if the size is taken to be $5 \mathrm{~cm} \times 5 \mathrm{~cm} \times 1 \mathrm{~mm}$, the surface flux is set as $100 \mathrm{~W} / \mathrm{m}^{2}$, and the mean surface temperature is arbitrarily defined as $0.1^{\circ} \mathrm{C}$ below the bulk temperature, one would obtain (through proper scaling) the results in Figure 9. From these images, it is easy to see how the two models differ. In the Surface Renewal Model (Figure 9a), the bulk temperature waters are actually brought to the surface (but only at the renewal event), while in the Eddy Renewal Model (Figure 9b), the bulk temperature waters are cooled before they advect to the surface. In the ERM, the temperature depression extends below the surface at all locations in the sample volume; this is not true for the SRM. The motion of the turbulence in the water can be inferred in the ERM as upwelling where there is a decreased thickness of the cooled waters and downwelling where the cooled waters extend far below the surface. 

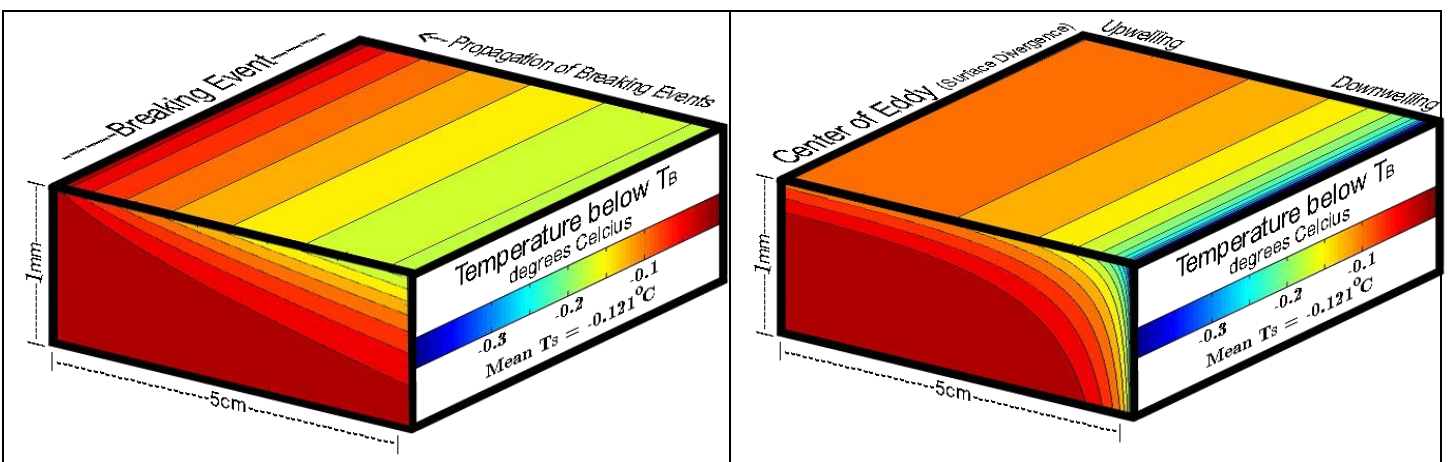

Figure 9: Representational volumes of water acted upon by the two different models. Both volumes have matching size $(1 \mathrm{~mm}$ in the vertical and $5 \mathrm{~cm}$ in the horizontal), jo $\left(100 \mathrm{~W} / \mathrm{m}^{\mathrm{s}}\right), \mathrm{Cp}\left(3850 \mathrm{~J} \mathrm{~kg}^{-1}{ }^{\circ} \mathrm{C}^{-1}\right), \rho\left(1025 \mathrm{~kg} \mathrm{~m}^{-3}\right)$, and mean surface temperature $\left(0.121^{\circ} \mathrm{C}\right.$ below bulk temperature).

a.) (left) Water acted upon by the SRM; $\tau$ value is 5.50 seconds.

b.) (right) Water acted upon by the ERM; $1 / \tilde{k} u_{0}$ value is 1.00 seconds.

Lastly, it is of interest to note the values obtained for the two comparative parameters, $\tau$ and $1 / \tilde{k} u_{0}$. Given the parameters for these specific volumes, one finds that the value for $\tau$ is 5.50 seconds, while that for $1 / \tilde{k} u_{0}$ is 1.00 seconds. Therefore a spatial eddy with a time scale of $1 / \tilde{k} u_{0}$ is comparable to a surface renewal event with a time scale $\tau$ that is 5.50 times the value of $1 / \tilde{k} u_{0}$ since they yield the same heat flux for the same temperature difference between the surface and bulk waters. 


\section{Section 3. Comparison of Model Parameters}

In order to compare the temperature distribution of a large number of eddies, to a large number of surface renewal events, a single scaling parameter for each model must be used.

In the SRM, the standard method of relating the spatial temperature distribution to the surface temperature changes over time [1.1.17] is to assume that each parcel of water observed at the water (in terms of measurements, each pixel on an image) remains at the surface for a total period of $\tau$ and that there is an equally likely chance of finding a parcel that has remained at the surface from 0 to $\tau$ seconds. It is further assumed that the values for $\tau$ have a logarithmic-normal distribution as defined by the probability density curve in equation [1.2.1] and the parameters in [1.2.1] are functions of the environment (perhaps wind-speed or wave-slope spectra).

In the Eddy Renewal Model, the equations were normalized based on two parameters: $\tilde{k}$ and $u_{0}$, the size and strength, respectively, of the eddy. From equation [2.3.5], $\tilde{k}$ is the length-scaling parameter. That is, the inverse of $\tilde{k}\left(\tilde{k}^{-1}\right)$ can be thought of as the length of the eddy. The strength of the eddy, $u_{0}$ (from equation [2.3.2]), is the maximum velocity observed at the surface. The parameter $\tilde{k} u_{0}$ is the surface divergence of the eddy, and it is this parameter that can be used to examine the temperature distributions over a range of eddies. Analyzing the dimensions of these two parameters, one finds that $\tilde{k} u_{0}$ has dimensions of (time $)^{-1}$. 
This parameter is a measure of how much the surface diverges from the upwelling point. The strength of this divergence is what controls the observed surface temperature fields. That is, for one given value of $\tilde{k} u_{0}$, there will be one temperature distribution (spread over the size of the eddy). The inverse of $\tilde{k} u_{0}$ can also be thought of as the time it takes for a parcel of water to travel from the upwelling region to the downwelling region, or the time scale of the eddy. Thus, just as $\tau$ is the parameterized residence time of a parcel of water in the SRM, $1 / \tilde{k} u_{0}$ can be thought of as the residence time of a parcel of water in the ERM. In fact, as was demonstrated in the previous section, a single spatial eddy with a surface divergence of $1 / \tilde{k} u_{0}$ is comparable to a single renewal event with renewal time scale $\tau$ that is 5.50 times the value of $1 / \tilde{k} u_{0}$.

Therefore, the overall surface temperature distributions can be compared between the models if comparable distributions of $\tau$ for the SRM and $1 / \tilde{k} u_{0}$ for the ERM are introduced and applied to the single temperature curve. 


\section{Section 4. Comparison of Identical Parameter Distributions}

Using the previous results for the spatial temperature curve (Eddy Renewal Model) and the temporal temperature curve (Surface Renewal Model), it is possible to compare a distribution of eddies to a distribution of renewal times. This allows the comparison of the two models over a region encompassing more than just one sample eddy and renewal event.

When identical distributions of the parameters are applied to the meannormalized surface temperature curves, the resulting surface temperature distributions are very different. As discussed in section two of chapter one, the temperature distribution obtained for the SRM with a log-normal distribution of $\tau$ is shaped like the plot in Figure 10a and described by equation [1.2.2]. However, when the same distribution is applied to a meannormalized ERM surface temperature curve, the resulting temperature distribution is much different (Figure 10b). For quick reference, the difference between these plots can be seen in Figure 10c.

This difference in temperature distributions comes about from the differences in the surface temperature curves. The SRM curve starts from the bulk temperature and decreases with time up to the renewal time of $\tau$ (as seen in the histogram in Figure 8a). This gives a lowest temperature as

defined by each $\tau$. The ERM curve highly favors the mean value, has a defined maximum temperature value lower than the bulk temperature, and 
has no clearly defined lowest temperature value (as seen in the histogram in Figure 8b).

Thus, even though the identical distribution of time scale parameters was applied to the mean-normalized surface temperature curves, the resulting temperature distributions are rather different.
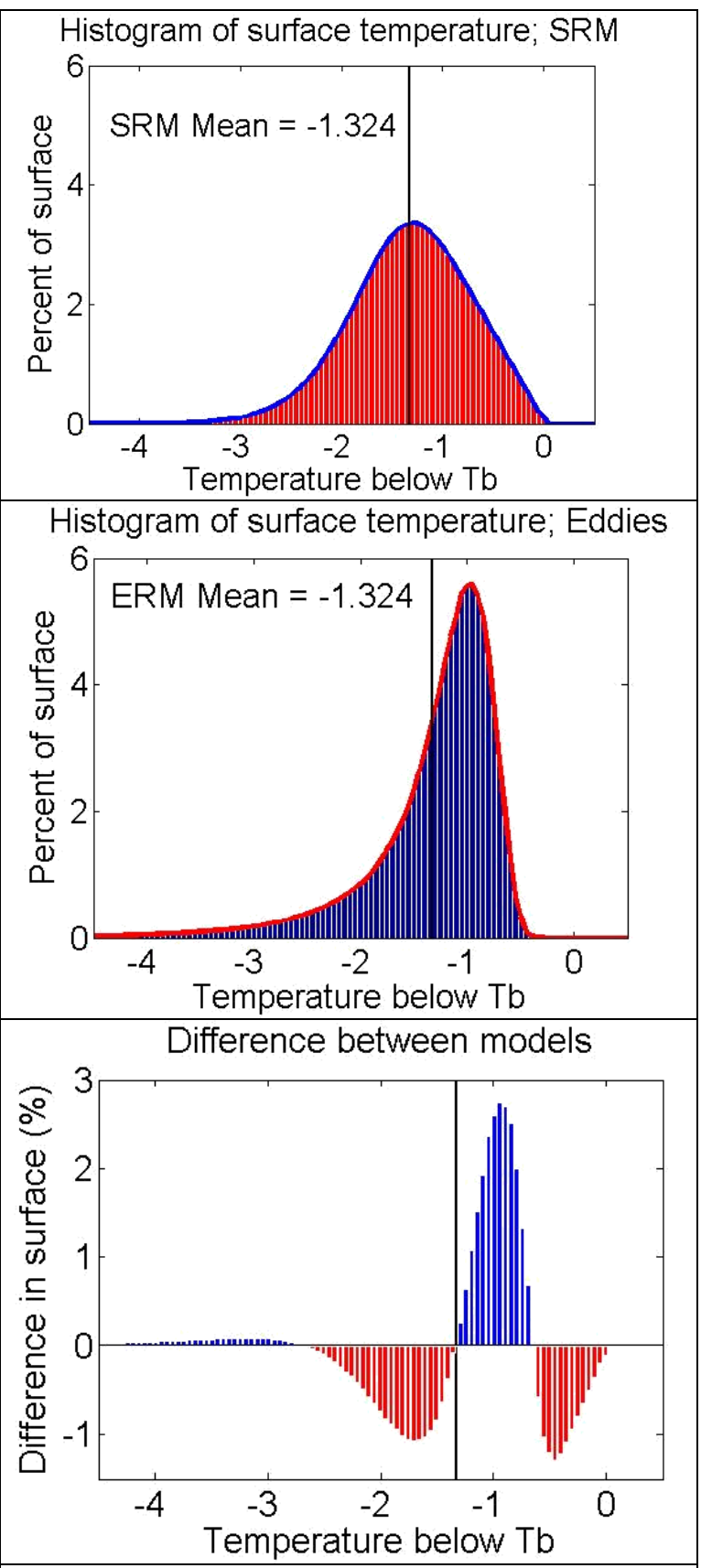

Figure 10: Comparison of Temperature Distributions

a.) (top) Distribution of SRM parcels

b.) (mid) Distribution of eddies

c.) (bot) Difference between the models. Specifically, ERM histogram minus SRM. 


\section{Chapter 4. Applying the ERM to Infrared Sea-Surface Images}

Section 1. Methodology of Image Analysis

In order to determine the validity of the Eddy Renewal Model surface
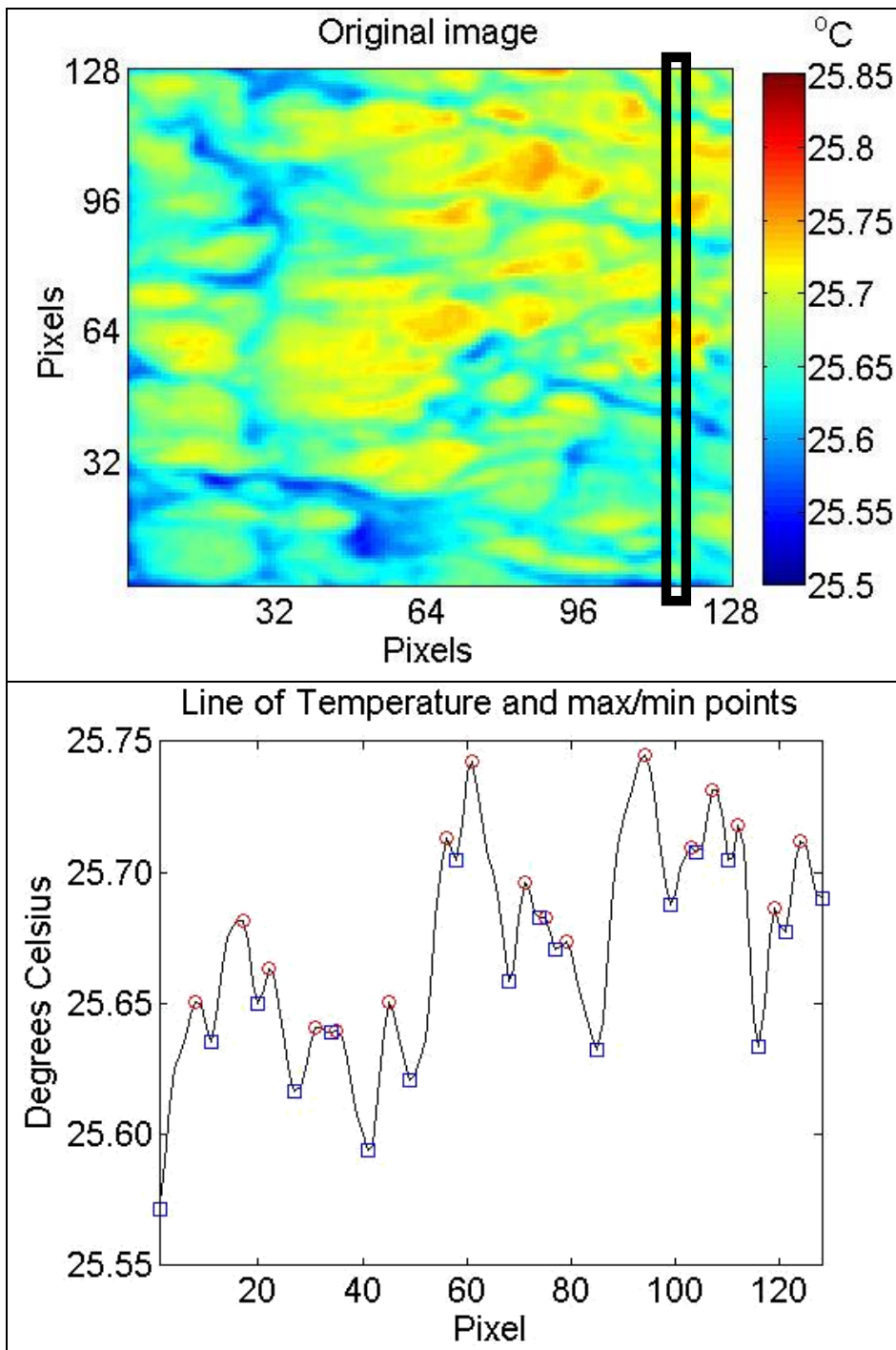

Figure 11: Finding the local maxima \& minima a.) A representative infrared image of the sea surface taken during the GASEX2001 cruise, and a line of data to be analyzed.

b.) The temperature along the line in $11 a$, with the local maxima and minima identified. temperature curve (as

in Figure $7 b)$, the curve

will be applied to actual

infrared images of the

water surface. As

discussed earlier, one of the basic physical constructs of the Eddy Renewal Model is twinned eddies with alternating spin aligned with the predominant wind direction and uniform along the axis of rotation. Even though the real eddies are, of course, nonuniform in the wind direction, the assump- 
tion may still be justified since the observed temperature patterns tend to show more variation in the cross wind direction than in the along wind direction (Figure 11a). Therefore the ERM is applied to the surface in a direction that is nearly perpendicular to the predominant wind direction, as shown in Figure 11b. The application of the model along one column of data allows the fit of the theoretical temperature curve to be individualized for each eddy, as explained below.

Several methods were tested to optimize this matching. The bulk temperature, as the most basic parameter in the model, must first be defined. Initially, the bulk temperature was defined simply as the highest recorded surface temperature, much as in the modeled SRM field. Using a single value for the bulk temperature for the entire image led to poor model / data fits. It was found that the fit of the model to the data could be much improved by allowing the bulk temperature to vary throughout the image. These variations were created by matching the mean temperature of the model to the mean temperature of each eddy, then allowing the bulk temperature to be found by a least-squares best fit analysis. Two different methods of bulk temperature variation were tested: dividing each image in to subsections and defining the bulk temperature within each subsection (i.e. assuming constant bulk temperature within each subsection) and allowing the bulk temperature to be defined for each eddy analyzed (in each line of analysis).

From a physical perspective, the former would make more sense; that is, any variation of the temperature in the well mixed layer should occur over 
a region much larger than an individual eddy scale. However, when the bulk temperatures were allowed to vary eddy by eddy and line by line, it was found that the mean of such bulk temperature estimates over a subsection of the image was very close to the single bulk temperature estimate for the same

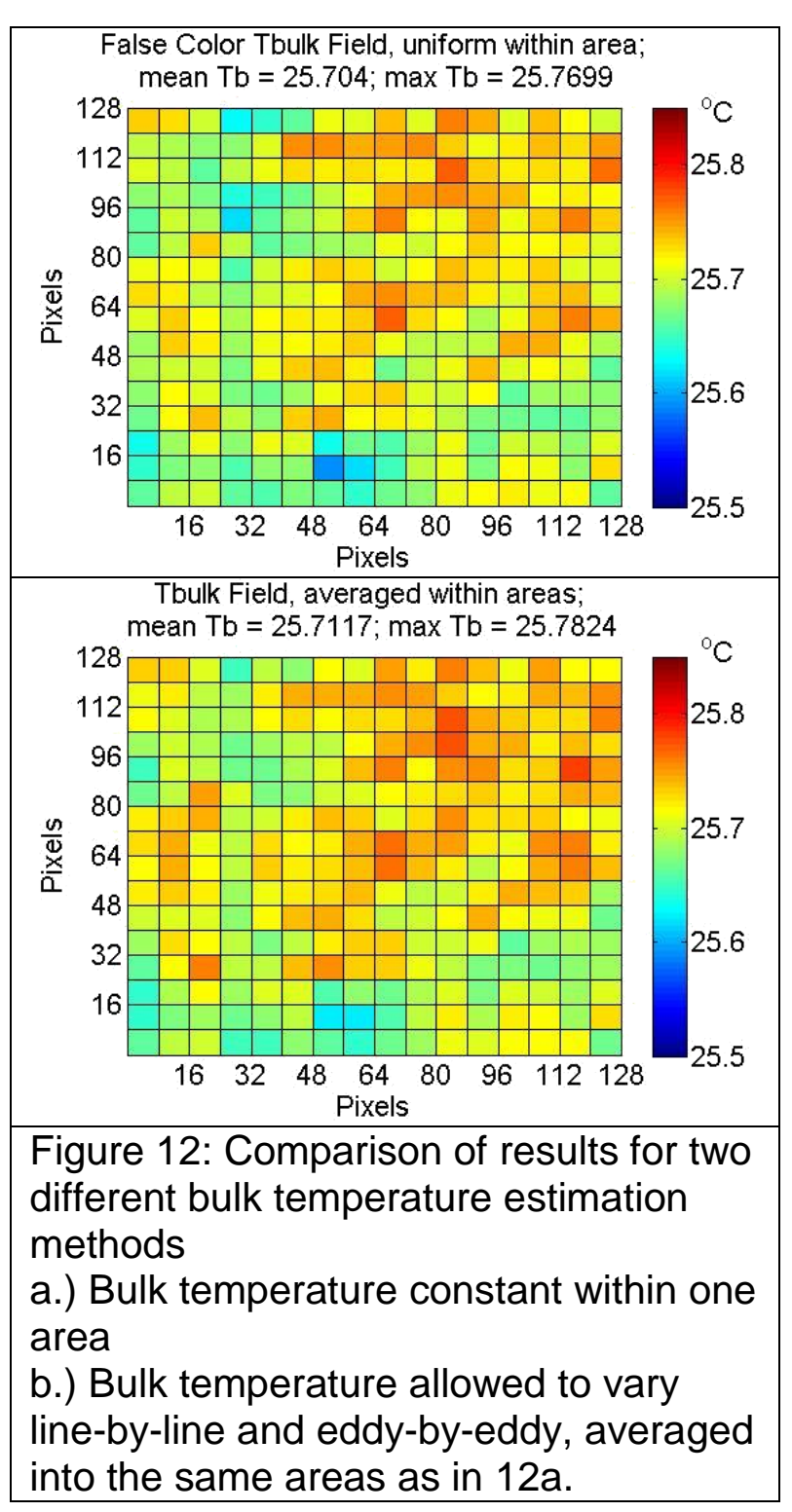

region assuming that the bulk temperature was uniform. (See Figure 12.) Thus, the bulk temperature was allowed to vary for each eddy in the following analyses.

While testing the application of the model to the observed data, it was noted that the fit of the model curve to the observed data could be improved by limiting the effects of the sharply declining end of the curve. (See Figure 13.) If one examines the colder end of the model curve, one notices that the model has a stagnation point at the downwelling region. That is, the velocity at the surface goes to zero, and the waters are allowed to cool infinitely. As discussed earlier, the model is not 
applicable to this region; the real surface temperature is not infinitely cooler. In order to reduce error caused by this singular point, the model curve is not applied to the pixel corresponding to the temperature minima. This gives the result of a better fit to the data (i.e. mean squared error square per pixel) and a method of eliminating a physical discrepancy. On the other hand, this also necessitates the removal of two-pixel eddies from the model application.

The analysis of the remaining (three-pixel and larger) eddies shows that the bulk temperature values for the smaller eddies show a larger variation than those of the larger eddies. It also appears that these smaller eddies tend to yield a lower bulk temperature estimate (on average) than the larger eddies. To reduce the effect of this possible bias, eddies smaller than five pixels have simply been removed in the final bulk temperature estimate (as defined by the spatial average of the remaining bulk temperatures). More details about the specifics of the application method are given in Appendix B, along with the MatLab code used in the application of the model to the observed data.

Lastly, some of the older data analyzed had obvious instrumentation errors. In one of the data sets analyzed, there were 'dead' pixels (i.e. pixels in the infrared camera's charge coupled device that failed to register any temperature), and in the two older sets, the image was grainy due to pixilation. Given that the algorithm developed applies the model to every line of data between a maximum and a minimum, higher levels of pixilation 


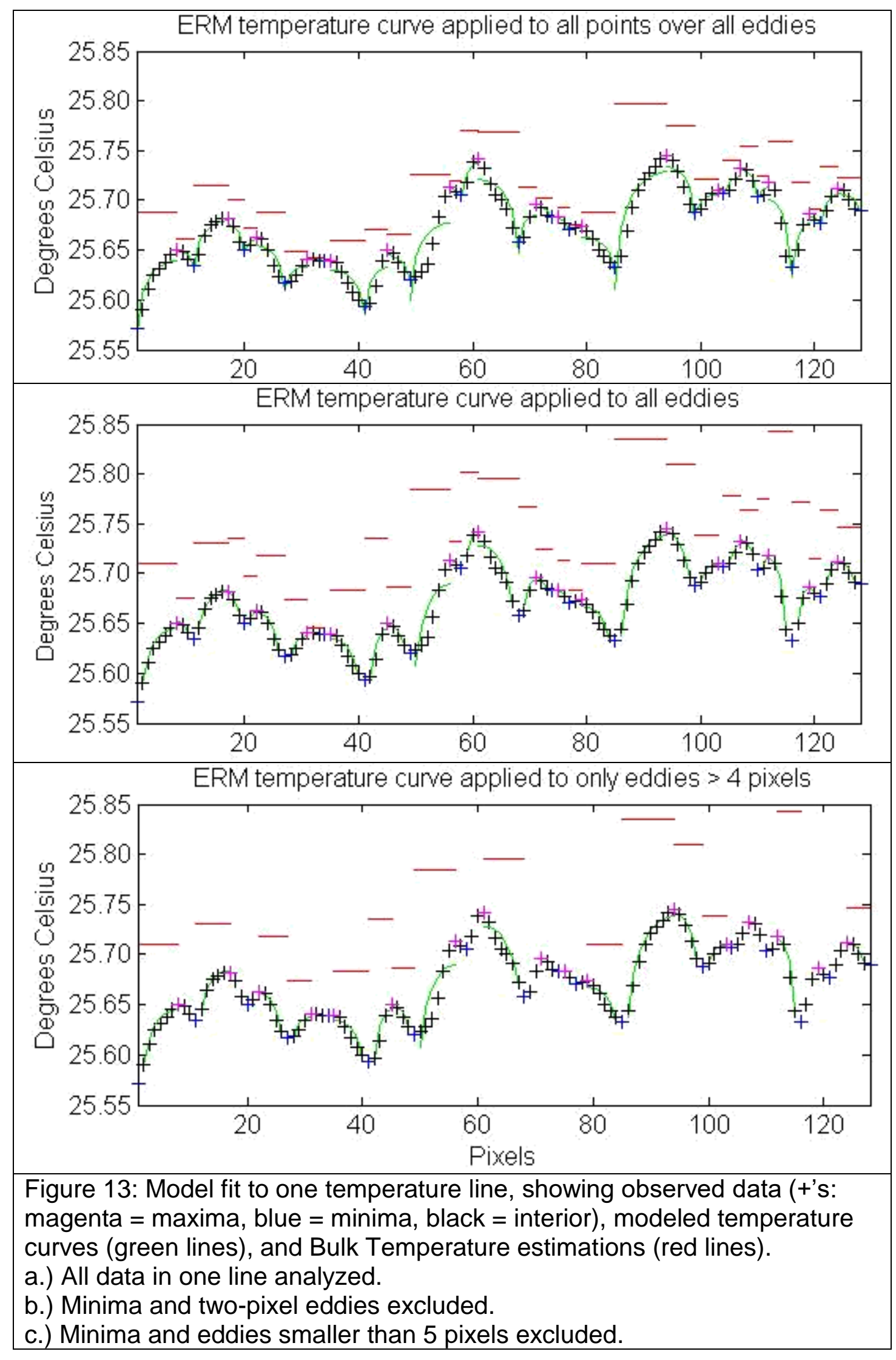




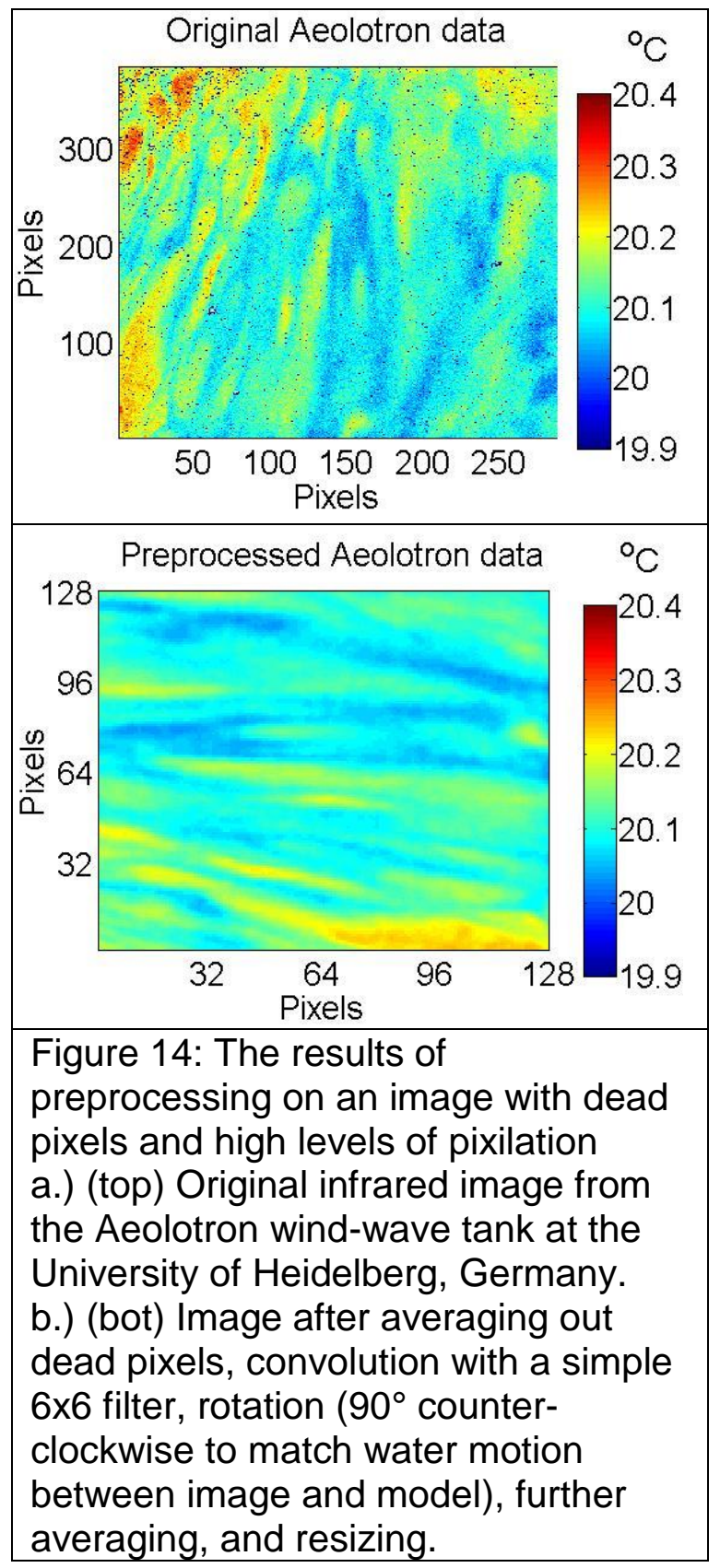

resulted in applying the model to noise in the image, and not to the underlying observed temperature patterns. In order to eliminate the dead pixel effect, those locations were simply filled with the average of values around them. The pixilation was eliminated by applying a simple $6 \times 6$ box filter with the image, and then further averaging the data by taking the mean of a $2 \times 2$ box of data. Thus, for two of the data sets, the matrix actually used in the analysis was less than half of the original image (taking into account the lost edges, where the convolution decreases the values). See

Figure 14 for an example of a pixilated image and the preprocessing.

To summarize the procedures, the following steps were taken to apply the Eddy Renewal Model to the observed data.

1.) Any necessary preprocessing is preformed to eliminate dead pixels and/or pixilation errors from older cameras. 
2.) The direction for line-analysis is selected perpendicular to the direction of mean current, which is assumed to be the wind direction.

3.) Each line of data is analyzed to determine the temperature maxima and minima. Each interval between temperature maximum and minimum (inclusive) is defined as one eddy.

4.) The model is applied to each eddy, excepting the temperature minimum pixel; any two-pixel eddies are not modeled.

5.) The model curve is divided into $(n-1 / 2)$ segments (where ' $n$ ' is the number of pixels in the observed eddy, including the maximum and minimum) and averaged for each segment; the $1 / 2$ segment is applied to the maximum point.

6.) The model is matched to the data by matching the temperature of the pixels to the mean temperature of the model curve segments.

7.) The bulk temperature is allowed to vary for each eddy and is determined by a least-squares best fit analysis between the model curve and the observed data.

8.) Any pixels not assigned a model value in this analysis (minima of all eddies and maxima of two-pixel eddies that are not shared with a larger eddy) are assigned the value of the original data point (for continuity's sake), but this value is not included in the error analysis.

The above algorithm is then applied over an entire image to obtain the modeled temperature and bulk temperature fields. The results of this analysis are given in the following section. 


\section{Section 2. Results of Image Analysis}

By applying the methods discussed in the previous section over an entire image (i.e. all lines of data), an entire modeled temperature field is generated, along with the associated bulk temperature field. This modeled temperature field can then be compared to the original image to determine the mean-squared error. (This error analysis was used to determine the best way to apply the model curve to the observed data, and to determine how the bulk temperature would be allowed to vary.) In total, more than 13,000 images were analyzed using this method. These images come from three different sources. The newest set of in situ data consists of images from the 2001 GasEx2001 experiments in the South Equatorial Pacific. These are contrasted with a set of lab tests done during wind-wave experiments in 2004 in the circular wind-wave tank called the "Aeolotron" at the University of Heidelberg, Germany. The earliest set comes from the CoOP experiments in 1997 in the northern Atlantic. Thus, the model has been applied to images taken using three different camera equipment set-ups in a variety of wind and wave conditions.

The basic form of the results is a matrix, equal in size to the image analyzed, of modeled temperature values and a matrix (of equal size) of bulk temperature values. Two bulk temperature estimates from the Eddy Renewal Model are obtained: the estimate from all eddies (3 pixels and larger), and the estimate from eddies 5 pixels and larger. Next, the mean bulk temperature 
estimate for each image is obtained using the size of the eddies to weight the mean. The size of each eddy is defined as (n-1) pixels, where $n$ is the number of pixels from maximum to minimum, inclusive.

If the analysis is completed over a series of images, the resulting bulk temperature estimate (spatial average) can be viewed as a time series of these spatial averages. This can be directly compared with the bulk temperature estimate based on the Surface Renewal Model (Garbe, et. al., 2004), which is also spatially averaged.

For the GasEx2001 data, the results of the SRM analysis are shown as a red line, while the two ERM analysis results are shown as a blue line (all eddies) and a magenta line (five pixels and larger). The SRM results have been smoothed; whereas the ERM results have not been. The sharp peaks that appear throughout ERM bulk temperature estimates in the time series Figures correlate with high levels of infrared reflectance, as observed when watching the original films. The wind-wave tank and CoOP data time-series do not have the results from the SRM analysis for comparison.

The sample images shown below demonstrate different conditions observed in the data. Figure $15 \mathrm{a}$ is a sample image with little or no breaking activity, and little or no reflected infrared interference. (Figures 15b, 15c, and $15 d$ are the resulting analysis of $15 a$.) Figures 16 and 17 show the influence of breaking events. (The former highlights the effects of an active breaking wave in the image, while the latter shows the residual turbulent eddy field after a breaking wave has passed the area.) Figure 18 shows the effects of 
infrared interference on the image. In the analysis of the time series figures, in conjunction with viewing the videos, it seems that breaking events appear on the time series as the regions of gentle increase and decrease in temperature, while the IR interference is easily detectable in the time series as sharply increasing then decreasing temperature change. Figures 15, 16, 17, and 18 are all from the GasEx2001 experiments. Two images from the Aeolotron experiments have been included to show the different patterns that may be observed in a wind-wave tank. Figure 19 shows more streaky temperature patterns, while Figure 20 shows patterns more similar to what were seen in the in situ data. Although data from the Co-OP 1997 experiments were analyzed, they are not included in this section. (The timeseries figures for the Co-OP data may be found in Appendix A, along with all of the time-series plots generated in this research.) 


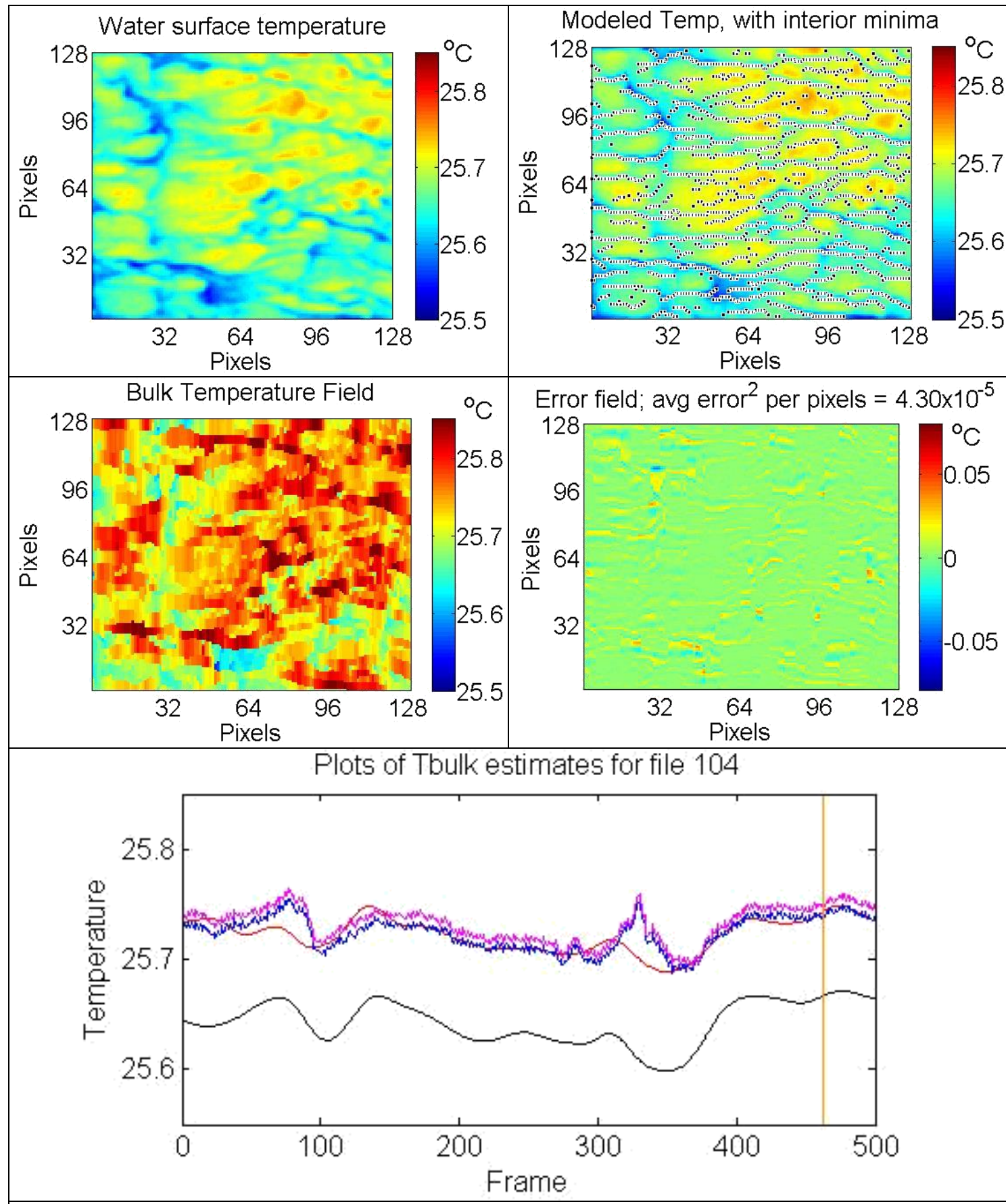

Figure 15: Image analysis of a "clean" frame.

a.) (top left) Original infrared image of the sea surface. (Frame 462 of this file)

b.) (top right) Modeled temperature field. (Dots are local minima.)

c.) (mid left) Modeled bulk temperature field.

d.) (mid right) Error-field.

e.) (bottom) Time series analysis showing mean surface temperature (black); mean bulk temperature from all eddies (blue); mean bulk temperature for only eddies larger than 4 pixels (magenta), and bulk temperature estimate from the Surface Renewal Model (red). The orange line shows location of this frame. 


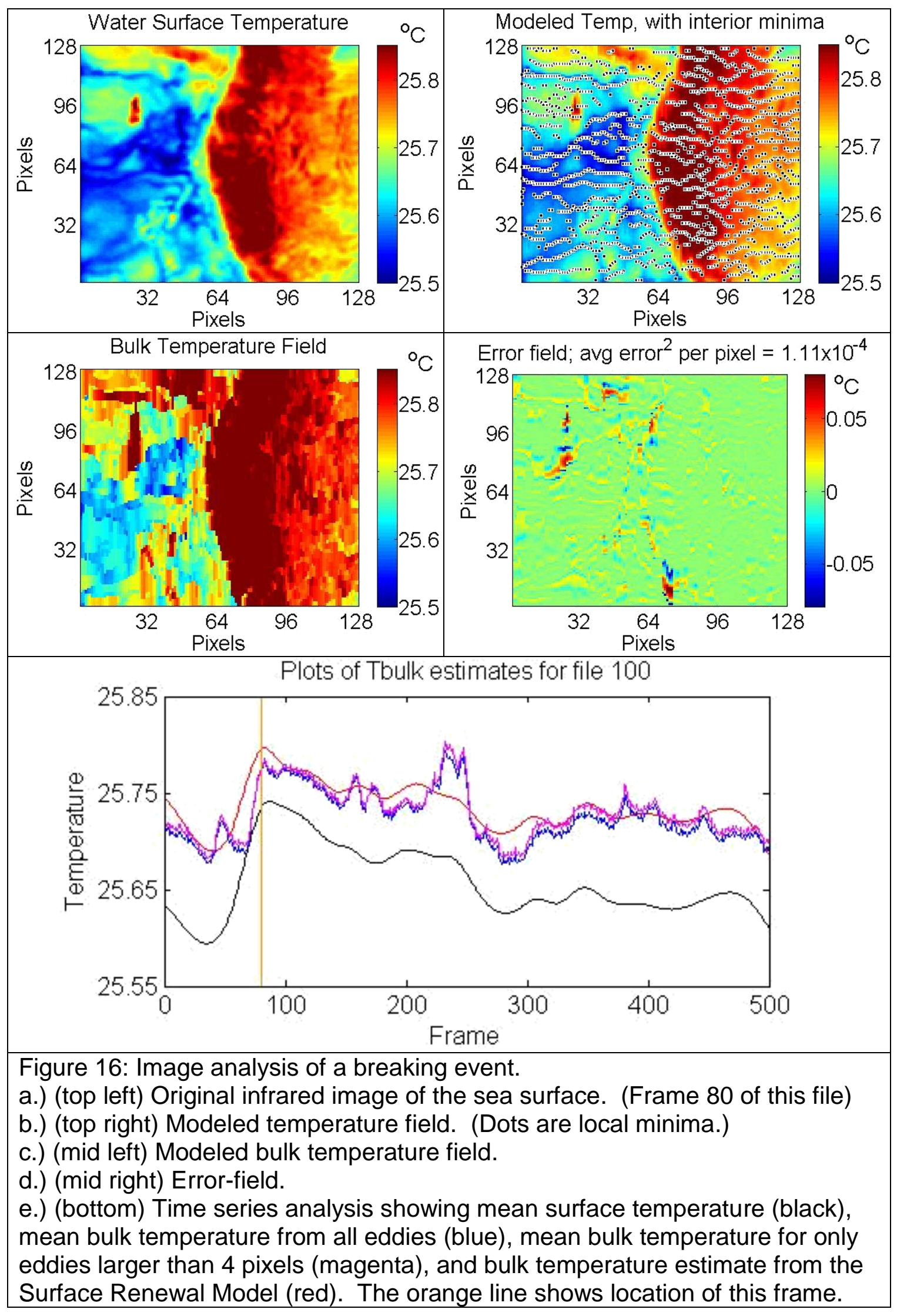



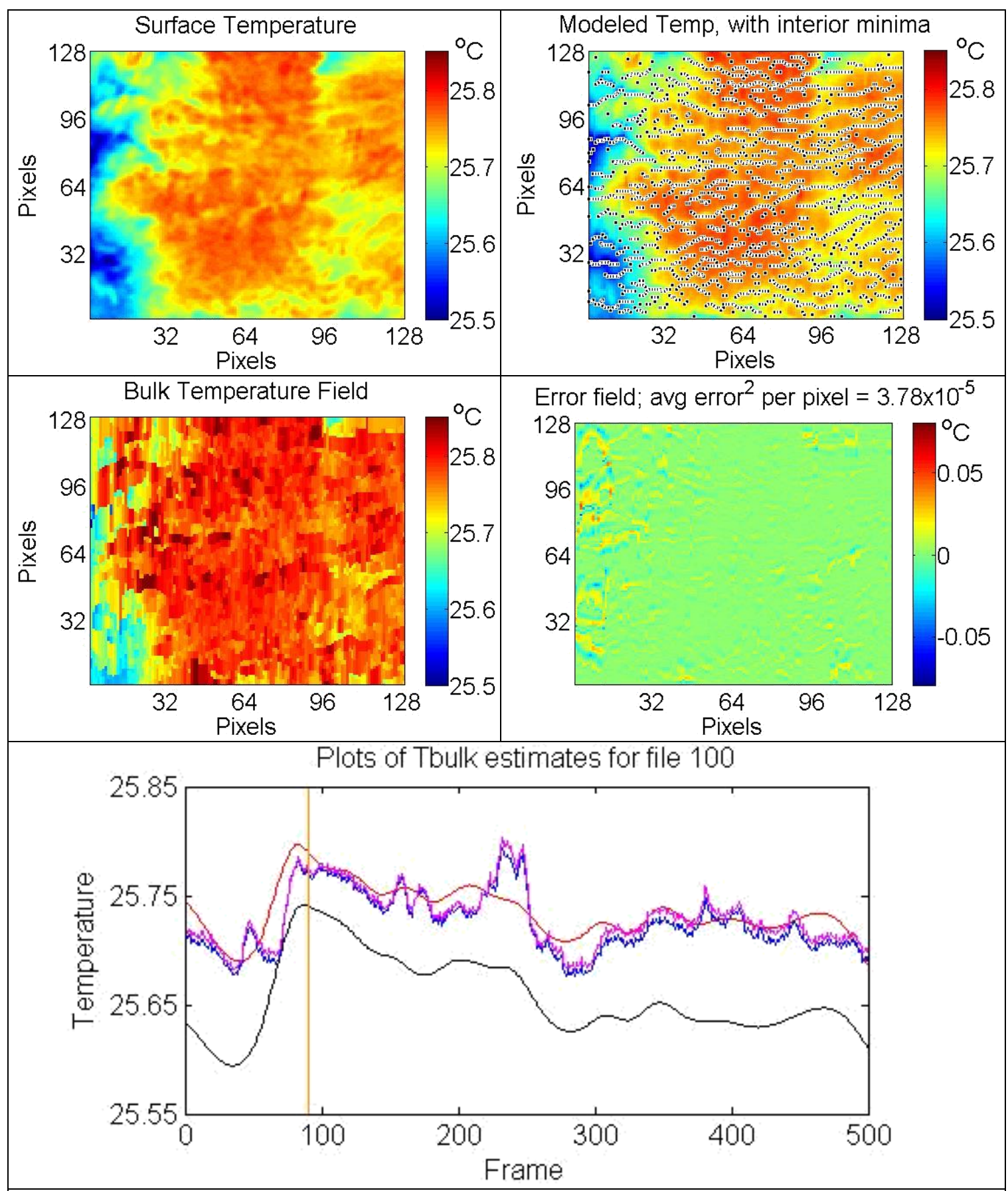

Figure 17: Image analysis after a breaking wave has lost much of its energy to turbulent mixing. This is $1 / 10^{\text {th }}$ of a second after the previous figure

a.) (top left) Original infrared image.

b.) (top right) Modeled temperature field with internal minima.

c.) (mid left) Associated temperature field.

d.) (mid right) Error field.

e.) (bottom) Time series analysis, with the orange line showing location of this frame in the series (90). 

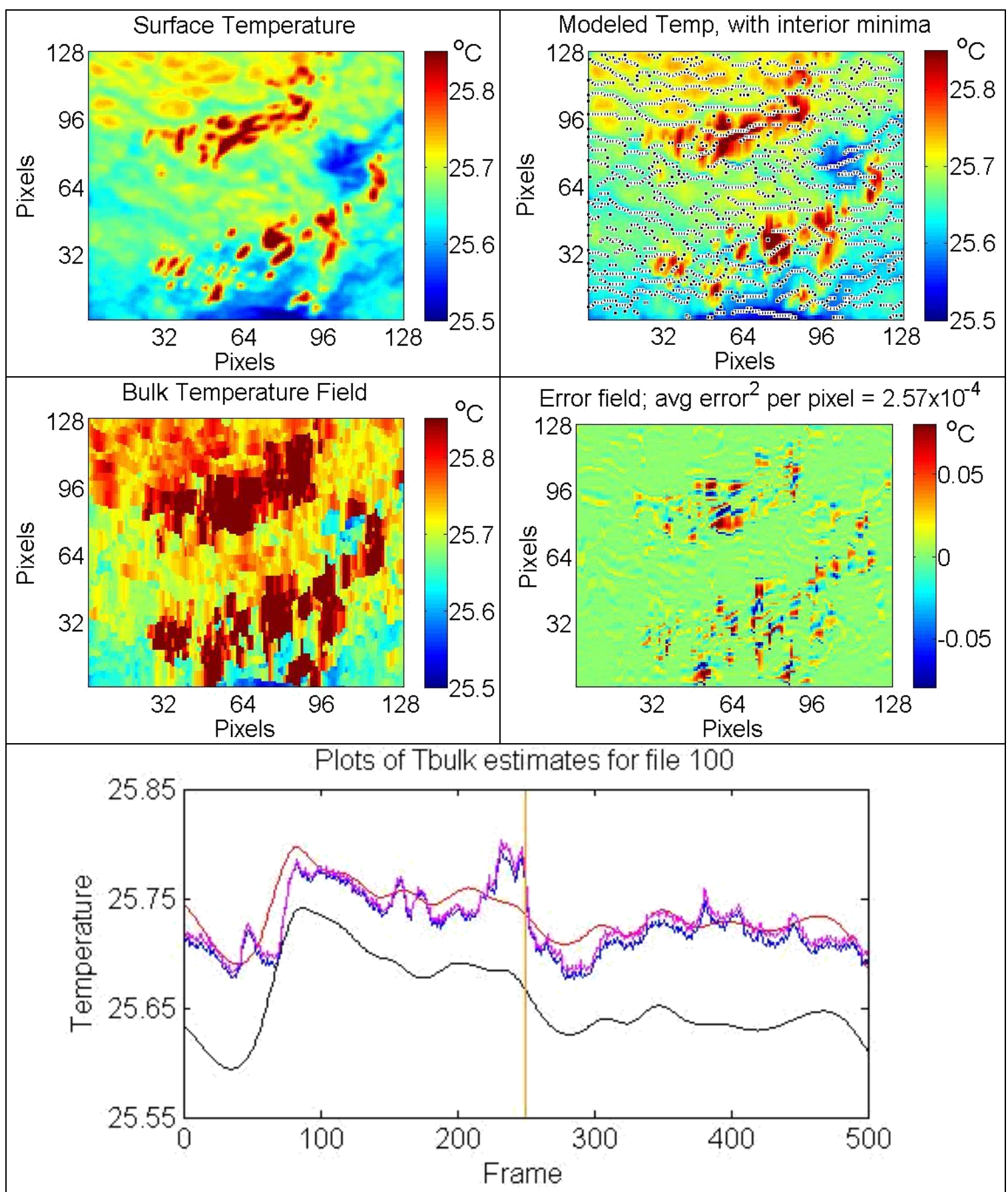

Figure 18: Image analysis with higher levels of reflected infrared.

a.) (top left) Original infrared image.

b.) (top right) Modeled temperature field with internal minima,

c.) (mid left) Associated temperature field.

d.) (mid right) Error field; notice the higher levels of error corresponding with the same location as the unusually high temperatures in the original image. e.) (bottom) Time series analysis, with the orange line showing location of this frame in the series (250). 


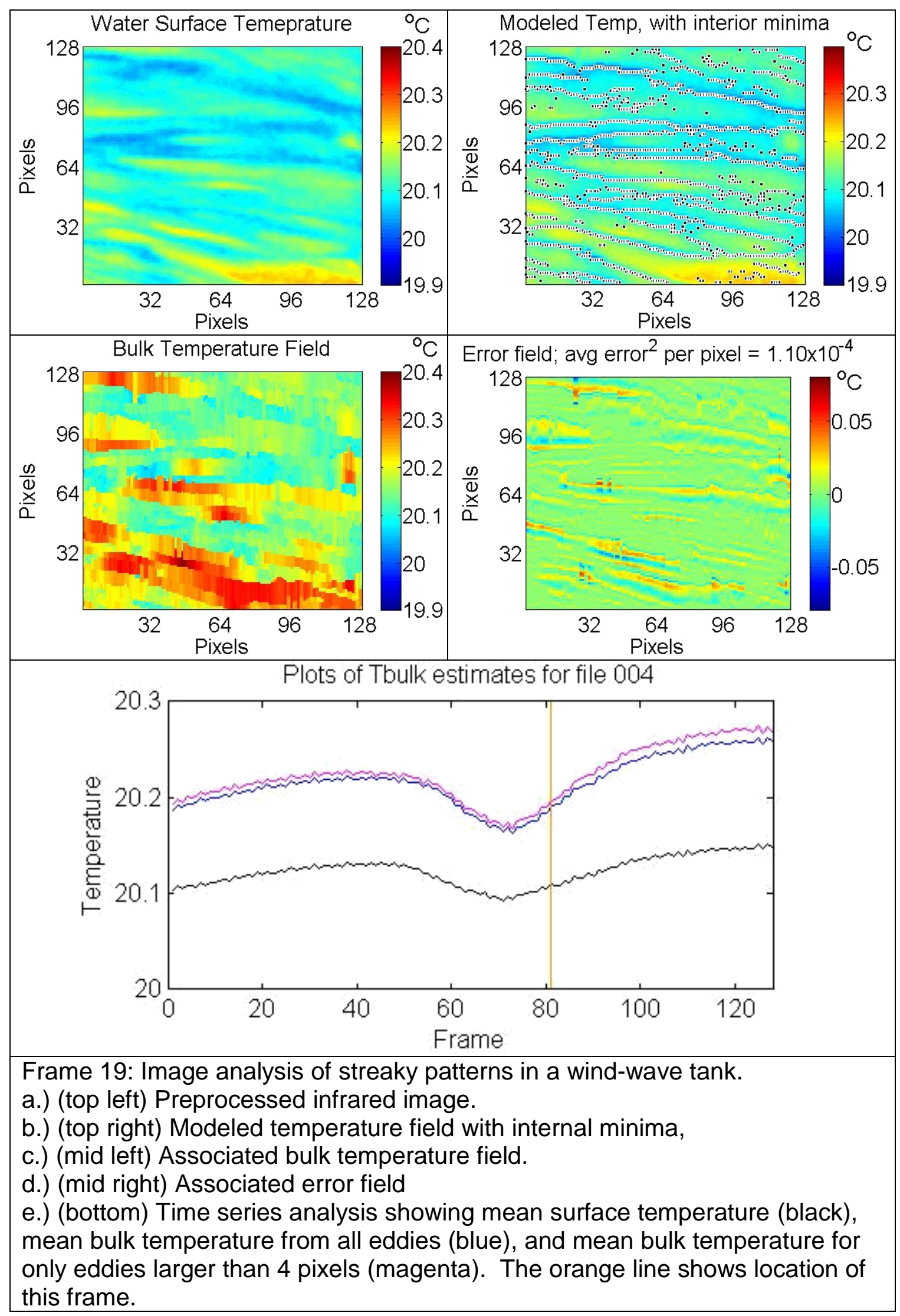




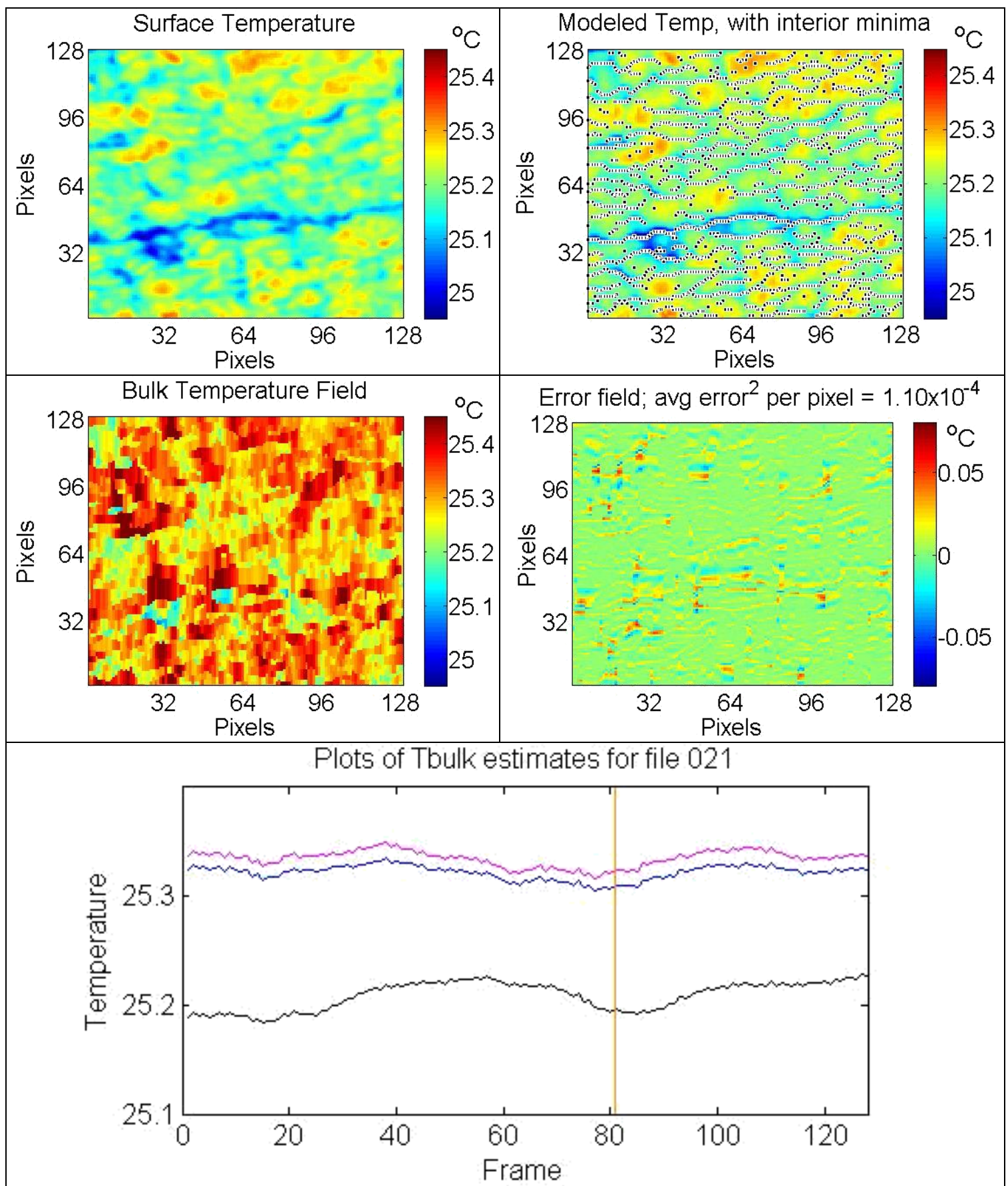

Figure 20: Image analysis of a frame from a wind-wave tank showing patterns more like those from in situ images.

a.) (top left) Preprocessed infrared image

b.) (top right) Modeled temperature field with internal minima

c.) (mid left) Associated bulk temperature field

d.) (mid right) Associated error field

e.) (bottom) Time series analysis showing mean surface temperature (black), bulk temperature from all eddies (blue), and bulk temperature for only eddies larger than 4 pixels (magenta). The orange line shows location of this frame. 


\section{Section 3. Discussion of Image Analysis Results}

In general, the Eddy Renewal Model is able to reproduce the surface temperature field quite well. In the images presented in the previous section, the average total squared error is on the order of $0.5\left({ }^{\circ} \mathrm{C}\right)^{2}$ for the entire image, with a mean error square on the order of $5 \times 10^{-5}\left({ }^{\circ} \mathrm{C}\right)^{2}$ for images free of reflected infrared. This suggests that the general shape of the surface temperature curve, as derived in section two of chapter three, is a good description of the real temperature distribution in the eddies observed at the sea-surface. The one notable exception is the colder end of the model, for reasons that have already been discussed.

It is also evident that the agreement between the modeled and observed temperature profile is generally poor when the image suffers from incidents of infrared reflectance. In the error field in Figure 18d, higher error values show up in the regions with IR reflectance. These same locations also correspond to much higher bulk temperature estimates. In the time series in Figure 18e, IR reflectance shows up as periods of unusually high bulk temperature estimates. In contrast, Figures 15, 16, 17, 19, and 20 all show very limited (or no) levels of IR reflectance. These observations, suggest that it might be possible to use a certain threshold of the mean squared error to detect, and possibly eliminate, incidents of infrared reflectance.

The SRM uses a statistical method of analysis based on intermittent surface renewal events, such as breaking events, whereas the ERM is based 
on the physics of small-scale stationary turbulent eddies. Therefore it is natural to question the new model's ability to adequately describe the temperature field at and behind a breaking event. Figures 16 and 17 are images taken during the same breaking event. In the first image, the edge of the breaking event is clearly visible (as the large crescent of warmer waters). In Figure 17 (which was taken one tenth of a second later than Figure 16), the breaking event has disintegrated into intense small-scale turbulent eddies. In examining the error field, it seems that the new model is able to describe the temperature during and after breaking events quite well. This would make sense if the breaking event has generated small scale turbulent eddies that quickly become quasi-stationary, and if the surface renewal is mainly controlled by such eddies rather than the instantaneous passing of the breaking front itself.

To quantify the accuracy of the fit of the model curve to the observed data, error analyses are included with each analyzed image. Furthermore, in order to see the average shape of the eddy temperature curve, it is useful to take a large number of observed eddy temperature curves, nondimensionalize them, and average them so that the mean "basic shape" curve is compared against the Eddy Renewal Model temperature curve. In order to non-dimensionalize the eddies, one needs to remove the characteristics of length, temperature spread, and highest temperature value. This can be done by first subtracting the calculated bulk temperature from the observed temperature and then dividing the results by the eddy intensity. This analysis 
is done separately for each eddy size, so that the ERM model applicability is examined as a function of the eddy size. (See Figure 21.)

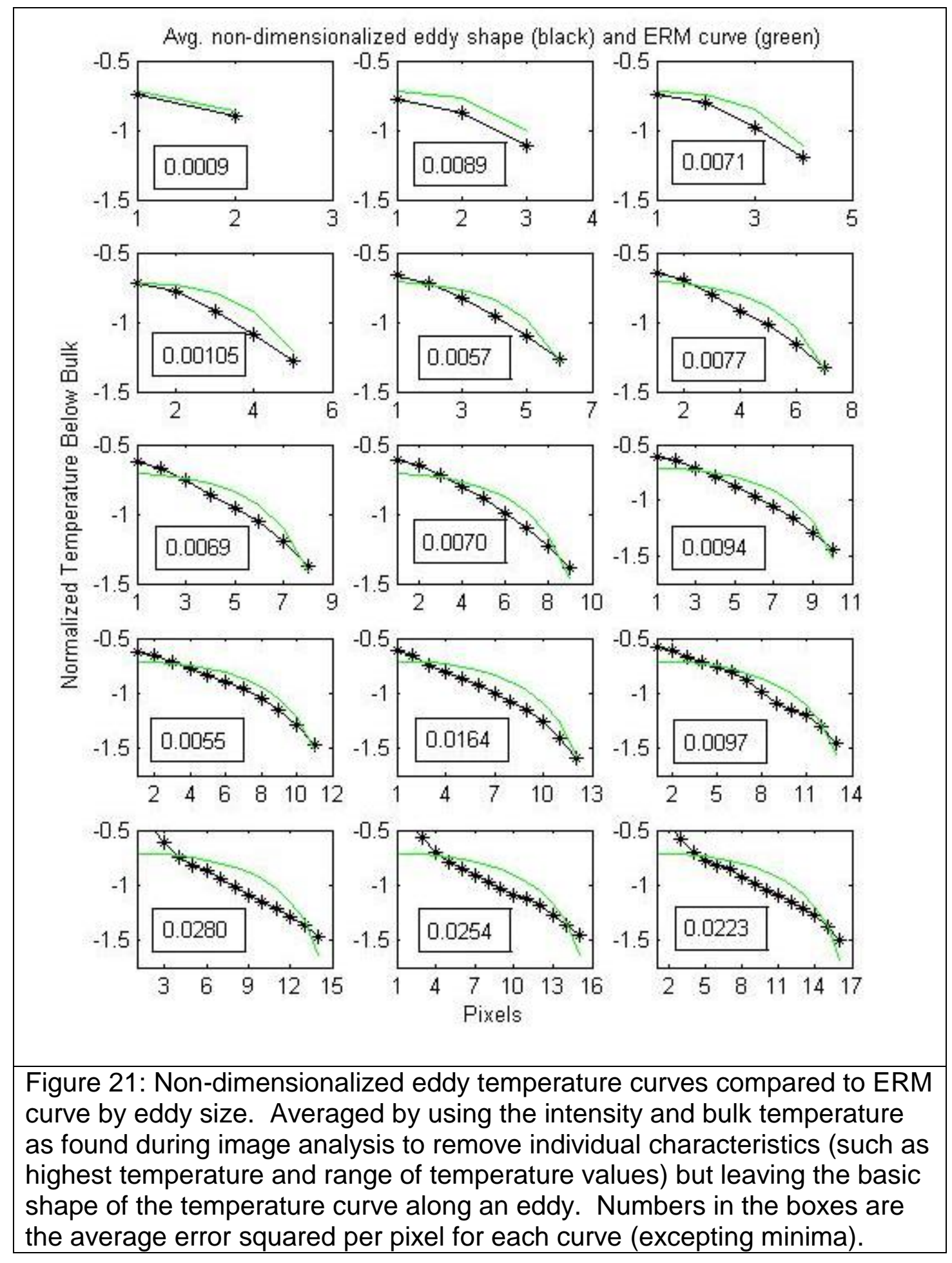


For eddy sizes 5 through 11 or 12 pixels long, it would seem that the ERM curve is a good basic fit (except at the minima, as noted earlier). The smaller eddies, although still a good fit, as defined by the error squared values in each plot, were found to have too much variation in their bulk temperature estimates. It has always been assumed that the largest eddies would show a better fit to the model curve, as they would have less area that could be effected by the horizontal temperature gradients near the downwelling, however, analysis of the basic curve fit suggests that this may not be so. The three largest eddy cases (in Figure 21) show that the observed temperature curve has positive curvature near the maximum, resembling the model curve of the Surface Renewal Model. It is possible that the quasistationary assumption may not be applicable for very large eddies.

Even though the data curves in Figure 21 show a good basic fit to the model, it is possible that eddies with a poor fit are distorting the shape of the mean curves. This could be easily shown if a large percentage of eddies have an error squared per pixel (ESPP) smaller than the mean value. Figure 22 shows that this is the case for the smaller eddies. Using only eddies with an ESPP smaller than

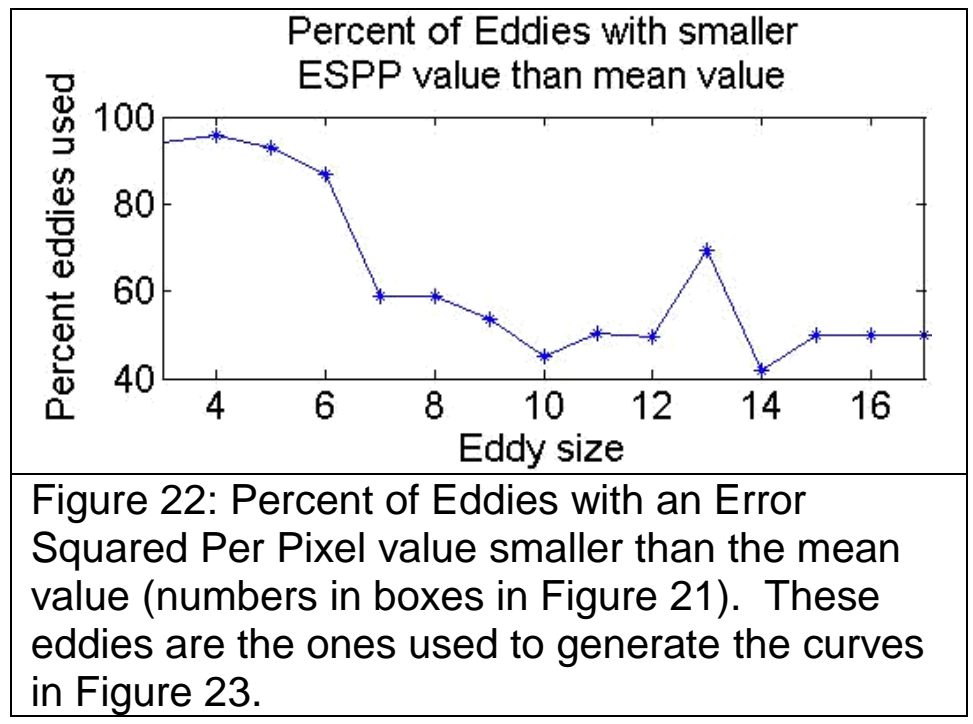


the mean, the mean temperature curves are again found (Figure 23). This plot confirms that the basic shape of the eddy renewal model curve is a good match for the majority of the observed eddies.

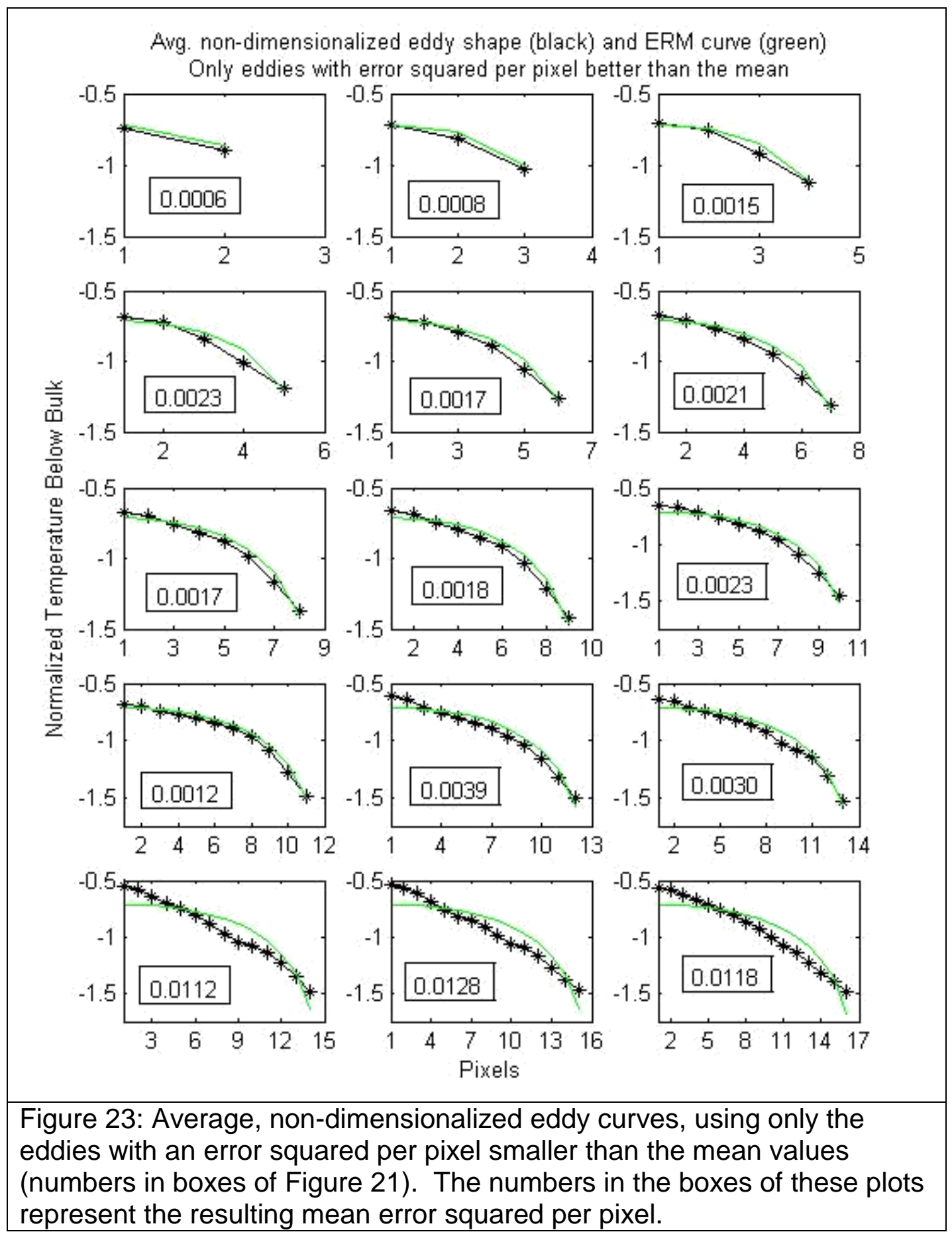


In summary, the Eddy Renewal Model is able to accurately model the sea surface temperature variations due to near surface turbulence. The application of the model to data taken in a wind-wave tank generally yields very similar results. In Figure 20 the surface temperature pattern is very similar to the GasEx2001 data. In Figure 19, the surface temperature patterns show larger and more elongated eddy patterns. In both cases, the ERM reproduces the temperature patterns equally well. 


\section{Section 4. Statistics Obtained From Image Analysis Results}

In order to apply the modeled temperature curve to the observed data, only two variables were used: the bulk temperature and the "intensity" of the eddy. The "intensity" comes from equation [2.3.7] and is defined as $\delta j o / k$ which is proportional to the difference between the bulk temperature and the mean temperature of the curve. Thus, once the normalized temperature solution was obtained, this curve could be applied to the observed data by finding the bulk temperature and intensity with the least mean squared error.

Given that the $\delta$ is proportional to the square root of $1 / \tilde{k} u_{0}$, (see equation [2.3.9]) the intensity $\delta j o / K$ is directly related to the surface divergence if the heat flux is assumed to be constant over the image. It is, therefore, of interest to examine whether a high correlation exists between the eddy size and eddy intensity. As seen in Figure 24a, there does not appear to be such a correlation between eddy size and intensity. Neither does there appear to be a correlation between the size of the eddy and the bulk temperature estimate for that eddy (Figure 24b). Instead, the variance of the estimates increases as the eddy size decreases. This suggests that the individual estimate of the bulk temperature or the eddy intensity is less accurate for smaller eddies (as expected), but the mean estimates are robust and are not influenced by the eddy size. This also suggests that the overall variation of the bulk temperature and intensity estimates is overestimated based on our method; part of the variation is likely due to the inaccurate model application 
to smaller eddies. In fact, Figure $26 c$ and $26 d$ show that the variability of the bulk temperature is larger than that of the surface temperature. However, it is likely that the true variation of the bulk temperature is less than that of the surface temperature. The variation of the intensity in Figure $26 \mathrm{~b}$ is also most likely overestimated.

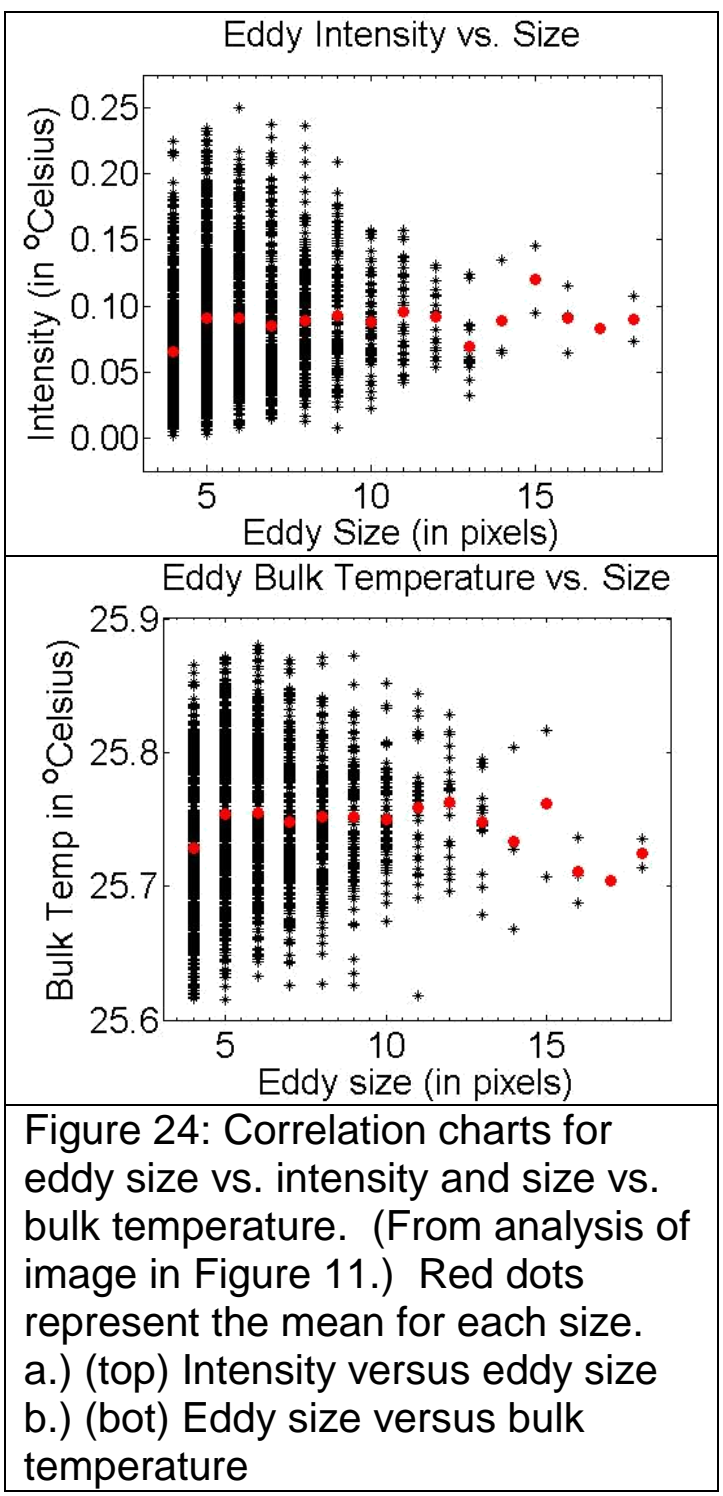

Figure 25 shows the overall correlation between the eddy intensity and the bulk temperature, with colors indicating different eddy sizes. Clearly the eddy intensity is correlated to the bulk temperature. However, given how the intensity was defined in the computation (as a function of the difference between the bulk temperature and the mean modeled surface temperature), the apparent correlation along a line with a positive slope is most likely an artifact of the computational analysis. That is, a bulk temperature value that is artificially too high will produce an intensity value that is also artificially too high. However, for a given bulk temperature, the distribution of the eddy intensity likely represents the true 
variability of the eddy intensity. Furthermore, this variability seems to be independent of the eddy size. (The data points along the bulk temperature value of $25.7599^{\circ} \mathrm{C}$ are an artifact from computation; this value is the highest observed surface temperature for this frame.)

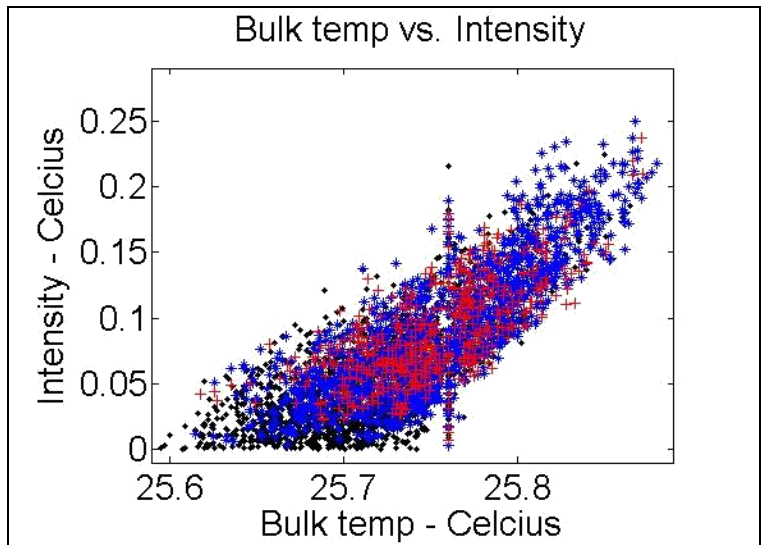

Figure 25: Bulk temperature vs. eddy intensity. (Analysis of same image as in Figure 11) Black dots are from eddies sized 3 or 4 pixels; blue stars are from eddies 5,6 , or 7 pixels; red crosses are from eddies 8 pixels and larger.
Throughout the previous discussion, the strength of each eddy was defined as the "intensity." Given that the intensity is defined as $\delta j o / K$, and $\delta$ is defined as in equation [2.3.9], if the eddy size is found by image analysis, and one assumes that all other values are constant (which is a good assumption for specific heat, density, and

conductivity, but may not be for surface flux), one can then solve for $u_{0}$. The assumption of a constant surface flux is almost certainly false; however, for the purposes of examining the variation of $u_{0}$, this assumption may be reasonable. Given an average surface flux of $100 \mathrm{~W} \mathrm{~m}^{-2}$, and typical values for specific heat, density, and conductivity of water $\left(4181.3 \mathrm{~J} \mathrm{~kg}^{-1} \mathrm{~K}^{-1}, 1030 \mathrm{~kg}\right.$ $\mathrm{m}^{-3}$, and $0.6 \mathrm{~W} \mathrm{~m}^{-1} \mathrm{~K}^{-1}$, respectively), the distribution of surface velocity maxima for the observed eddies is seen in Figure 27a. Of particular interest is the difference in distributions for different eddy sizes (Figure 27b). This figure suggests that larger eddies have larger values of $u_{0}$. 

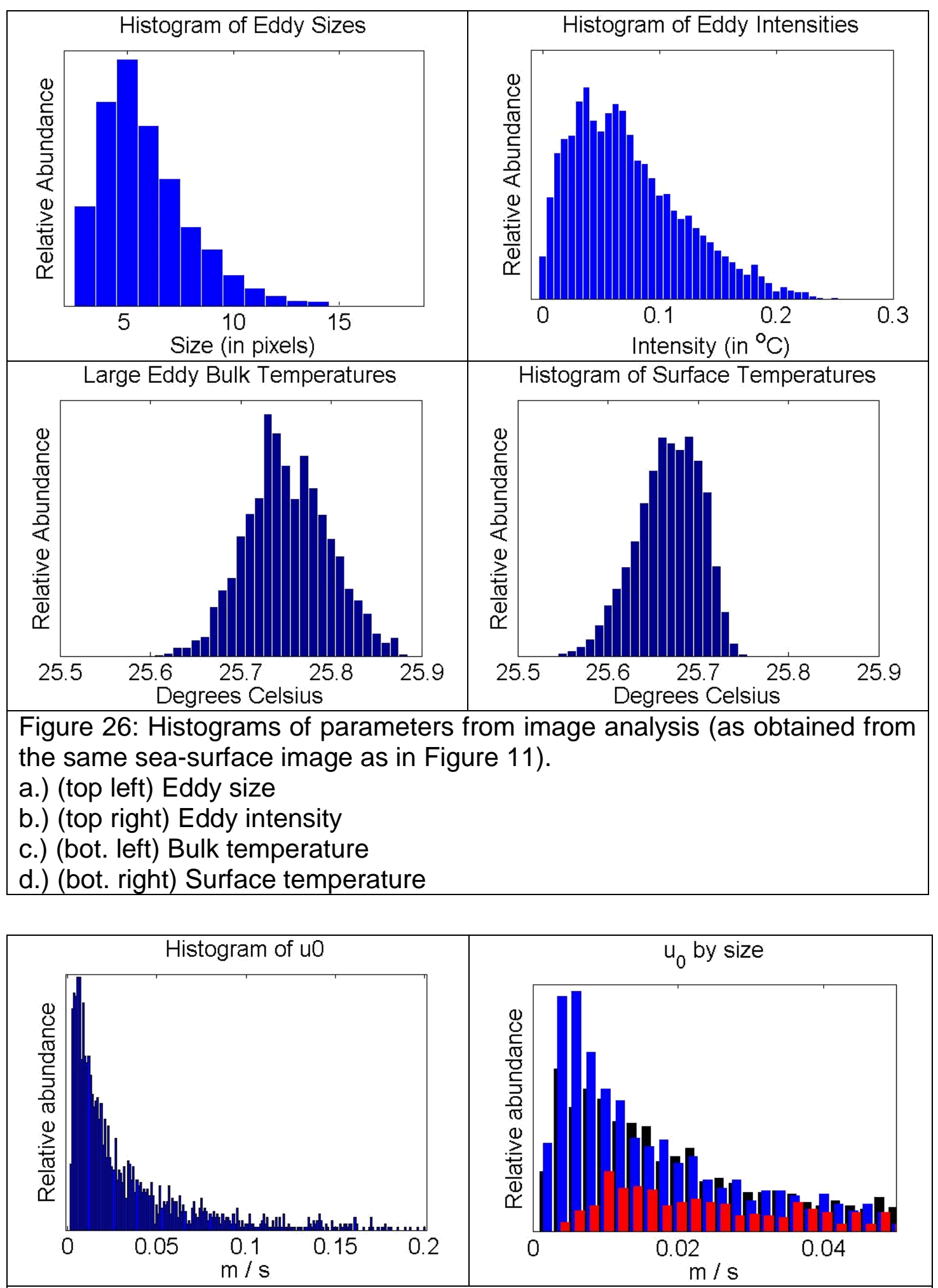

Figure 27: Distribution of surface divergence maximum velocities. (Analysis from same image as in Figure 11)

a.) (left) uo for all eddies (3 pixels and larger)

b.) (right) uo according to size of eddy. (Color as in Figure 25) 


\section{Chapter 5 Conclusion}

The proposed analysis of infrared images based on the Eddy Renewal Model is a novel approach to deducing the bulk temperature by analyzing the temperature gradients found at the air-water interface. Whereas the previous approach based on the Surface Renewal Model uses a statistical distribution of the surface temperature to derive the bulk temperature, the new approach uses direct application of a modeled temperature curve to the observed data to obtain local estimates of the bulk temperature. The application of the ERM to images of the air-water interface (both in situ and in a wind-wave tank) shows that the basic shape of the model is a good first-order approximation of the temperature distributions found in these small-scale turbulent eddies.

One clear advantage of the ERM is that it explicitly incorporates the water motions of individual eddies. Although the ERM is not a fully threedimensional model; as a first order approximation, the ability of the model to estimate the temperature variation below the surface is novel. One way to verify (or disprove) this model's applicability would be to make use of a device similar to the one currently being used to measure small-scale vertical temperature gradients near the sea surface (Ward, 2005).

There are benefits and disadvantages to each model. One of the major disadvantages of the ERM is the long data processing time required to analyze each image. Although more study is needed to fully understand all of the implications from these results, it would appear that both methods 
complement each other and are ultimately useful in bulk temperature estimations.

In the future, the ERM could be improved in different directions. First, by incorporating a more fully-three dimensional eddy field, the water motions could be better described. Second, it is of interest to quantify the relative importance of the time derivative term and the advection term in the heat equation. (While the SRM only considers the former, the ERM includes the latter only.) And lastly, given the results from this analysis, it might be possible to apply this model to better explain the gas exchange processes at the air-water interface. The impact of ERM on the air-sea gas transfer velocity estimates has not been fully explored. Previous works have used heat as a proxy tracer for the exchange of gases. The validity of this practice has been called into question for a variety of reasons, including the differences in surface conditions (constant surface gas concentration versus constant surface heat flux). Given the new model of water motions presented in this document, one next logical step would be to rerun the calculations for a constant surface concentration, given the subsurface motions calculated in the temperature / heat flux analysis, and compare this result to the previous model results. Although any of the former are excellent directions for future research, this last one could be of most use to the researchers who are trying to understand the interactions between the water and air and their implications for climatological models. 
Appendix A. Instrumentation Specifications and Experiment Details

The data presented in this thesis comes from three distinct experiments. The laboratory data comes from the University of Heidelberg's circular wind-wave tank the Aeolotron. The data was collected during August of 2004 by the staff at that facility. The in situ data analyzed in the previous sections comes from the GasExll experiment performed at sea (in the Southern Equatorial Pacific ocean) in 2001. Also included (in Appendix B) is the analysis of data obtained during the CoOP1997 cruises in the Northern Atlantic ocean. These data sets were taken using different equipment and represent the ocean surface under different conditions. The following tables show the basic differences between the instrumentation used and the physical conditions during each experiment.

Table A1-1: Instrumentation Specifications

\begin{tabular}{|l|l|l|l|l|}
\hline Data Set & Camera Type & Spectral Range & NeDT & Frame Rate \\
\hline GasExll & Amber Galileo & $3-5 \mu \mathrm{m}$ & $\sim 25 \mathrm{mK}$ & $100 \mathrm{~Hz}$ \\
\hline AELOTRON & $\begin{array}{l}\text { Thermosensorik } \\
\text { CMT 384 M }\end{array}$ & $3-5 \mu \mathrm{m}$ & $<20 \mathrm{mK}$ & $130 \mathrm{~Hz}$ \\
\hline CoOP1997 & $\begin{array}{l}\text { Amber } \\
\text { Radiance I }\end{array}$ & $3-5 \mu \mathrm{m}$ & $\sim 15 \mathrm{mK}$ & $\begin{array}{l}1 \mathrm{~Hz} \text { for } 8 \\
\text { images, } \\
\text { followed by } \\
\text { a pause of } \\
52 \text { seconds }\end{array}$ \\
\hline
\end{tabular}


Table A1-2: Experiment Details

\begin{tabular}{|l|l|l|l|}
\hline Data Set & File & $\begin{array}{l}\text { Approximate Surface } \\
\text { Temperature Range }\left({ }^{\circ} \mathrm{C}\right)\end{array}$ & Wind Speed $(\mathrm{m} / \mathrm{s})$ \\
\hline GasExII 2001 & All & $\begin{array}{l}25.50-25.70 \\
(\text { Typical range: } 0.1)\end{array}$ & 5.1 \\
\hline Aeolotron & $001-003$ & $20.48-20.49$ & 5.0 \\
\hline & $004-005$ & $20.10-20.15$ & 5.0 \\
\hline & $011-013$ & $25.95-25.98$ & 5.0 \\
\hline & 014 & $26.24-26.26$ & 5.0 \\
\hline & 015 & $26.12-26.15$ & 5.0 \\
\hline & 016 & $25.93-25.96$ & 5.0 \\
\hline & $017-019$ & $25.70-25.72$ & 5.0 \\
\hline & $020-021$ & $25.17-25.23$ & 5.0 \\
\hline CoOP 1997 & 022 & $25.12-25.18$ & 5.0 \\
\hline & 201 & $12.36-12.63$ & 1.3 \\
\hline & 202 & $25.35-25.45$ & 10 \\
\hline & 203 & $20.39-20.79$ & 5.1 \\
\hline
\end{tabular}


Appendix B. Additional Figures and Results

GasEx2001 Results - Time Series Plots

The following are the results from the image analysis for the data from GasEx2001 experiments in the equatorial Pacific waters in 2001. Each Figure is a time series of the final results for the images in that file. Each time-series is 500 frames long, which corresponds to 5 seconds. These images have also been analyzed with the Surface Renewal Model to compare the two models. The bulk temperature results from the SRM analysis are in red. The blue line represents the Eddy Renewal Model bulk temperature estimate of all eddies (three pixels and larger), while the magenta line is the bulk temperature estimate from only eddies five pixels and larger. The black line is the mean surface temperature.

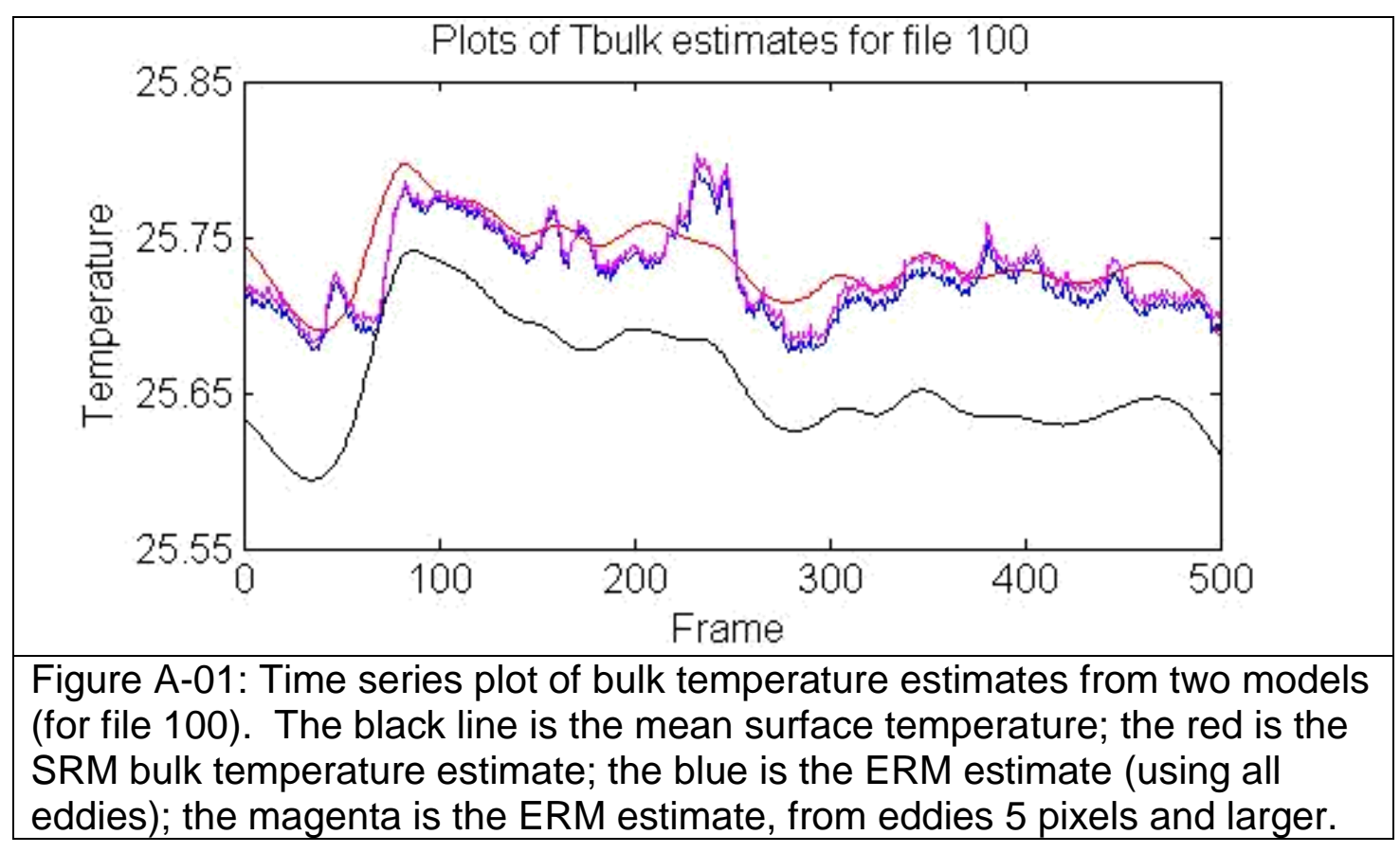




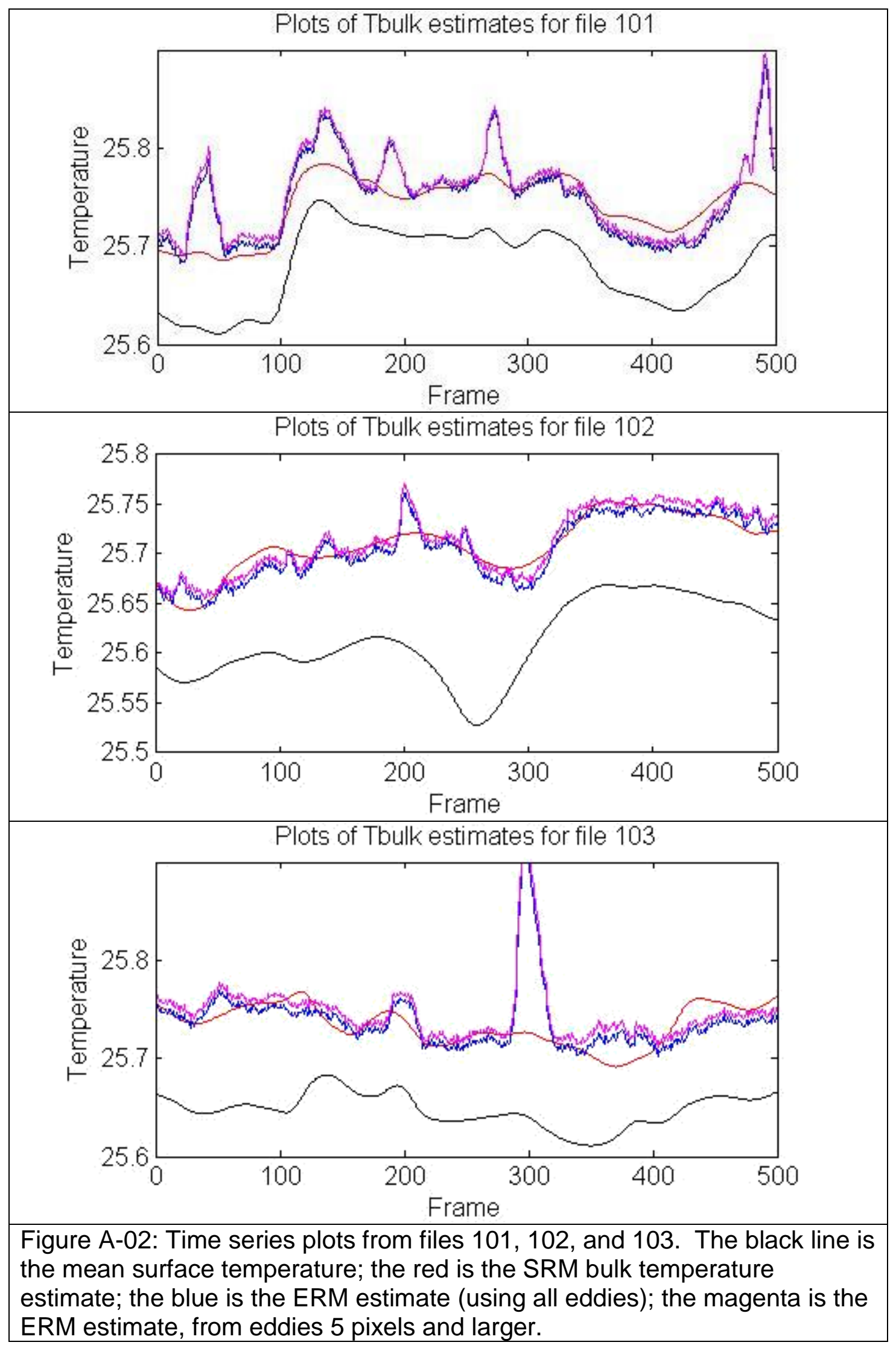




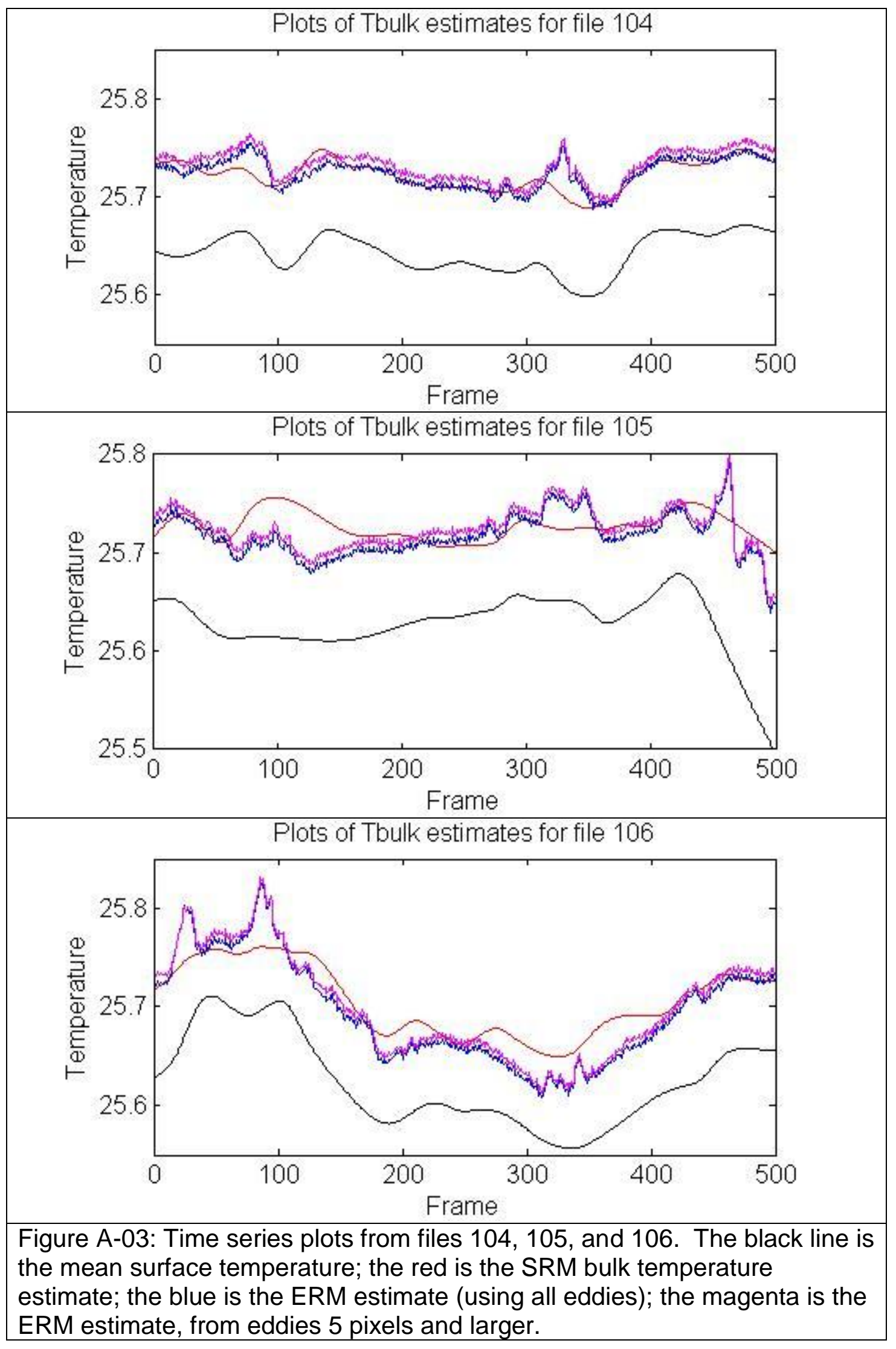




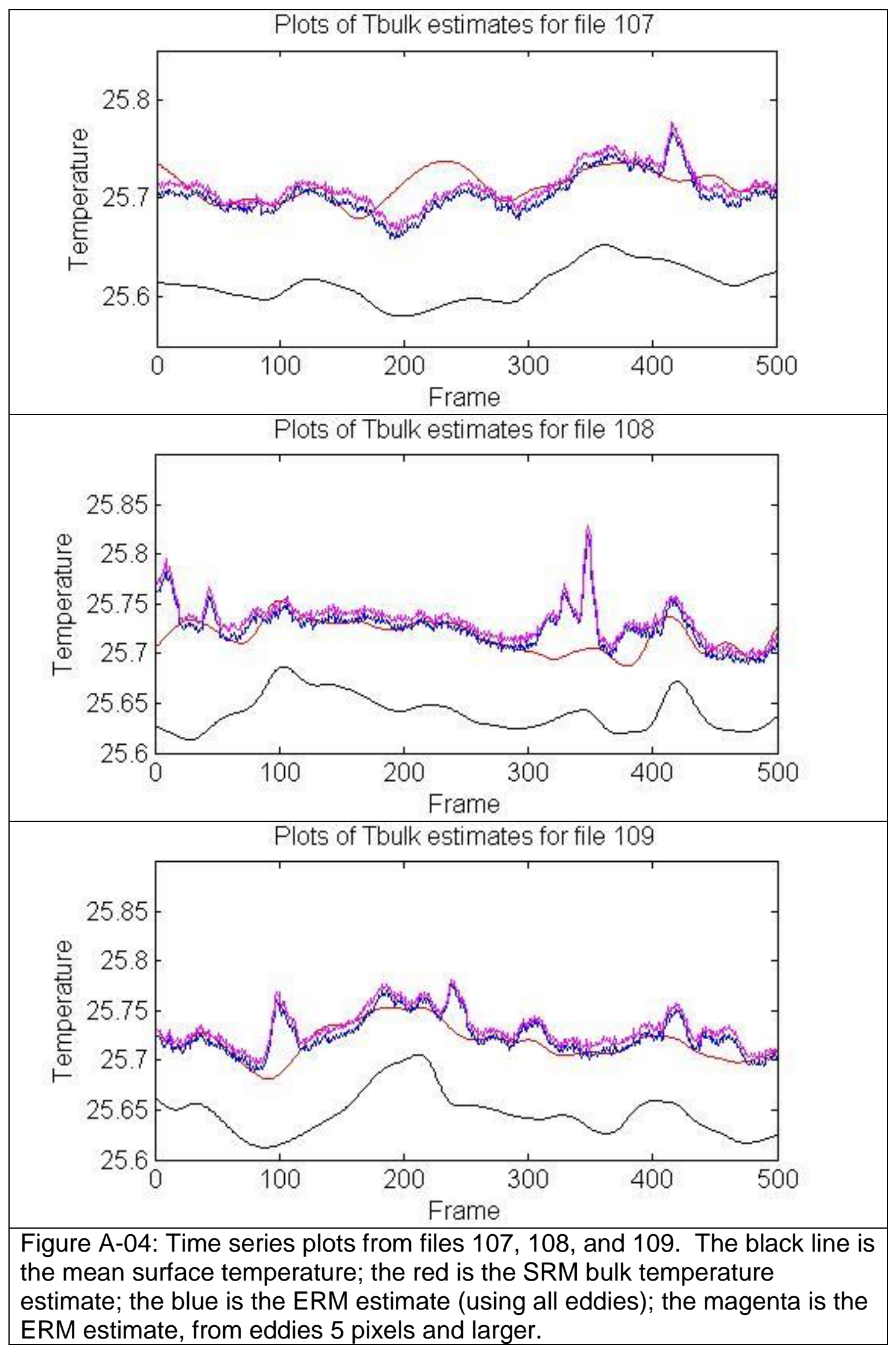




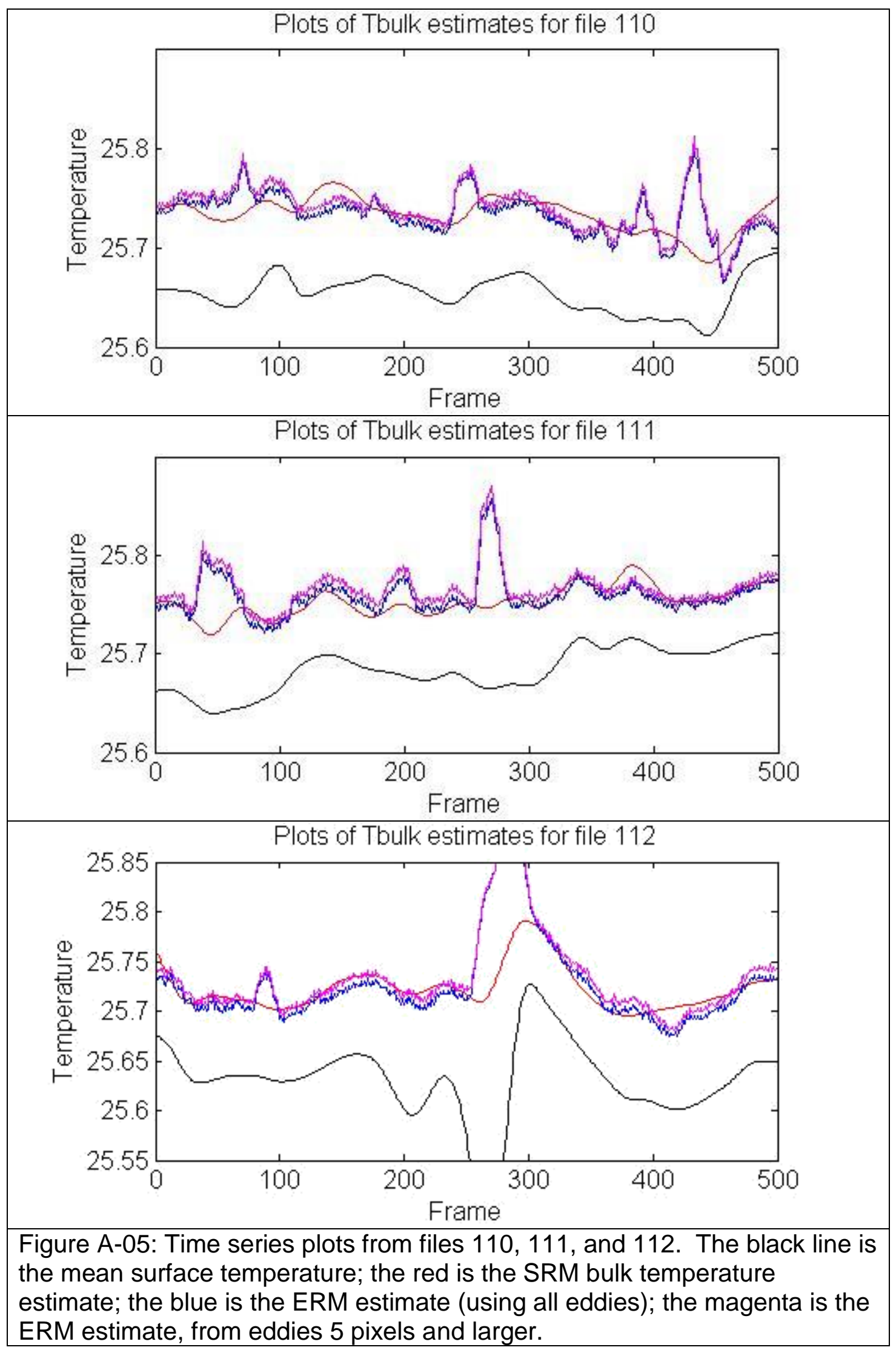




\section{Aeolotron (Wind-Wave Tank) Results}

These times series plots are the results of analysis from the wind-wave tank data. It is interesting to note the differences between these plots and the time series plots for the in situ data. The difference between the surface temperature and the estimated bulk temperature is much smaller than that for the in situ data. (For reference, the temperature scale for these plots is the same as the GasEx2001 plots $-0.3^{\circ} \mathrm{C}$ ). The validity of this observation (and the analysis) is born out by other analyses of wind-wave tank temperature profiles, which state that this condition (smaller difference between surface and bulk temperatures) is common. There are no Surface Renewal Model bulk temperature estimates for these data.

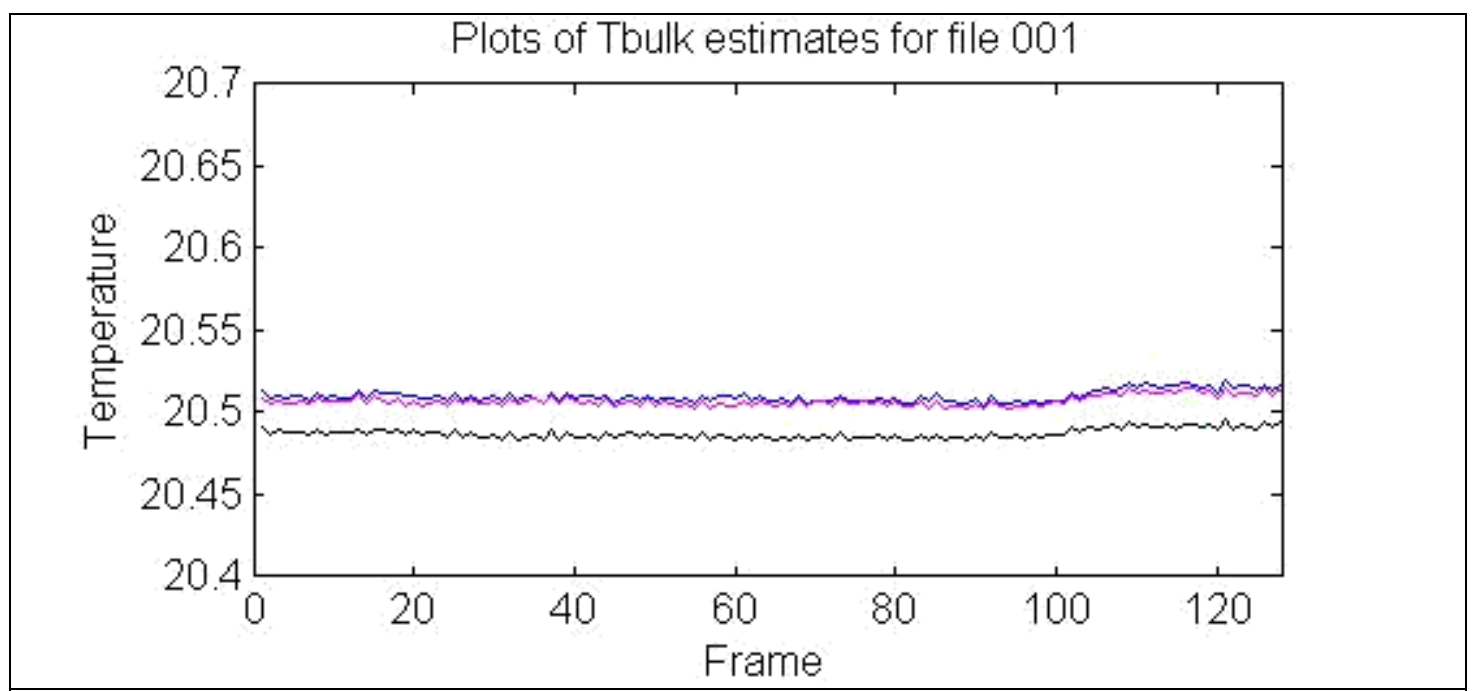

Figure A-06: Time series plots from Aeolotron file 001. The black line is the mean surface temperature; the blue is the ERM estimate (using all eddies); the magenta is the ERM estimate, from eddies 5 pixels and larger. 


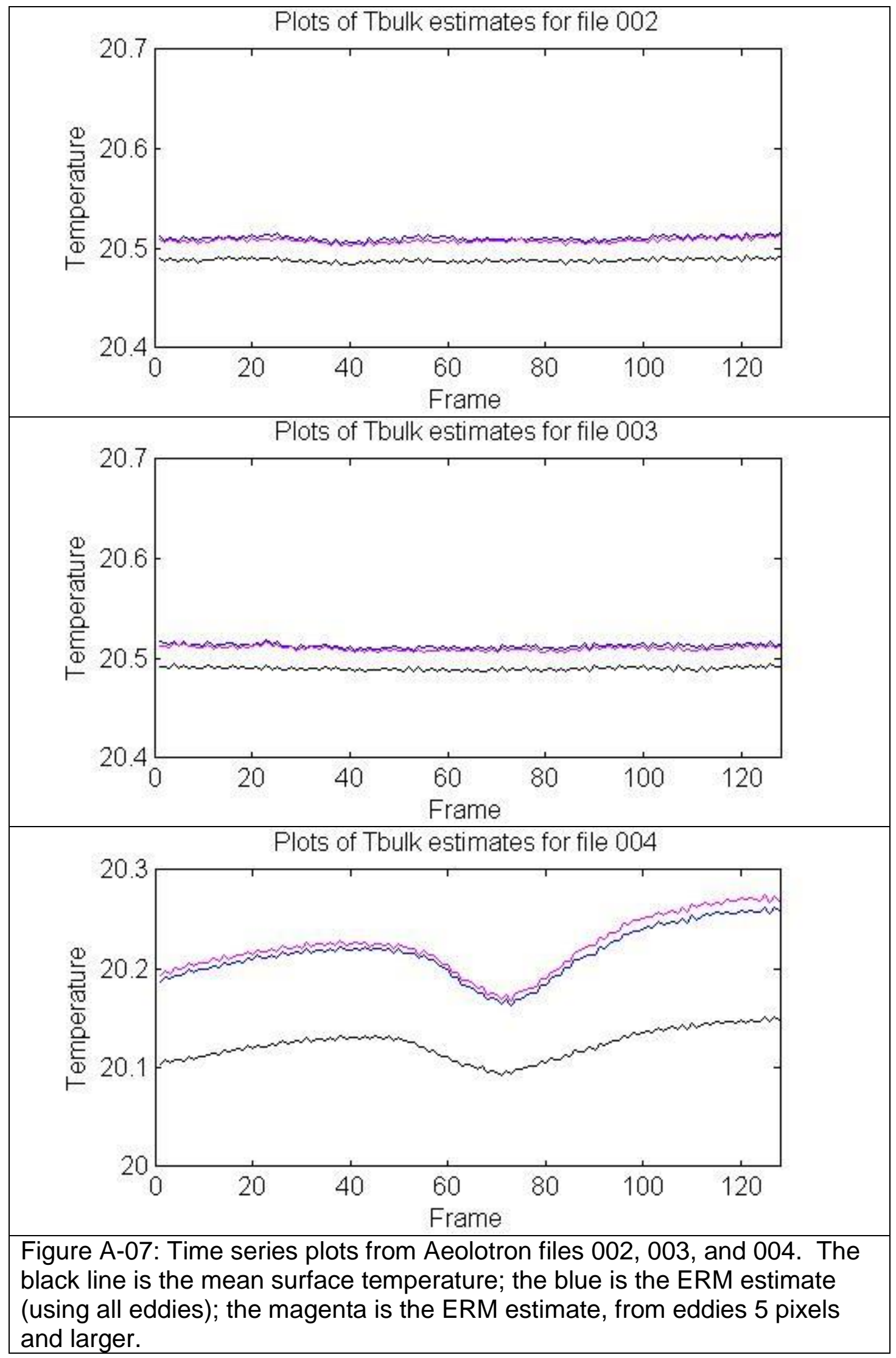




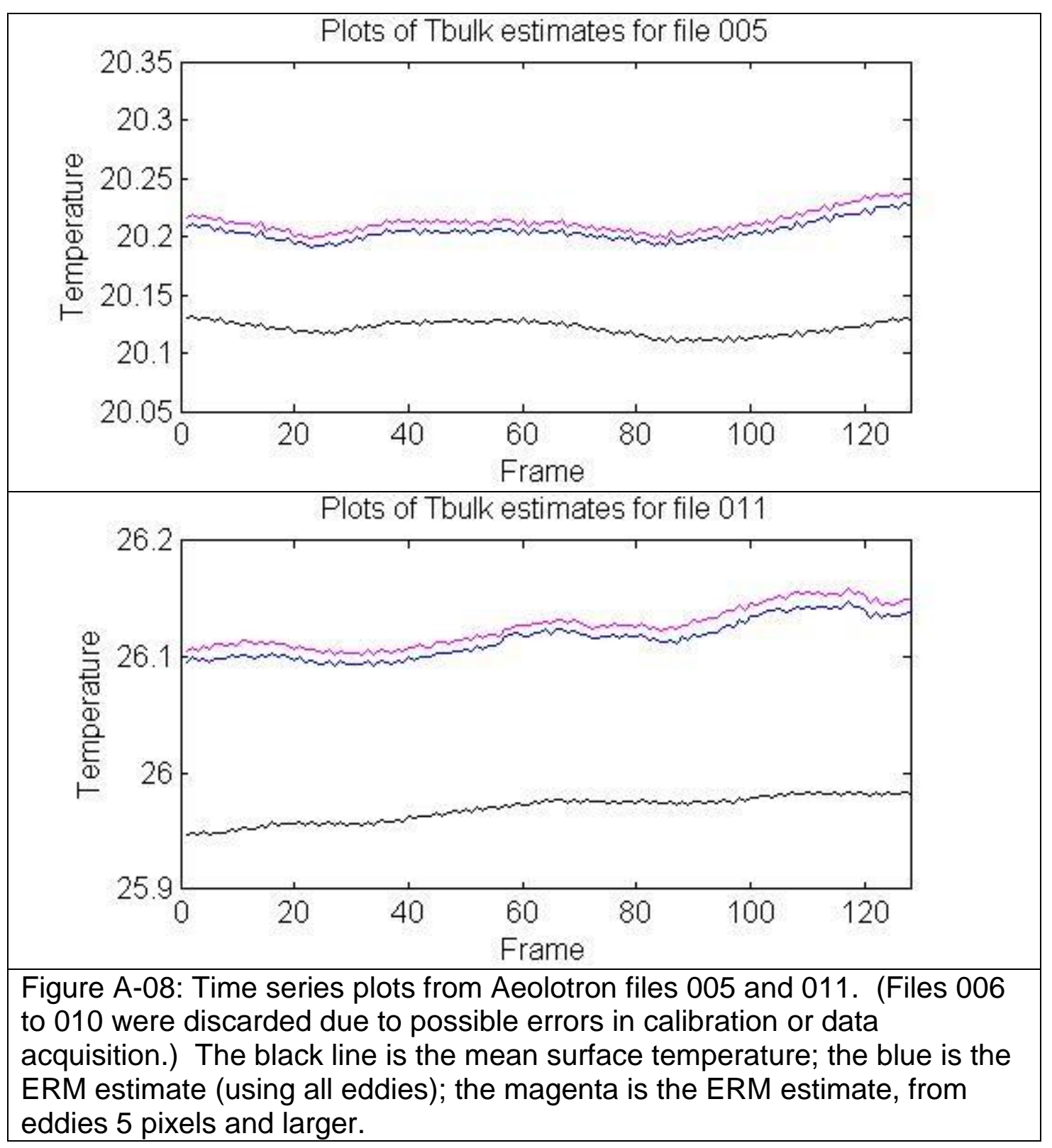




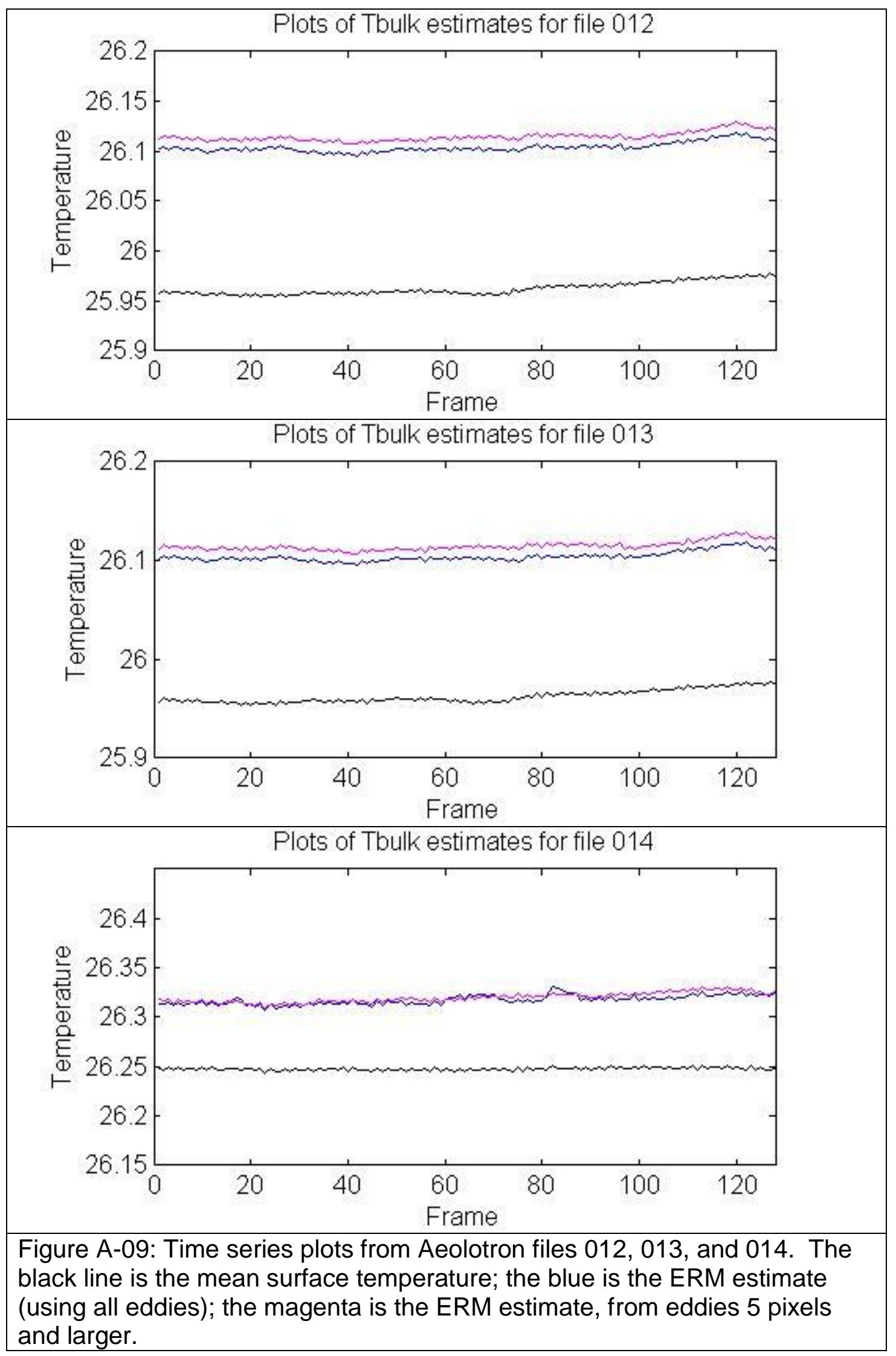




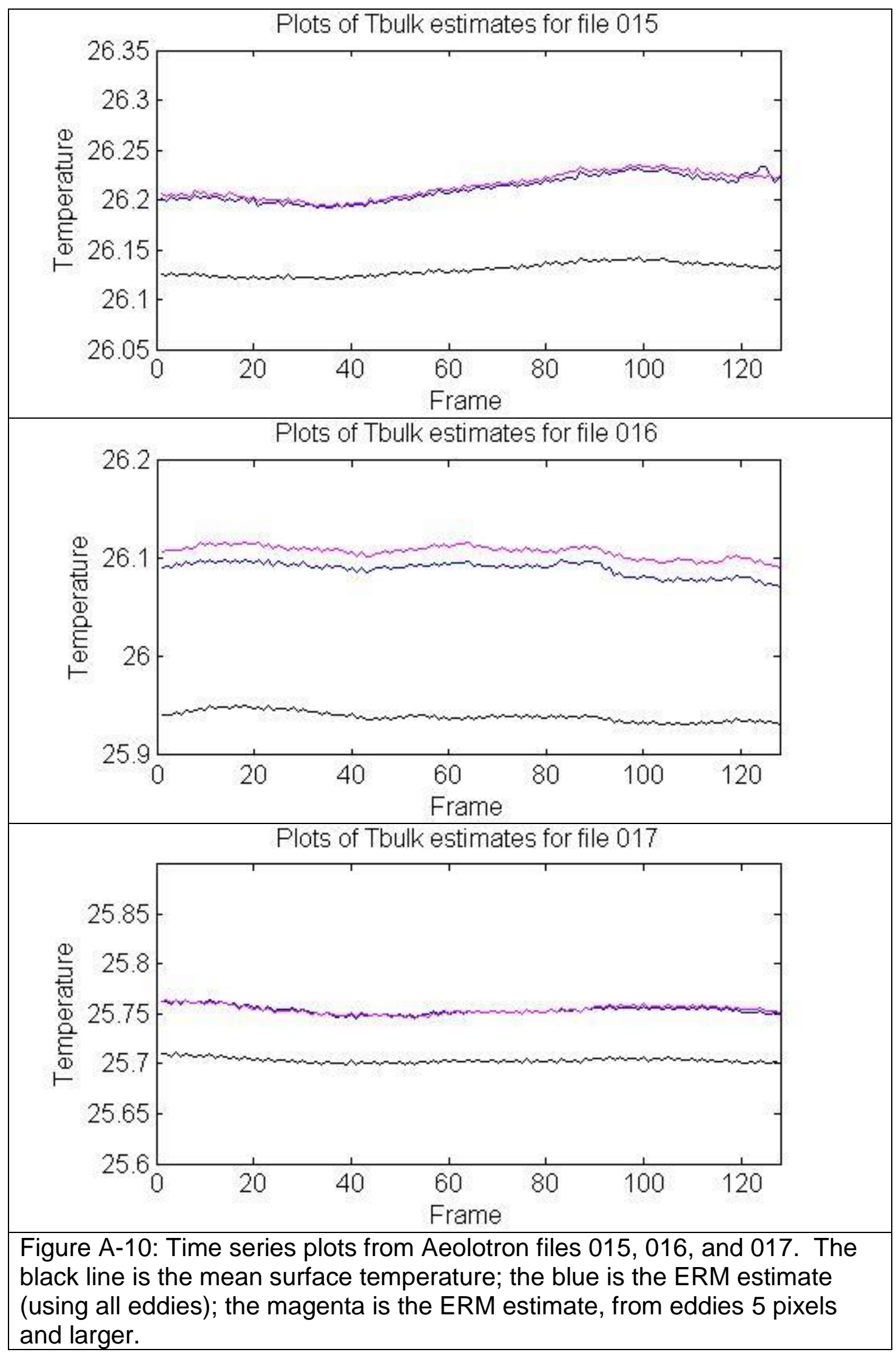




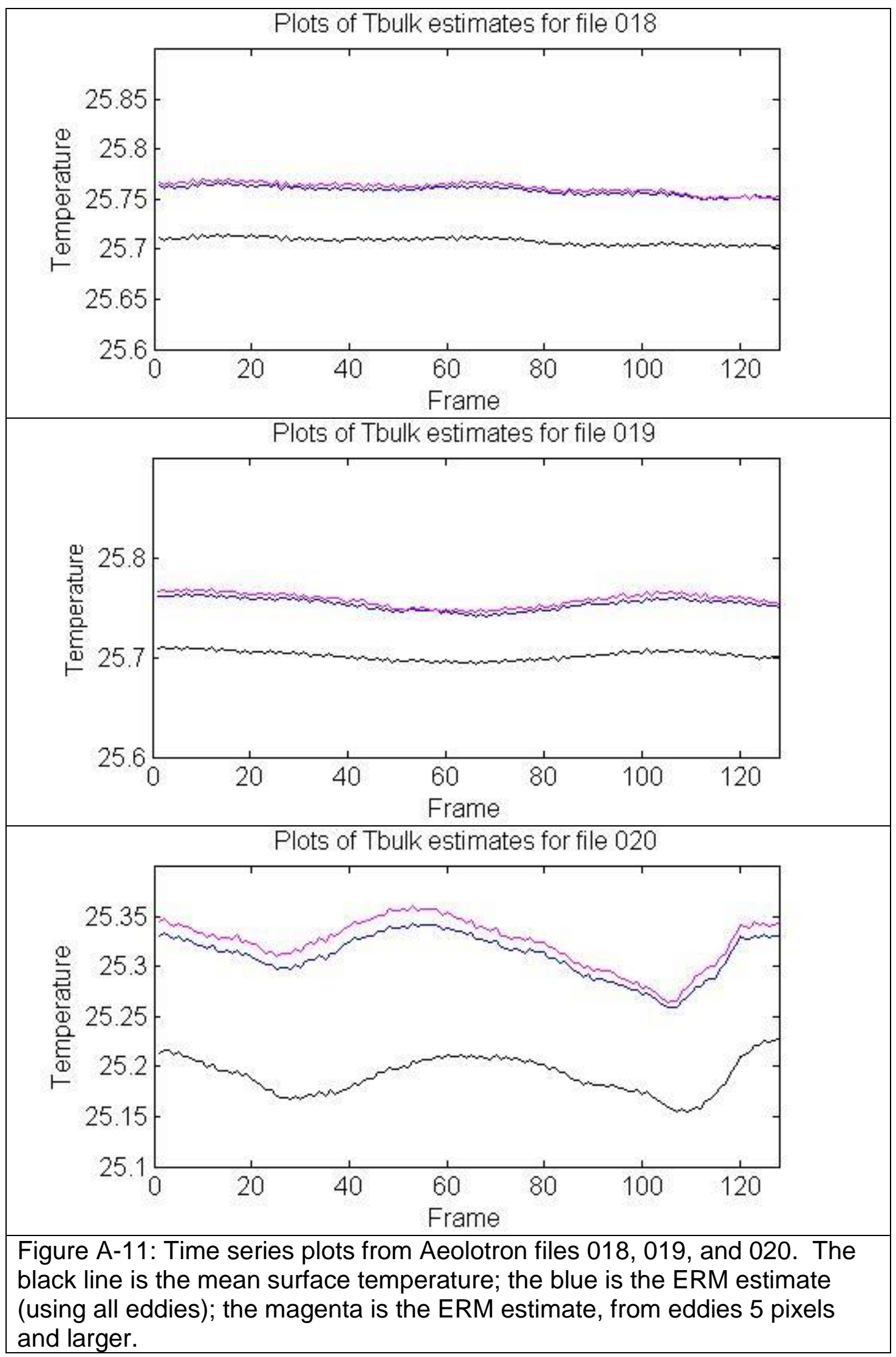



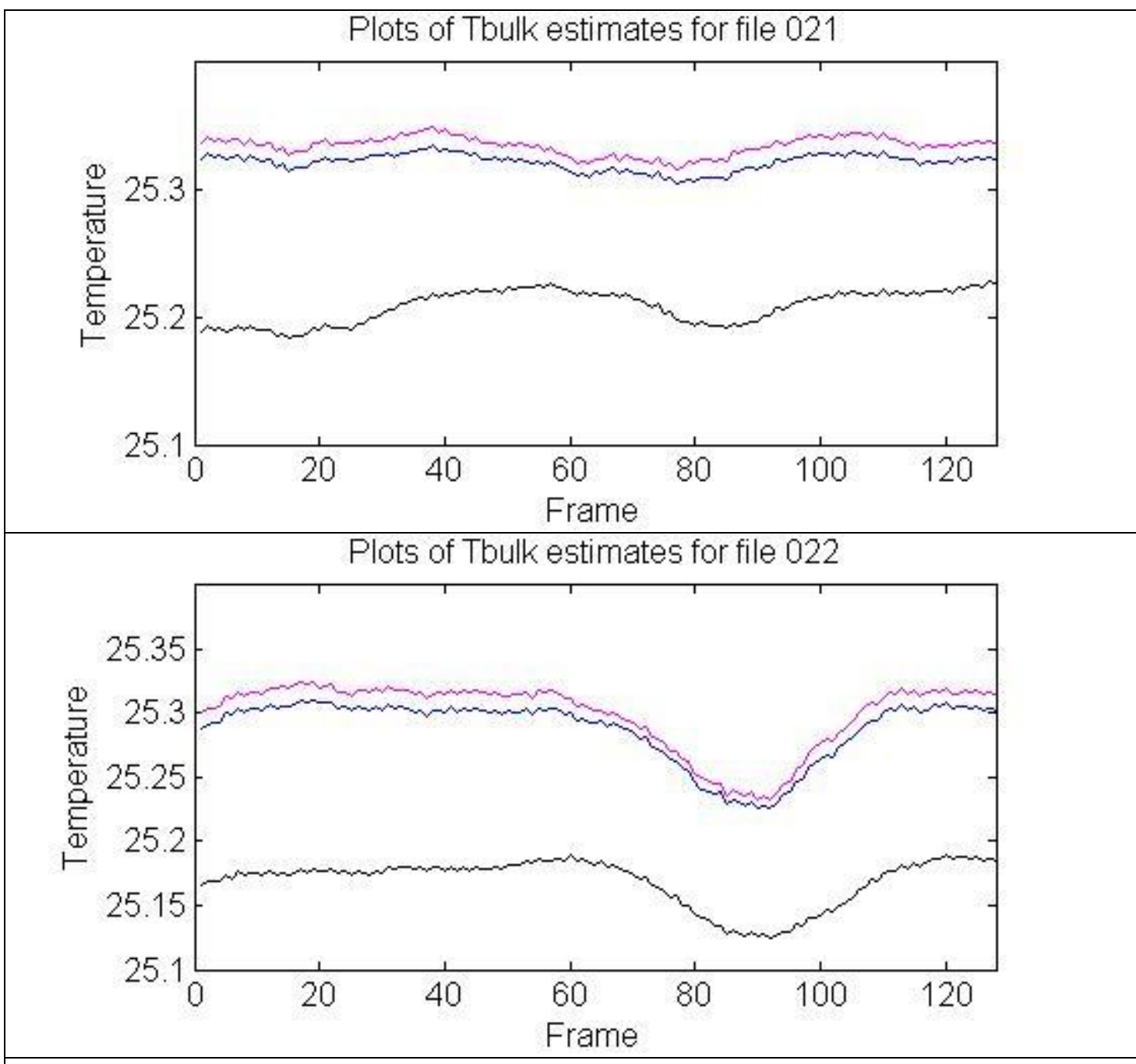

Figure A-12: Time series plots from Aeolotron files 021 and 022 . The black line is the mean surface temperature; the blue is the ERM estimate (using all eddies); the magenta is the ERM estimate, from eddies 5 pixels and larger. 


\section{CoOP1997 Time Series Results}

These time series come from analysis of data taken during the Coastal Oceans Program 1997 expedition. This data is much different from that in the previous sections. The technology used to acquire this data was older and not able to continuously take infrared images. Therefore the frames are taken not one hundredth of a second apart (as for the GasEx2001 and Aeolotron data), but rather one second apart. Also, after eight images, there is a pause of 52 seconds (which accounts for the discrete jumps every eight frames). 


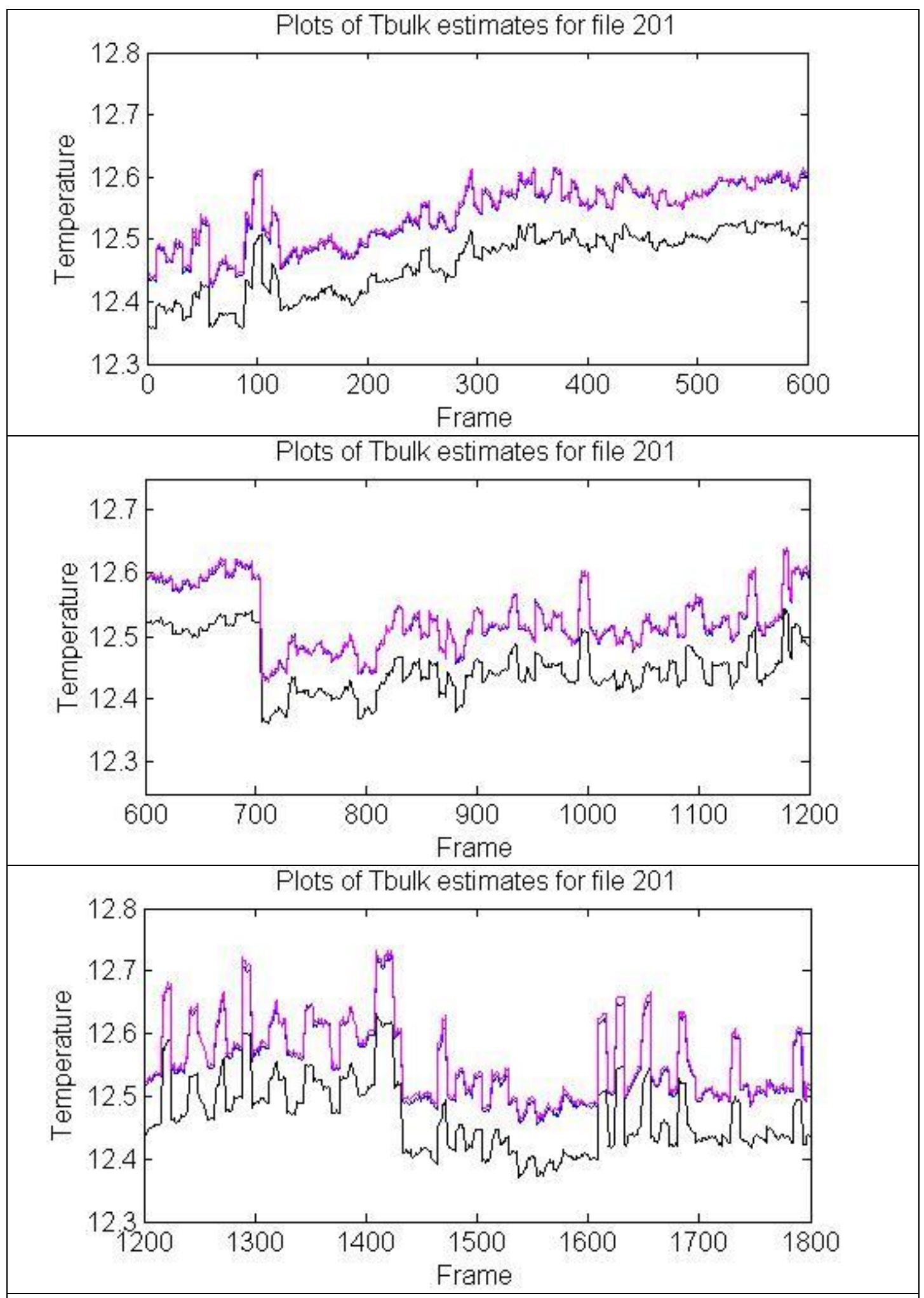

Figure A-13: Time series plots for bulk temperature estimates for Co-OP file 201. The temperature scale for this Figure is different from the other Figures in this appendix. The color scheme is the same as in the other Figures. 


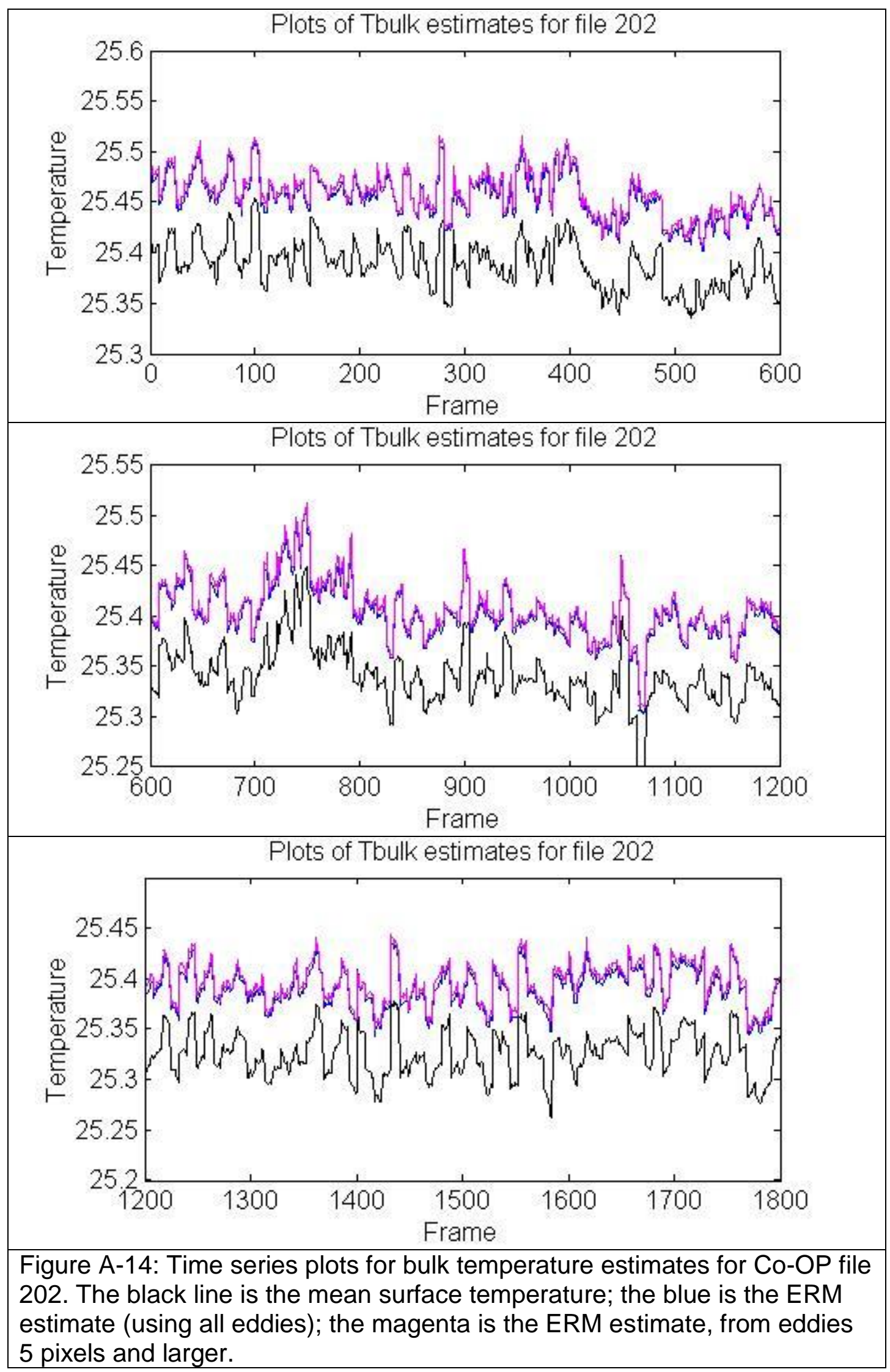




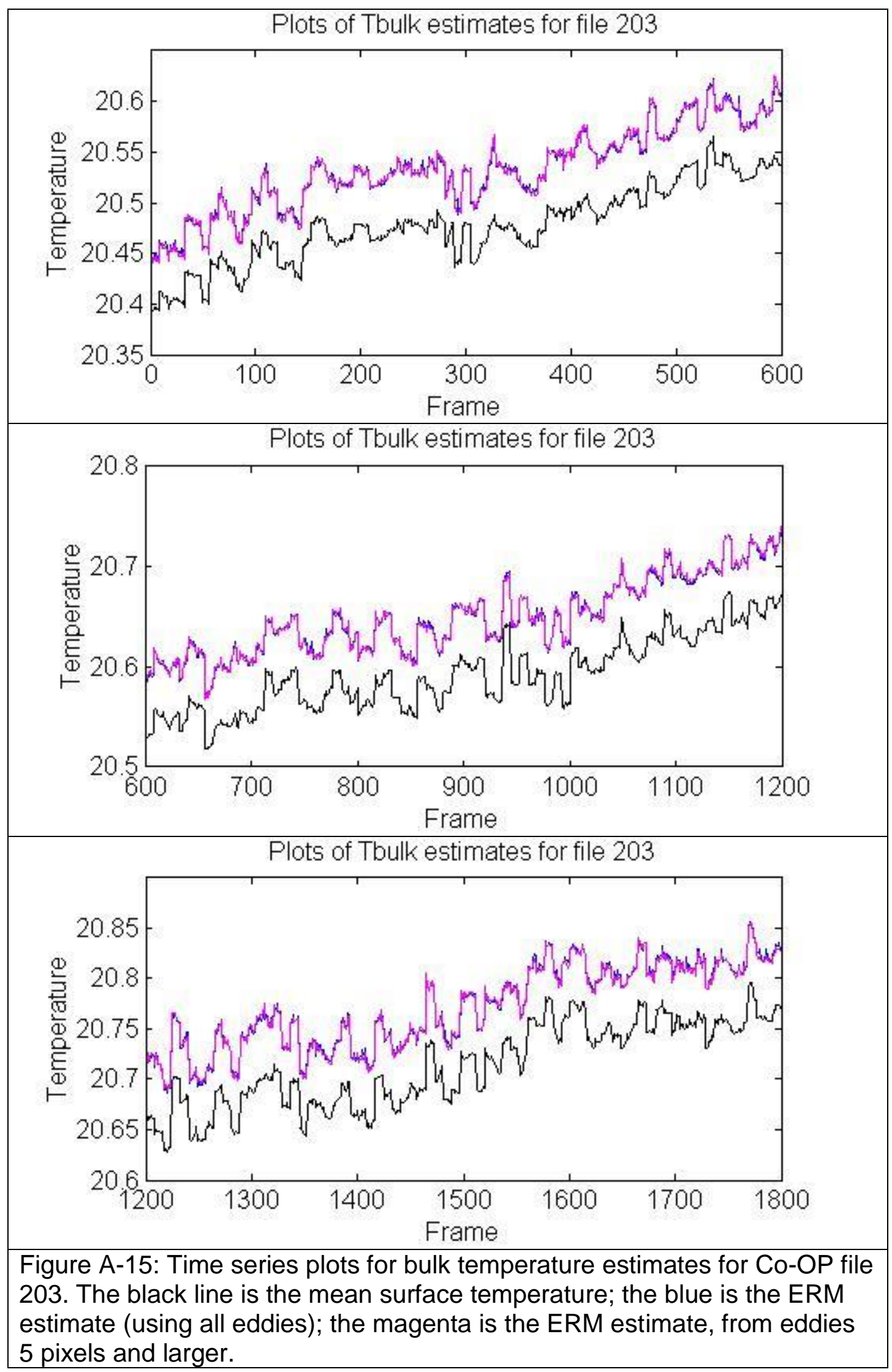




\section{Appendix C. Computational Methods and Algorithms}

The MatLab code that follows is the major computational algorithm used in these analyses. It is used within a simple automating program that reads in the appropriate files (original infrared data and eddy renewal model surface temperature curve - which was generated using code developed at University of Rhode Island's Graduate School of Oceanography by Tetsu Hara and John Wendelbo). The automating program is also designed to allow the analysis of many data files without further user interaction. Below is the core code used in the image analyses contained in this thesis; simple routines (such as generation of Figures and loading data) have been removed.

(Following MatLab formatting, comments are preceded by a '\%.')

$\%$ used to find maxima and minima along one line of data - assumed to be $\%$ perpendicular to wind, then plot a modeled curve based upon a constant $\%$ Tbulk and an intensity for each eddy. The intensity is based on the $\%$ distance from Tbulk to the mean temperature of an eddy. Then, for each $\%$ eddy, the Tbulk is found by a best fit analysis.

$\%$ this is Method 3

$\%$ using variable Tbulk \%

$\%$ and defining inten. $\%$

$\%$ based on avg temp \%

$\%$ Differs from Method $1 \%$

$\%$ by shifting curve fit\%

$\%$ and discounting mins \% 


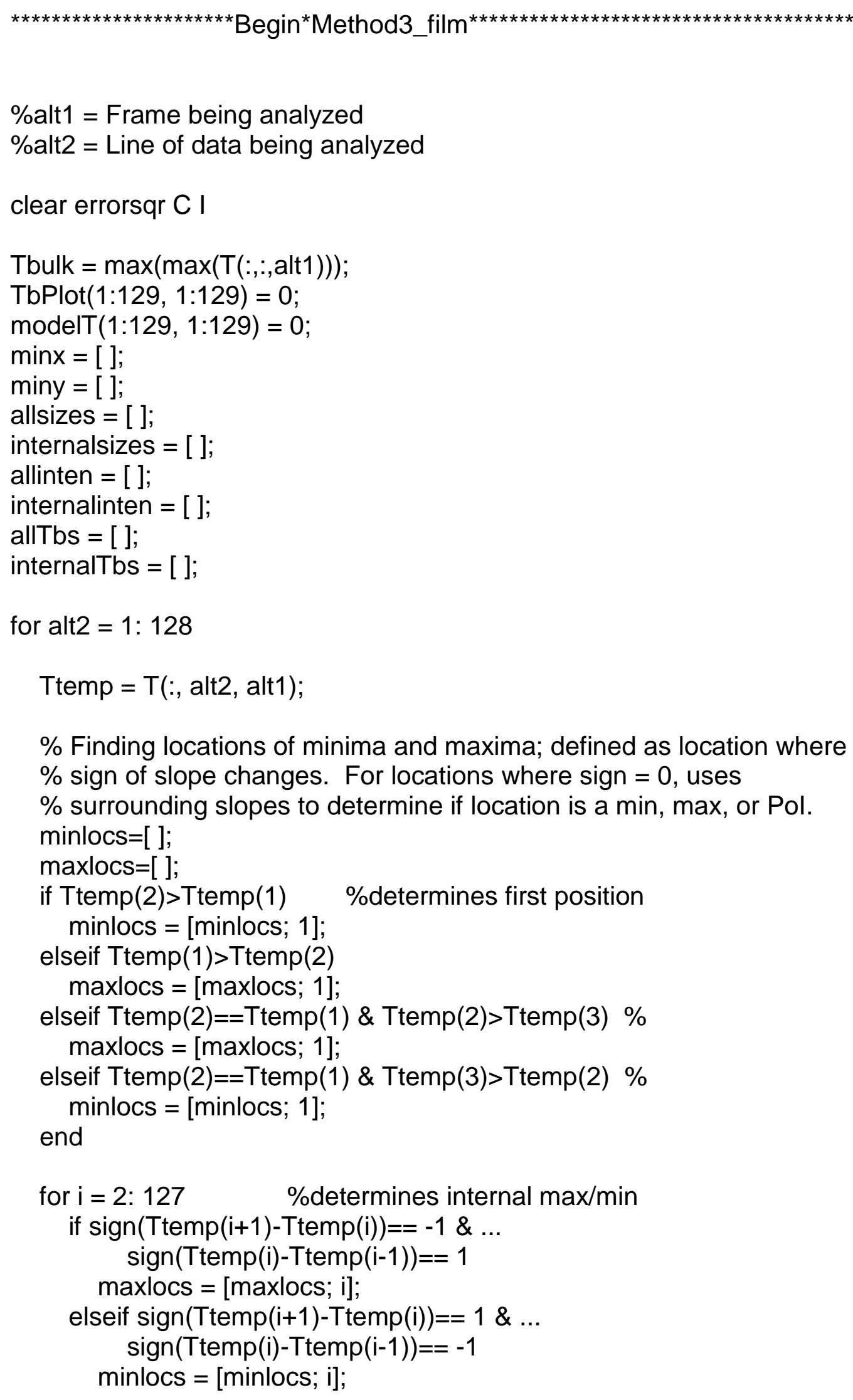




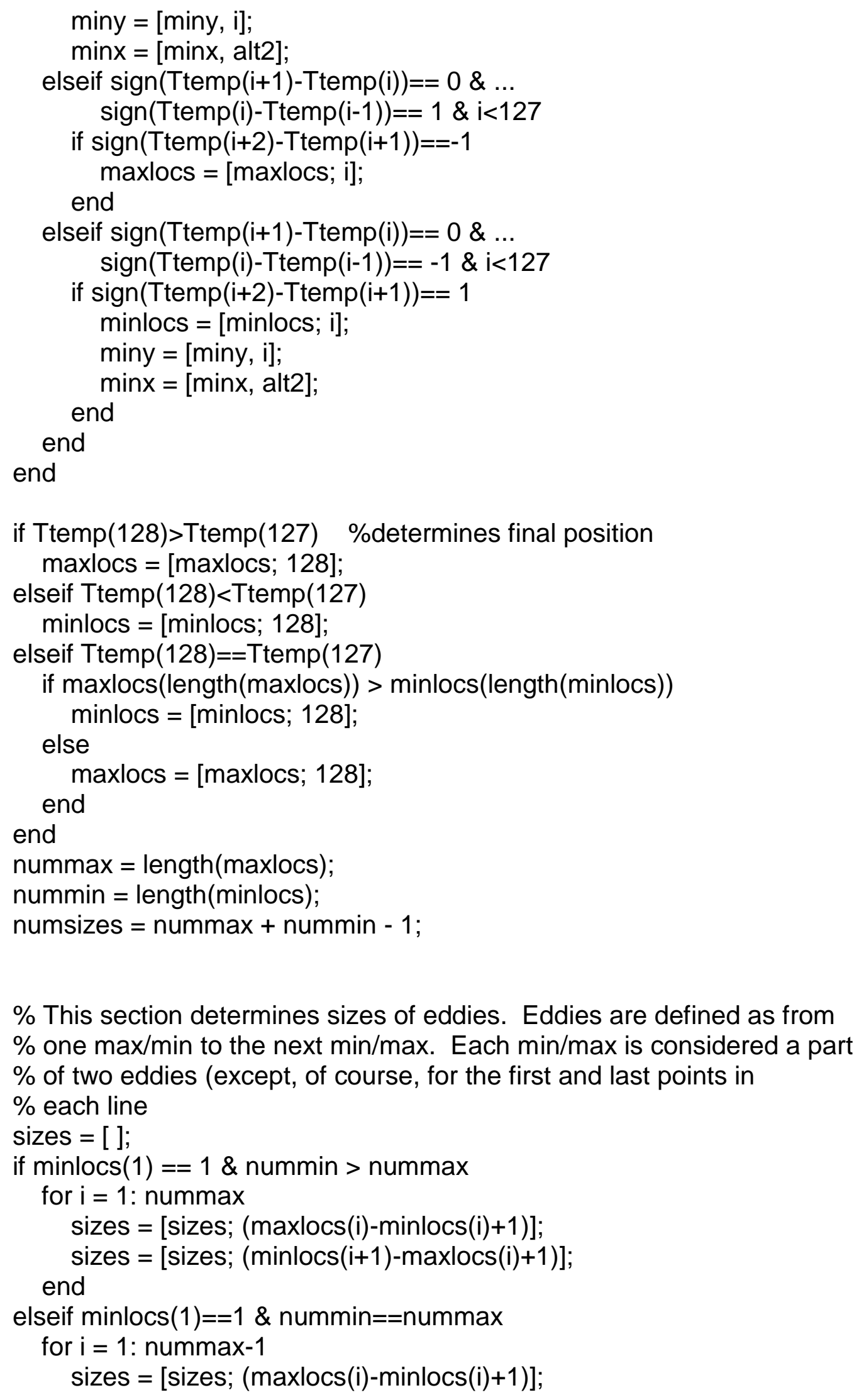

$\%$ This section determines sizes of eddies. Eddies are defined as from $\%$ one $\mathrm{max} / \mathrm{min}$ to the next $\mathrm{min} / \mathrm{max}$. Each $\mathrm{min} / \mathrm{max}$ is considered a part $\%$ of two eddies (except, of course, for the first and last points in $\%$ each line sizes $=[]$;

if $\operatorname{minlocs}(1)==1 \&$ nummin $>$ nummax 


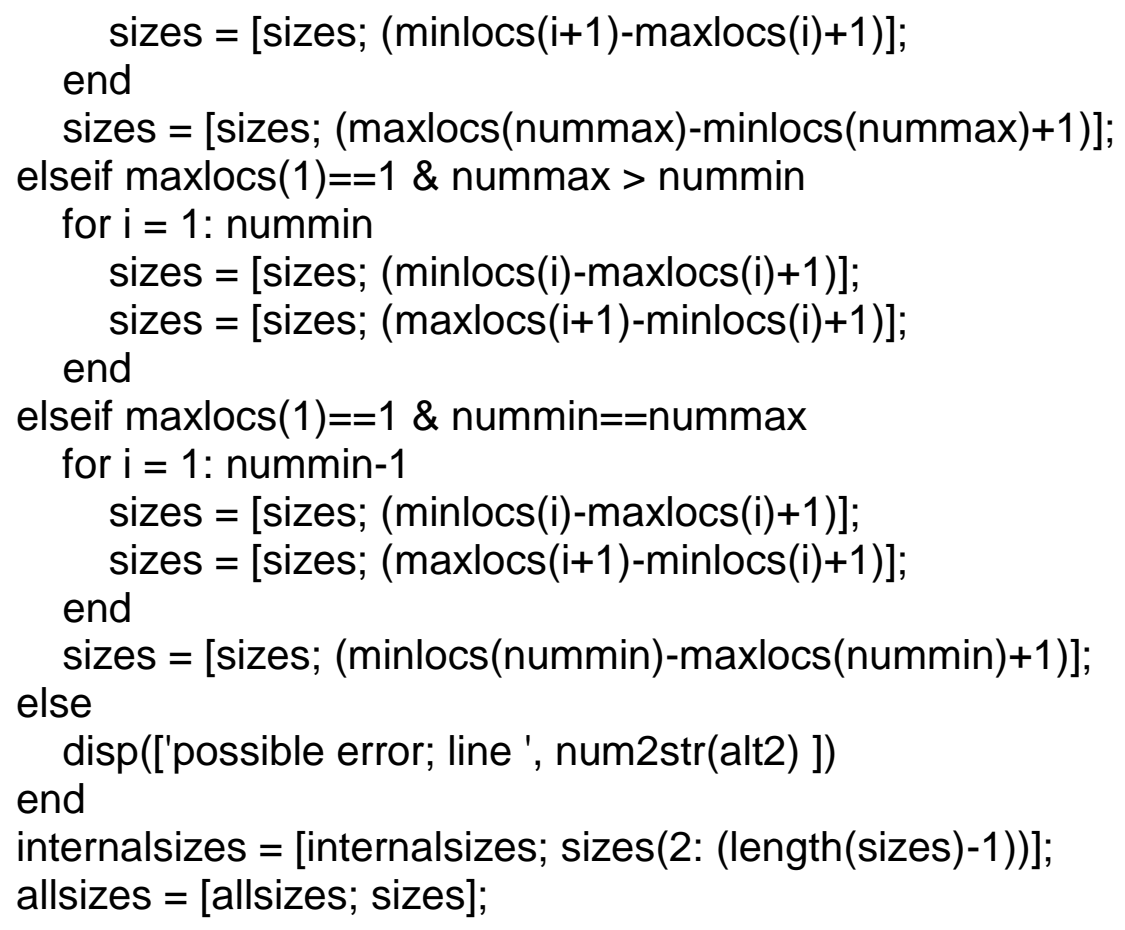

$\%$ This section finds the intensity and Tbulk for each eddy. Intensity $\%$ is defined as Tbulk-mean. In method3, the minima are used for $\%$ location, but not curve fitting. Thus, the mean of the observed curve $\%$ is defined as the average of the (sizes(i) -1) pixels, excluding the $\%$ lowest. This mean is devided by the mean of only part of the model. $\%$ The amount of the model to use is determined by dividing the length $\%$ of the model by (sizes(i)-1), assigning one block to the interior $\%$ pixels, and one half of one block to the maxima and minma. The $\%$ values for the endpoint will be averaged with those caluclated for $\%$ the next eddy.

$\operatorname{TbBF}(1$ :length(sizes $))=\mathrm{NaN}$; start $=1$; Inten $=[]$;

for $\mathrm{i}=1$ : length(sizes)

stop $=$ start + sizes $(i)-1$;

clear LowFit HighFit FirstFit TempFit TestFit clear LowError HighError FirstError TempError TestError

$\%$ finding best fit Tbulk for each curve segment $\mathrm{BF}=0 ; \%$ used in while loop UpDown = 0; \% used to find direction of search TBHigh = Tbulk + 0.005;

TBLow = Tbulk - 0.005; 


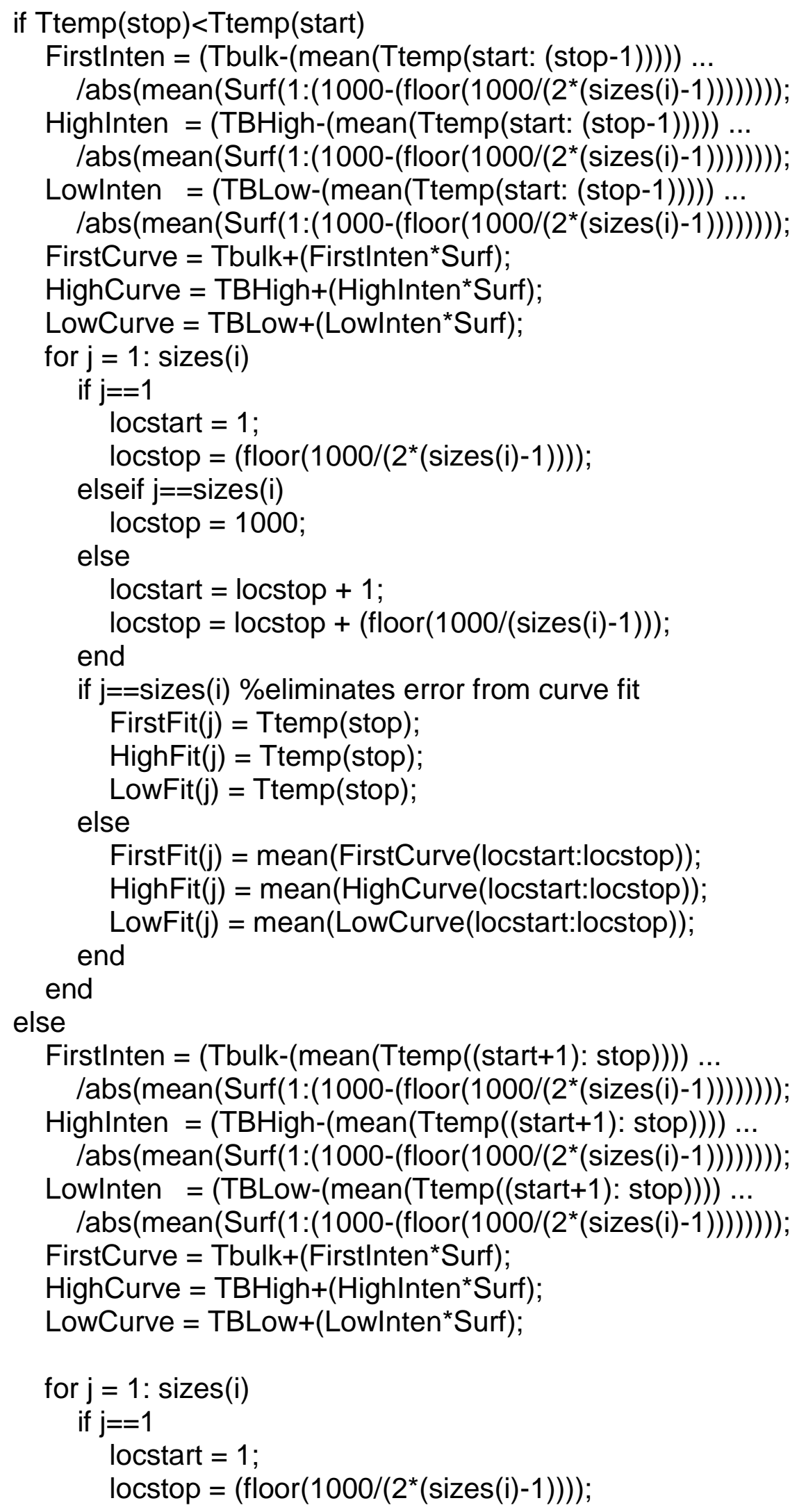




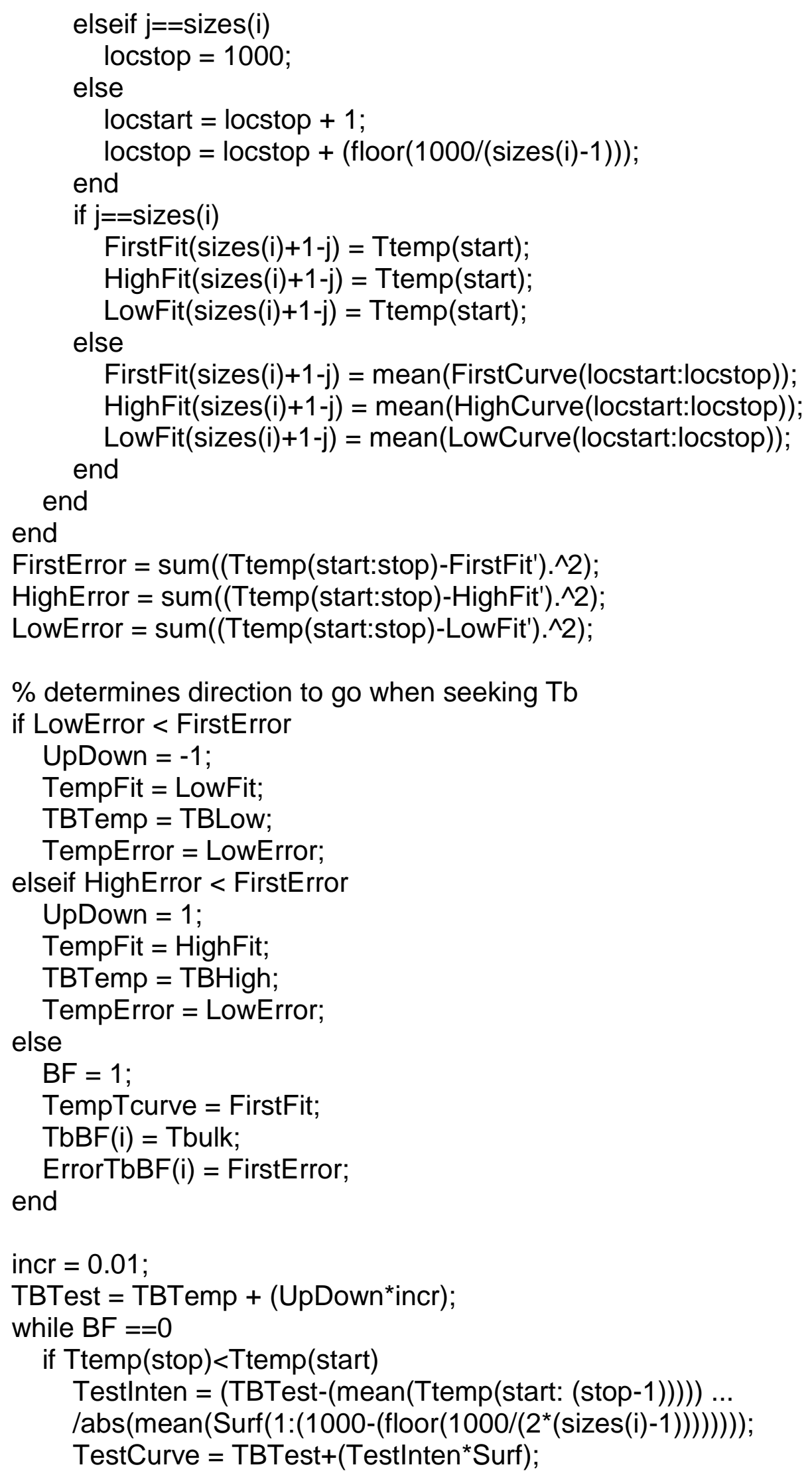




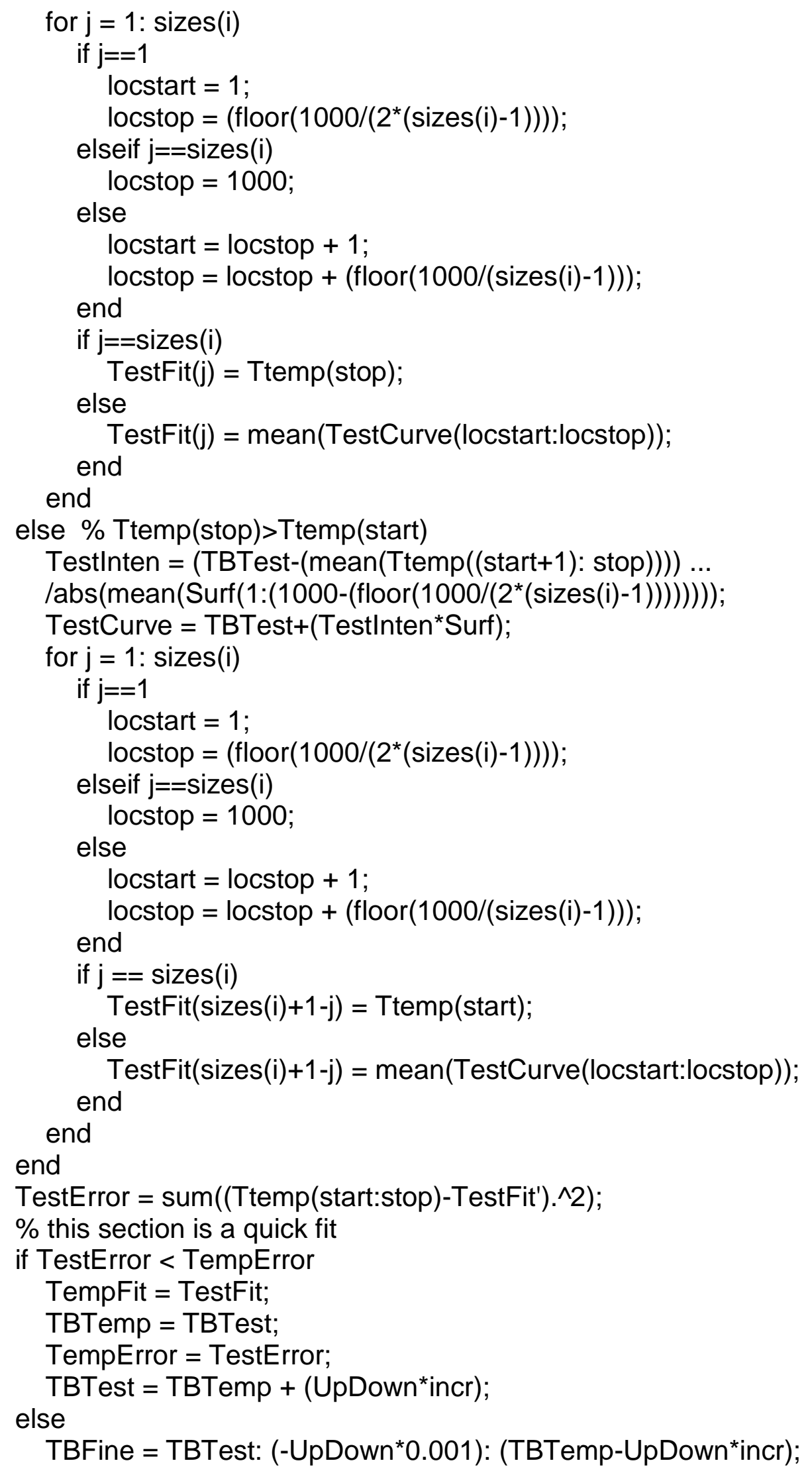

TBFine $=$ TBTest: $(-U p D o w n * 0.001):($ TBTemp-UpDown*incr); 


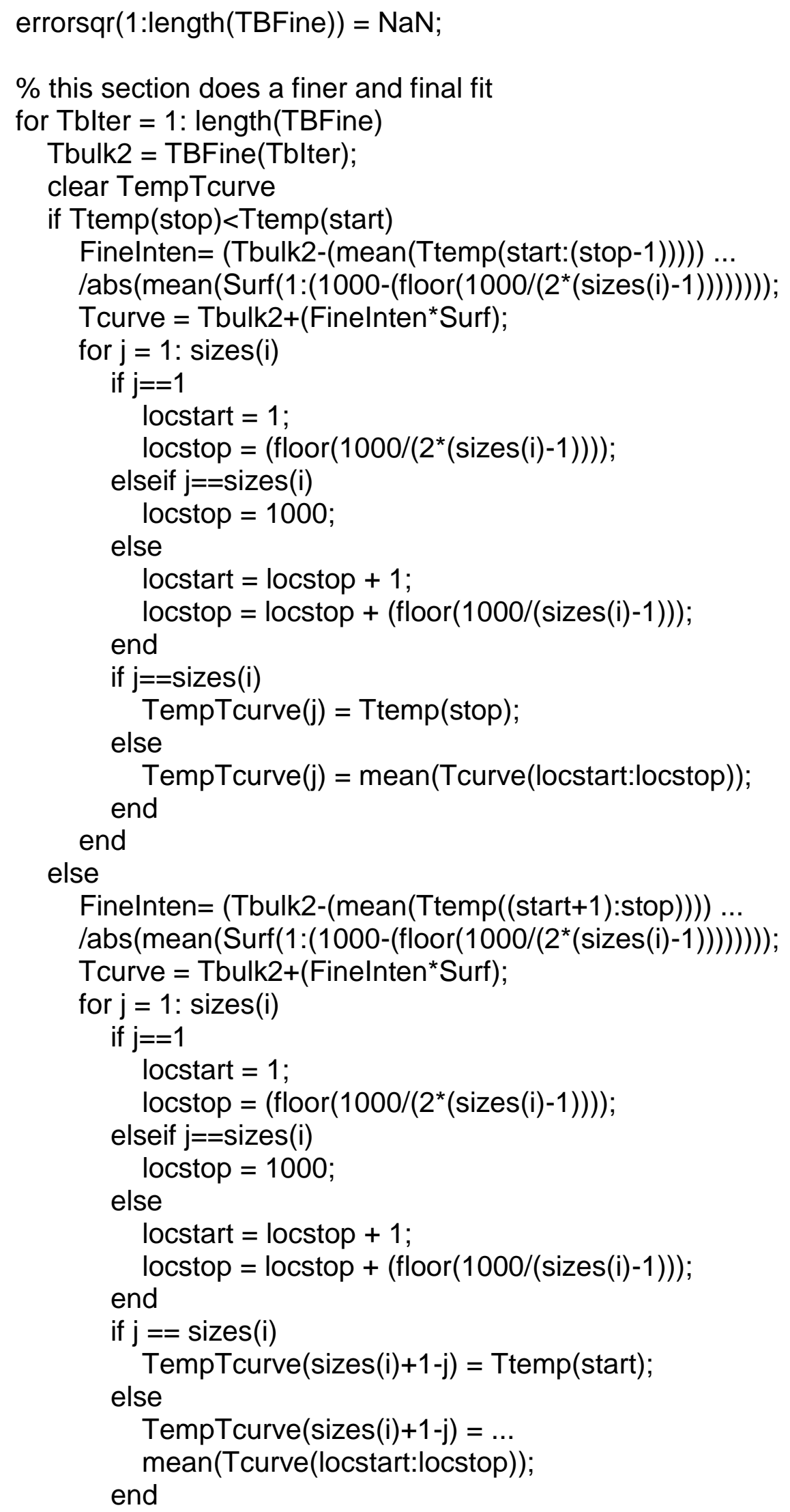




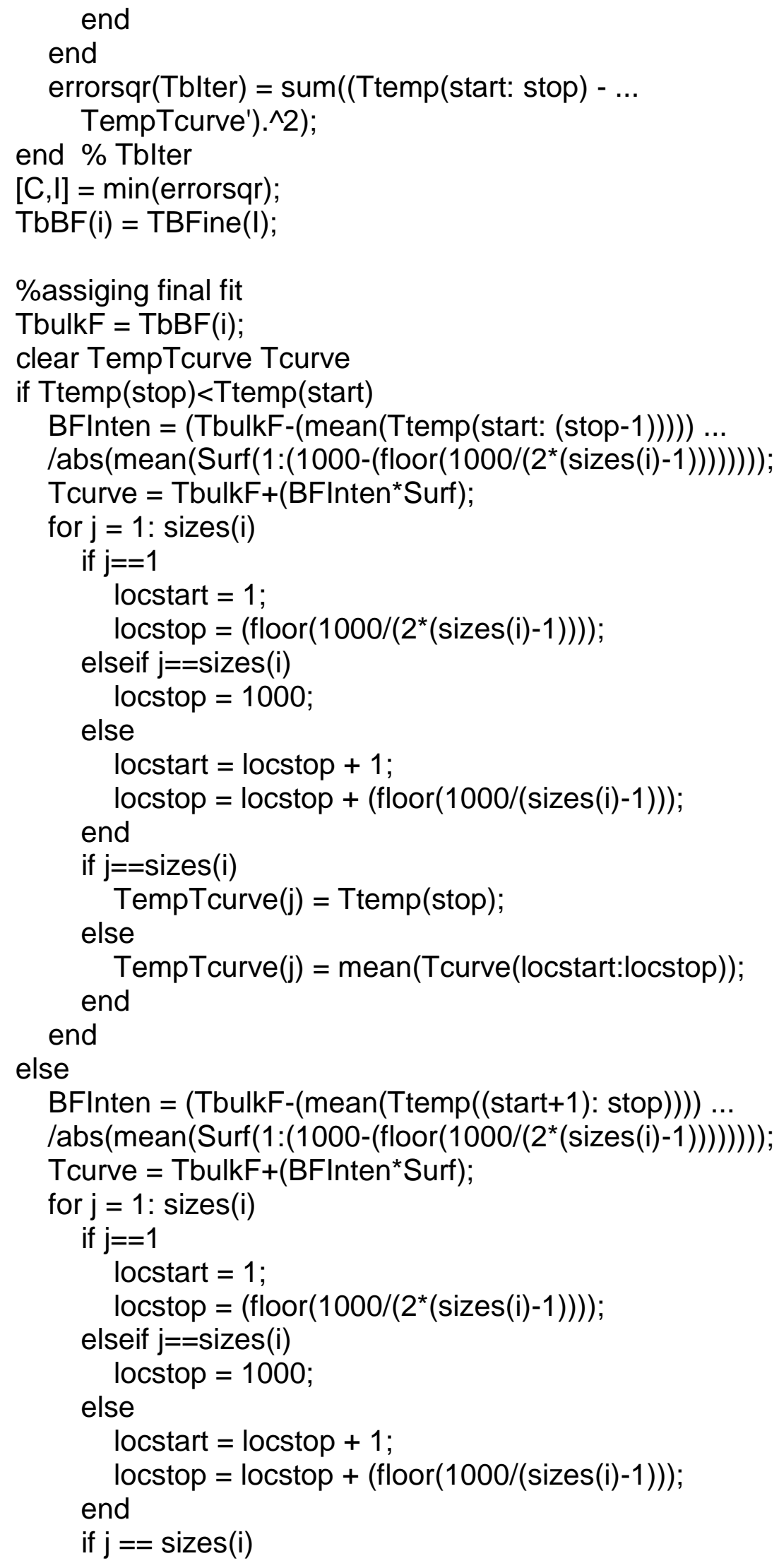




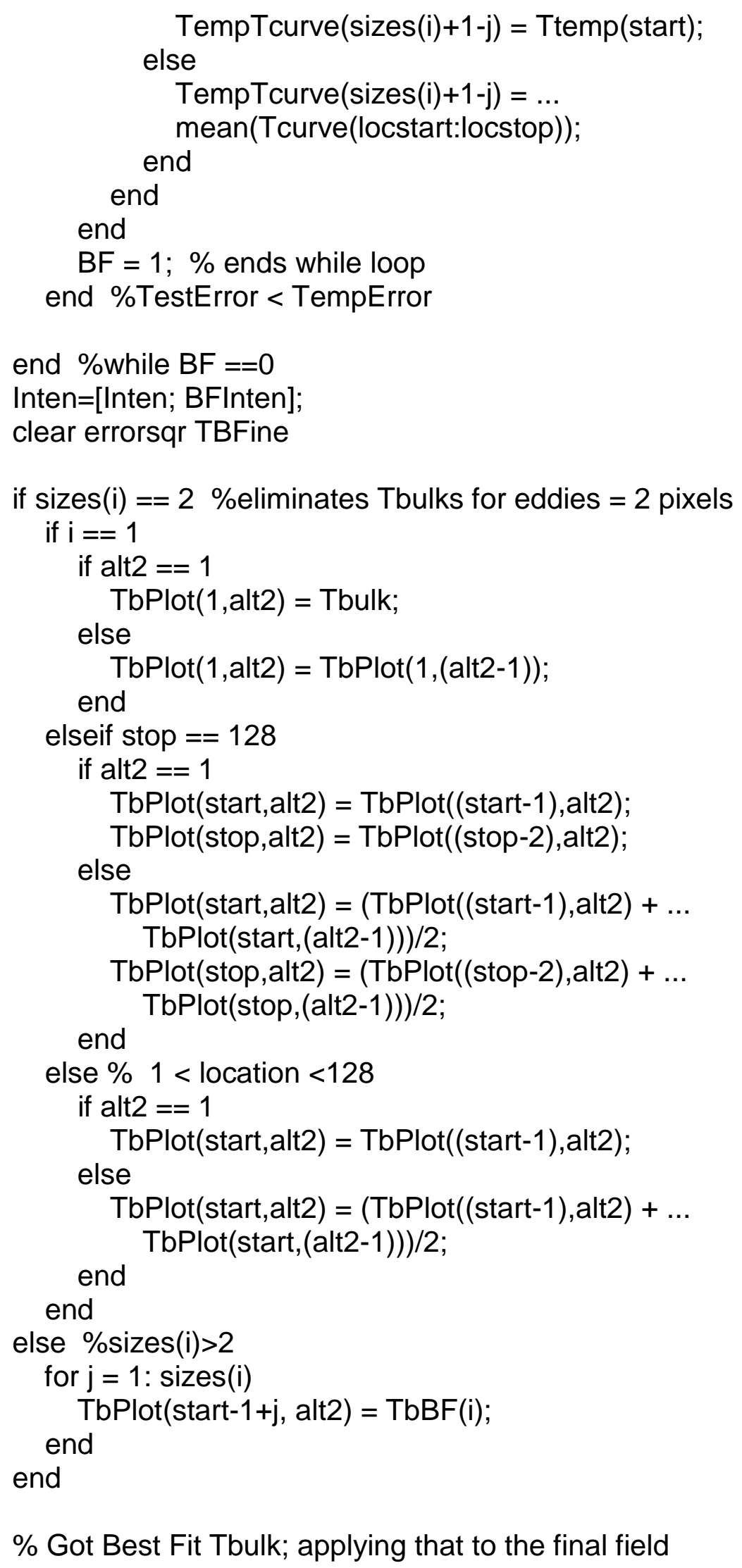

$\%$ Got Best Fit Tbulk; applying that to the final field 


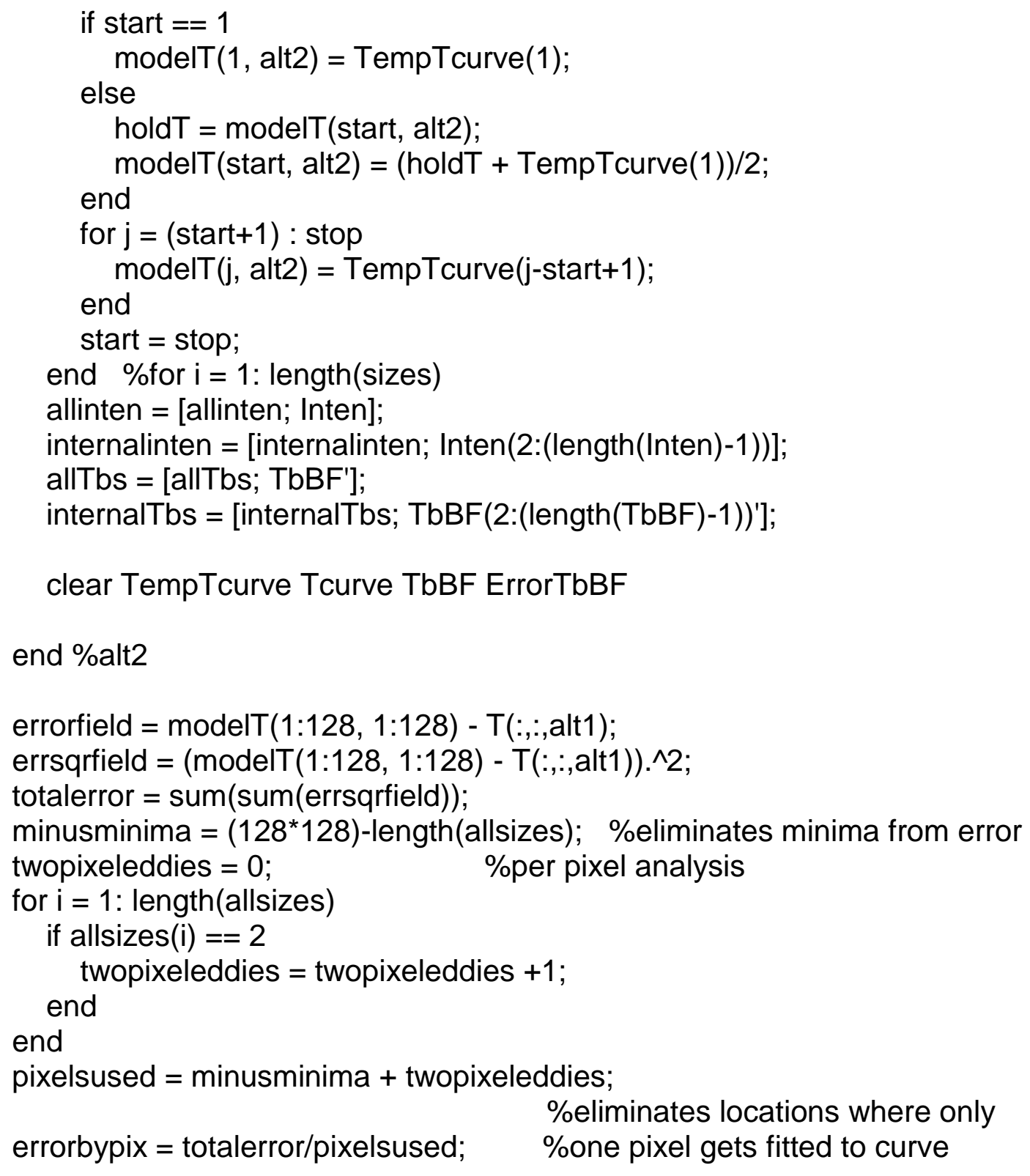




\section{Bibliography}

Atmane, M. A., W. E. Asher, and A. T. Jessup, 2004: On the use of the active infrared technique to infer heat and gas transfer velocities at the airwater free surface. Journal of Geophysical Research (C Oceans), 109

Csanady, G. T., 1990: The role of breaking wavelets in air-sea gas transfer. Journal of Geophysical Research (C Oceans), 95

Frew, N. M. and Coauthors, 2004: Air-sea gas transfer: Its dependence on wind stress, small-scale roughness, and surface films. Journal of Geophysical Research (C Oceans), 109

Fortescue, G.E. and J.R.A. Pearson, 1967: On gas absorption into a turbulent liquid. Chemical Engineering Science, 22

Garbe, C.S., 2001: Measuring Heat Exchange Processes at the Air-Water Interface from Thermographic Image Sequence Analysis. Dissertation submitted to the Combined Faculties for the Natural Science and for Mathematics of the Rupertus Carola University of Heidelberg, Germany for the degree of Doctor of Natural Sciences.

Garbe, C. S., U. Schimpf, and B. Jähne, 2004: A surface renewal model to analyze infrared image sequences of the ocean surface for the study of air-sea heat and gas exchange. Journal of Geophysical Research ( $C$ Oceans), 109

Haußecker, H., S. Reinelt, and B. Jähne, 1995: Heat as a proxy tracer for gas exchange measurements in the field: principles and technical realization. Jähne, Bernd and Monahan, Edward C. (eds. ), Air-water gas transfer: selected papers from the third international symposium on air-water gas transfer. Hanau, Germany, AEON Verlag \&amp; Studio, 1995, 405-413.

Jacobs, C., J. F. Kjeld, P. Nightingale, R. Upstill-Goddard, S. Larsen, and W. Oost, 2002: Possible errors in $\mathrm{CO}_{2}$ air-sea transfer velocity from deliberate tracer releases and eddy covariance measurements due to near-surface concentration gradients, Journal of Geophysical Research (C Oceans), 107

Jähne, B., and H. Haußecker, 1998: Air-Water Gas Exchange. Annual Review of Fluid Mechanics, 30, 443-468.

Lamont, J.C. and D.S. Scott, 1970: An Eddy Cell Model of Mass Transfer into the Surface of a Turbulent Liquid. Journal of the American Institute of Chemical Engineers, 16, v. 4. 
McGillis, W. R., J. B. Edson, J. E. Hare, and C. W. Fairall, 2001: Direct covariance air-sea $\mathrm{CO}_{2}$ fluxes. Journal of Geophysical Research (C Oceans), 106

McKenna, S.P., 2000: Free-Surface Turbulence and Air-Water Gas Exchange. Dissertation submitted to the Department of Ocean Engineering, MIT and the Department of Applied Ocean Physical and Engineering, WHOI, in partial fulfillment of the requirements for the degree of Doctor of Philosophy in Oceanographic Engineering.

Schimpf, U., C. Garbe, and B. Jähne, 2004: Investigation of transport processes across the sea surface microlayer by infrared imagery. Journal of Geophysical Research (C Oceans), 109

Soloviev, A. V. and P. Schlüssel, 1993: Parameterization of the cool skin of ocean and of the air-ocean gas transfer on the basis of modeling surface renewal. Journal of Physical Oceanography, 24

Ward, B., 2006 Near-surface ocean temperature. Journal of Geophysical Research (C Oceans), 111

Zappa, C. J., W. E. Asher, A. T. Jessup, J. Klinke, and S. R. Long, 2004: Microbreaking and the enhancement of air-water transfer velocity. Journal of Geophysical Research (C Oceans), 109 
